\title{
Robust optimization for nonlinear time-delay dynamical system of dha regulon with cost sensitivity constraint in batch culture
}

\author{
Jinlong Yuan ${ }^{\mathrm{a}, \mathrm{b}, \mathrm{c}, *}$, Xu Zhang ${ }^{\mathrm{a}}$, Chongyang Liu ${ }^{\mathrm{d}}$, Liang Chang ${ }^{\mathrm{e}, \mathrm{f}}$, Jun Xie ${ }^{\mathrm{g}}$, Enmin Feng ${ }^{\mathrm{a}}$, \\ Hongchao Yin ${ }^{\mathrm{b}}$, Zhilong $\mathrm{Xiu}^{\mathrm{c}}$ \\ ${ }^{a}$ School of Mathematical Sciences, Dalian University of Technology, Dalian 116024, Liaoning, PR China \\ ${ }^{b}$ School of Energy and Power Engineering, Dalian University of Technology, Dalian 116024, Liaoning, PR China \\ ${ }^{c}$ School of Life Science and Biotechnology, Dalian University of Technology, Dalian 116024, Liaoning, PR China \\ ${ }^{d}$ School of Mathematics and Information Science, Shandong Institute of Business and Technology, Yantai 264005, Shandong, PR China \\ ${ }^{e}$ Faculty of management and Economics, Dalian University of Technology, Dalian 116024, Liaoning, PR China \\ ${ }^{f}$ School of Physics and Optoelectronic Technology, Dalian University of Technology, Dalian 116024, Liaoning, PR China \\ ${ }^{g}$ Teaching and Research Office of Mathematics, Department of Basics, PLA Dalian Naval Academy, Dalian 116018, Liaoning, PR China
}

\begin{abstract}
Time-delay dynamical systems, which depend on both the current state of the system and the state at delayed times, have been an active area of research in many real-world applications. In this paper, we consider a nonlinear time-delay dynamical system of $d h a-$ regulon with unknown time-delays in batch culture of glycerol bioconversion to 1,3-propanediol induced by Klebsiella pneumonia. Some important properties and strong positive invariance are discussed. Because of the difficulty in accurately measuring the concentrations of intracellular substances and the absence of equilibrium points for the time-delay system, a quantitative biological robustness for the concentrations of intracellular substances is defined by penalizing a weighted sum of the expectation and variance of the relative deviation between system outputs before and after time-delays are perturbed. Our goal is to determine optimal values of time-delays. To this end, we formulate an optimization problem in which the time-delays are decision variables and the cost function is to minimize the biological robustness. This optimization problem is subject to the time-delay system, parameter constraints, continuous state inequality constraints for ensuring that the concentrations of extracellular and intracellular substances lie within specified limits, a quality constraint to reflect operational requirements and a cost sensitivity constraint for ensuring that an acceptable level of the system performance is achieved. It is approximated as a sequence of nonlinear programming subproblems through the application of constraint transcription and local smoothing approximation techniques. Due to the highly complex nature of this optimization problem, the computational cost is high. Thus, a parallel algorithm is proposed to solve these nonlinear programming sub-problems based on the filled function method. Finally, it is observed that the obtained optimal estimates for time-delays are highly satisfactory via numerical simulations.
\end{abstract}

\section{(c) 2015 Published by Elsevier Ltd.}

Keywords: Nonlinear time-delay system, Biological robustness, Cost sensitivity constraint, Parallel optimization

\section{Introduction}

The microbial conversion of glycerol induced by Klebsiella pneumonia (K. pneumonia) to 1,3-propanediol (1,3PD) is particularly attractive to industry since it is environmentally safe and has renewable feedstock [1, 2, 3, 4, 5].

\footnotetext{
${ }^{*}$ Corresponding author

Email addresses: yuanjinlong0613@163.com (Jinlong Yuan), hcyin@dlut.edu.cn (Hongchao Yin)
} 
The fermentation process can be divided into three types: batch culture (all substrate is added to the fermentor at the beginning of the reaction); continuous culture (substrate is added continuously during the reaction); and fed-batch cultures (the process switches between batch and continuous modes) [6,7]. There are three reasons for the necessity of studying batch culture [8]. First, batch culture lays an experimental foundation for fed-batch or continuous culture. In other words, batch culture is a part of fed-batch or continuous cultures. Second, batch culture compared to fedbatch or continuous culture can obtain the highest conversion rates because it can express the process of the excess metabolism for the bioconversion of glycerol, while other cultures fail to achieve this. Finally, the waste water, in which the concentration of glycerol is relatively low for the lipidic chemical or biodiesel company, is appropriate to the process of batch fermentation. In the laboratory, it is impractical to carry out a sufficient number of experiments so as to obtain high production concentration and molar yield 1,3-PD to glycerol. Thus, the need for the mathematical model or system, which describes this microbial process, is evident. In [9], a substrate-sufficient kinetic system is presented to describe substrate (biomass, glycerol) consumption and extracellular substances (1,3-PD, acetate and ethanol) formation in batch culture. A nonlinear dynamical system involving concentration changes of three intracellular substances and two key enzymes is proposed in [10]. Based on these mathematical models, batch culture has become a hot research topic, including suboptimal control problems [11], robust optimal control problems [12], multi-stage system [13, 14, 15], sensitivity analysis [16], robust identification problems [17], pathway identification problems [18], stochastic model [19] joint estimation [20] and hybrid system [21].

However, for all the papers mentioned above, the global regulation of gene expression is not taken into consideration. There are four typical key enzymes for the glycerol metabolism process, including 1,3-PD oxydoreductase (PDOR), glycerol dehydratase (GDHt), glycerol dehydrogenase (GDH) and dihydroxyacetone kinase I (DHAK I). These enzymes are coordinately expressed and induced by glycerol or dihydroxyacetone (DHA). The genes, which are called the dha regulon $[22,23]$, are organized as a cluster on the chromosome for these four enzymes. The dha regulon can regulate the four key enzymes. The expression of the dha regulon will be repressed by the intermediate 3-HPA [24]. These facts make the dha regulon the key factor in the process of glycerol metabolism. In [25], a fourteen-dimensional nonlinear dynamical system was established to describe the batch culture including enzymecatalytic kinetics and genetic regulation. More recently in [26], a pathway identification for a nonlinear hybrid system is studied on the basis of the results in [25].

Unfortunately, in [26], there is one limitation: time-delay is not considered. Time-delay systems are frequently encountered in a multitude of real-world applications over the past decade, for example, see [27, 28, 29, 30, 31, $32,33,34,35,36]$ and the references cited therein. Several reasons may be responsible for the occurrence of the delays in the fermentation process: (i) a cell has to undergo some change or growth process for which it needs some time before it reacts with others; (ii) the substrate and the products have to be transported across the cell membrane requiring a certain amount of time for transport; (iii) sometimes, either because of lack of knowledge or in order to reduce complexity it is appropriate to omit a number of intermediate steps in the reaction system for which the processing time is not negligible and has to be implemented as a delay [37]. Thus, time-delays have to be incorporated into mathematical models formulating the fermentation process. In many systems, however, the time-delays are not known exactly [38]. Hence, time-delay estimation is one of the key issues in the study of time-delay systems [39]. A parameter identification problem for a nonlinear time-delay system behaves as follows: at each time $t$, the system's instantaneous rate of change depends not only on its current state, but also on its state at times $t-\tau_{i}, i=1, \ldots, m$, where each $\tau_{i}$ is a so-called state-delay [40].

Considering the difficulties in measuring the concentrations of intracellular substances, biological robustness is a popular concept to judge the reliability of numerical solution for the concentrations of intracellular substances [41]. Perc and Marhl [42, 43] defined the biological robustness, which is usually evaluated by the sensitivity analysis and frequency. Stelling [44] deemed that robustness is a fundamental feature of living systems where its relationship with evolution-trade-offs among robustness, fragility, resource demands, and performance-provides a possible framework for how biological systems have evolved and been organized. Kitano [45, 46] argued that biological robustness is a property that allows a system to maintain its functions despite external and internal perturbations. With this motivation, we quantitatively defined the biological robustness of batch culture in our previous work [26], which has one limitation: such definition only considers the expectation of the relative deviation between system outputs before and after time-delays are perturbed. One of our contributions in the paper overcomes this limitation.

Generally, a nonlinear dynamic system is an idealized description of the actual behavior of a biological or engineering system [47]. During the life span of the system, the values of some of its coefficients may change [48]. 
For an optimization problem governed by a nonlinear dynamical system, the optimal cost function obtained is under the assumption that the coefficients of the dynamical system are fixed [49]. Given some of these system coefficients are subject to variation, the sensitivity of the variation of these coefficients should be taken into consideration [50]. However, in microbial fermentation, there are few papers dedicated to this important issue.

In the paper, in batch culture of glycerol bioconversion to 1,3-PD induced by K. pneumonia, we consider a nonlinear time-delay dynamical system of dha-regulon with unknown time-delays. Some important properties and strong positive invariance are discussed. Because of the difficulty in accurately measuring the concentrations of intracellular substances and the absence of equilibrium points for the time-delay system, a quantitative biological robustness for the intracellular substances concentrations is defined by penalizing a weighted sum of the expectation and variance of the relative deviation between system outputs before and after time-delays are perturbed. Our goal is to determine optimal values of time-delays. To do this, we formulate an optimization problem subject to the time-delay system, parameter constraints, continuous state inequality constraints for ensuring that the concentrations of extracellular and intracellular substance lie within specified limits, a quality constraint for ensuring that an acceptable level of the time-delay system better fits given experimental data and a cost sensitivity constraint for ensuring that an acceptable level of the system performance is achieved. This optimization problem involves choosing the values of time-delays to minimize the biological robustness. Combined constraint transcription with local smoothing approximation techniques, this optimization problem is approximated as a sequence of nonlinear mathematical programming sub-problems. We propose a parallel algorithm, based on the filled function method, for solving these nonlinear programming sub-problems. Numerical results show that the obtained optimal time-delays are highly satisfactory.

The remainder of this paper is organized as follows. In Section 2, a nonlinear time-delay dynamical system is formulated as well as some important properties and strong positive invariance are discussed.. In Section 3, an optimization problem is proposed via biological robustness. In Section 4, computational approaches are presented to solve the optimization problem. In Section 5, numerical results are presented. In Section 6, we draw the conclusions and trace the direction for future works.

\section{Nonlinear time-delay dynamical system}

Glycerol may be converted to 1,3-PD by K. pneumoniae under anaerobic conditions and glycerol dismutation involves two parallel pathways controlled by the dha regulon. The dha regulon is induced by DHA in the absence of an exogenous acceptor. On this basis, a fourteen-dimensional nonlinear dynamic system was presented to describe the batch culture, in which two regulated negative-feedback mechanisms of repression and enzyme inhibition were investigated [25]. In batch culture, a quantity of biomass and glycerol are added to the reactor only once and stirred uniformly under given conditions. During the process of the culture, the concentration of the glycerol decreases gradually and tends to zero finally [26].

In the light of the actual experiment, the following assumptions will be in force throughout the rest of this paper.

H 1. No medium is pumped inside and outside the bioreactor in the process of batch fermentation.

H 2. The concentrations of reactants are uniform in reactor, while nonuniform space distribution is ignored. Nomenclature

- $u_{1}\left(\mu_{m}\right)$ : the maximum specific growth rate in $h^{-1}$.

- $u_{2}\left(K_{s}\right)$ : the Monod saturation constant for substrate in $m m o l L^{-1}$.

- $u_{3}\left(m_{s}\right)$ and $u_{5}\left(m_{1,3-P D}\right), u_{7}\left(m_{H A c}\right), u_{9}\left(m_{E t O H}\right):$ maintenance term of substrate consumption and product (extracellular 1,3-PD, acetate, ethanol) formation under substrate-limited conditions in $\mathrm{mmolg}^{-1} \mathrm{~h}^{-1}$.

- $u_{4}\left(Y_{s}^{m}\right)$ and $u_{6}\left(Y_{1,3-P D}^{m}\right), u_{8}\left(Y_{H A c}^{m}\right), u_{10}\left(Y_{E t O H}^{m}\right):$ the maximum biomass growth yield in gmmol $^{-1}$ and product (extracellular 1,3-PD, acetate, ethanol) yield in $\mathrm{mmolg}^{-1}$.

- $u_{11}\left(U_{G D H t}\right), u_{15}\left(U_{P D O R}\right):$ maintenance term of the specific activity under substrate-limited conditions in $U m g^{-1}$.

- $u_{12}\left(\alpha_{G D H t}\right), u_{16}\left(\alpha_{P D O R}\right):$ constants in enzyme-catalytic kinetics of GDHt and PDOR. 
- $u_{13}\left(\Delta U_{G D H t}\right), u_{17}\left(\Delta U_{P D O R}\right):$ maximum increment of the specific activity under substrate-sufficient conditions in $U m g^{-1}$.

- $u_{14}\left(K_{G D H t}^{s}\right), u_{18}\left(K_{P D O R}^{s}\right):$ saturation constants for the specific activity in kinetic equations with excess terms in $m m o l L^{-1}$.

- $u_{19}\left(V_{s}\right)$ : the specific intracellular volume of cell per biomass in $\mathrm{Lg}^{-1}$.

- $u_{20}\left(\mathcal{J}_{\text {max }}\right):$ a maximum specific transport rate of glycerol in mmol $g^{-1} h^{-1}$.

- $u_{21}\left(K_{m}\right)$ : Michaelis-Menten constant of permease in mmol $L^{-1}$.

- $u_{22}\left(A_{s}=\frac{\delta}{B \times D_{f}}\right)$ : where $B$ denotes the surface area per biomass in $\mathrm{mm}^{2} \mathrm{~g}^{-1} ; D_{f}$ denotes the diffusion coefficient of glycerol in $\mathrm{Lmm}^{-1} h^{-1} ; \delta$ denotes the thickness of cell membrane in $\mathrm{mm}$.

- $u_{23}\left(k_{1}\right), u_{26}\left(k_{2}\right)$ : catalyze coefficient of GDHt for glycerol and PDOR for 3-HPA in $h^{-1}$.

- $u_{24}\left(k_{m G D H t}\right), u_{27}\left(k_{m P D O R}\right):$ Michealis-Menten constant of enzymes of GDHt and PDOR in $m m o l \cdot L^{-1}$.

- $u_{25}\left(k_{i}^{G D H t}\right), u_{28}\left(k_{i}^{P D O R}\right)$ : inhibitor constant for 3-HPA to enzyme of GDHt and PDOR in $\mathrm{mmol} \cdot L^{-1}$.

- $u_{33}\left(K_{P D}\right)$ : a characteristic coefficient of $K$. pneumonia for 1,3-PD diffusion in $h^{-1}$.

- $u_{29}, u_{30}, u_{31}, u_{32}, u_{34}(r), u_{35}\left(K_{M_{R}}\right), u_{36}\left(K_{\theta}\right), u_{37}, u_{38}, u_{39}\left(K_{R}\right), u_{40}\left(K_{d}\right), u_{41}\left(K_{G D H t}^{m}\right), u_{42}\left(K_{d}^{G D H t}\right), u_{43}\left(K_{d}^{m P D O R}\right)$, $u_{44}\left(K_{P D O R}^{m}\right), u_{45}\left(K_{d}^{P D O R}\right):$ non-kinetic parameters.

\subsection{System formulation}

For convenience, let $I_{n}:=\{1,2, \ldots, n\} .[0, T] \subset \mathbb{R}_{+}$(where $\mathbb{R}_{+}$denotes the set of nonnegative real number) be the interval of reaction time. $x_{0}:=\left[x_{01}, x_{02}, \cdots, x_{014}\right] \in \mathbb{R}_{+}^{14}$ is the initial state. Let $x(t)=\left[x_{1}(t), \ldots, x_{14}(t)\right]^{\mathrm{T}} \in \mathbb{R}_{+}^{14}$ (where $A^{\mathrm{T}}$ denotes the transposition of matrix or vector $A$ ) be the continuous state vector, where $x_{1}(t), \ldots, x_{14}(t)$ denote the concentrations of extracellular substances (biomass, extracellular glycerol, extracellular 1,3-PD, acetate, ethanol) and intracellular substances (intracellular glycerol, 3-HPA, intracellular 1,3-PD, $m_{R}$ (mRNA coding repressor), R(free repressor), mGDHt, GDHt, mPDOR, PDOR) at time $t \in[0, T]$, respectively. To simplify notation, we denote $x_{i}(t)$ as $x_{i}, i \in I_{14}$.

Generally, time-delays are deliberately introduced to help stabilize the system or force the system to track real data. In other words, the popularity of the time-delay system is due to the process of substrate absorption together with the inhibitions of substrate and multi-products across the cell membrane. Another advantage of the time-delay system is that such a system can characterize the process by which cells have to undergo growth process before they produce products [59]. Therefore, it is necessary to include the time-delay vector $\tau:=\left[\tau_{3}, \tau_{6}, \tau_{7}, \tau_{8}\right]^{\mathrm{T}} \in \mathbb{R}_{+}^{4}, 0<\tau_{i}<$ $\hat{\tau}_{i}, i=3,6,7,8$, which need to be determined, for extracellular 1,3-PD, intracellular glycerol, 3-HPA and intracellular 1,3-PD in modeling the fermentation process. From [25], under the assumptions $\mathbf{H 1}$ and $\mathbf{H 2}$, mass balance of biomass, substrate and products in batch culture can be characterized as the following nonlinear state-delay dynamical system,

$$
\left\{\begin{array}{l}
\dot{x}(t)=f\left(x(t), x_{\tau}(t), u\right), t \in[0, T], \\
x(t)=\phi(t), \quad t=[-\bar{\tau}, 0), \\
x(0)=x_{0},
\end{array}\right.
$$

where $\bar{\tau}=\max _{i=3,6,7,8} \hat{\tau}_{i} ; \phi(t) \in C^{1}\left([-\bar{\tau}, 0), \mathbb{R}_{+}^{14}\right)$ is a given initial function, in which $C^{1}\left([-\bar{\tau}, 0), \mathbb{R}_{+}^{14}\right)$ is the Banach space of continuously differentiable functions mapping the interval $[-\bar{\tau}, 0)$ into $\mathbb{R}_{+}^{14} ; x_{\tau}(t)=\left[x\left(t-\tau_{3}\right), x\left(t-\tau_{6}\right), x\left(t-\tau_{7}\right), x(t-\right.$ $\left.\left.\tau_{8}\right)\right] \in \mathbb{R}_{+}^{14 \times 4}$, is of the form

$$
\left\{\begin{aligned}
x\left(t-\tau_{3}\right) & =\left[x_{1}(t), x_{2}(t), x_{3}\left(t-\tau_{3}\right), x_{4}(t), \ldots, x_{14}(t)\right]^{\mathrm{T}}, \\
x\left(t-\tau_{6}\right) & =\left[x_{1}(t), \ldots, x_{5}(t), x_{6}\left(t-\tau_{6}\right), x_{7}(t), \ldots, x_{14}(t)\right]^{\mathrm{T}}, \\
x\left(t-\tau_{7}\right) & =\left[x_{1}(t), \ldots, x_{6}(t), x_{7}\left(t-\tau_{7}\right), x_{8}(t), \ldots, x_{14}(t)\right]^{\mathrm{T}}, \\
x\left(t-\tau_{8}\right) & =\left[x_{1}(t), \ldots, x_{7}(t), x_{8}\left(t-\tau_{8}\right), x_{9}(t), \ldots, x_{14}(t)\right]^{\mathrm{T}} .
\end{aligned}\right.
$$


The values of the system parameter vector $u \in \mathbb{R}^{45}$ are uncertain and difficult to determine exactly. Methods for estimating the values of these uncertain parameters using experimental data are given in [10], [25] and [26]. We will use these estimates listed in Table 1 as nominal parameter values in this paper.

Table 1. The nominal values $u^{0}$ of system parameter vector $u$ for system (1) listed in [10], [25] and [26].

\begin{tabular}{cccccccccc}
\multicolumn{8}{c}{ Table 1. The nominal values $u^{0}$ of system parameter vector $u$ for system (1) listed in [10], [25] and [26]. } \\
\hline$u_{1}^{0}$ & $u_{2}^{0}$ & $u_{3}^{0}$ & $u_{4}^{0}$ & $u_{5}^{0}$ & $u_{6}^{0}$ & $u_{7}^{0}$ & $u_{8}^{0}$ & $u_{9}^{0}$ & $u_{10}^{0}$ \\
\hline 0.67 & 0.28 & 2.2 & 0.82 & -2.69 & 67.67 & -0.97 & 33.01 & 5.26 & 11.66 \\
$u_{11}^{0}$ & $u_{12}^{0}$ & $u_{13}^{0}$ & $u_{14}^{0}$ & $u_{15}^{0}$ & $u_{16}^{0}$ & $u_{17}^{0}$ & $u_{18}^{0}$ & $u_{19}^{0}$ & $u_{20}^{0}$ \\
2.8 & 3.53 & 0.87 & 2.41 & 2.32 & 1.87 & 1.38 & 1.35 & 0.151 & 54.664 \\
$u_{21}^{0}$ & $u_{22}^{0}$ & $u_{23}^{0}$ & $u_{24}^{0}$ & $u_{25}^{0}$ & $u_{26}^{0}$ & $u_{27}^{0}$ & $u_{28}^{0}$ & $u_{29}^{0}$ & $u_{30}^{0}$ \\
1.34 & $1.896 \times 10^{-4}$ & 36.3625 & 0.53 & 220.319 & 42.5261 & 0.14 & 0.418 & 1.76 & 880 \\
$u_{31}^{0}$ & $u_{32}^{0}$ & $u_{33}^{0}$ & $u_{34}^{0}$ & $u_{35}^{0}$ & $u_{36}^{0}$ & $u_{37}^{0}$ & $u_{38}^{0}$ & $u_{39}^{0}$ & $u_{40}^{0}$ \\
14.4184 & 20 & 25.177 & 13.1567 & 0.1096 & 0.2004 & 0.0055 & 0.011 & 113050 & 917.9722 \\
$u_{41}^{0}$ & $u_{42}^{0}$ & $u_{43}^{0}$ & $u_{44}^{0}$ & $u_{45}^{0}$ & & & & & \\
10.8083 & 12.7873 & 11.4334 & 24.103 & 20.231 & & & & & \\
\hline
\end{tabular}

Time delay $\tau \in \mathbb{R}_{+}^{4}$ is assumed to be non-negative and bounded, that is,

$$
\tau \in \mathcal{T}:=\left[0, \hat{\tau}_{3}\right] \times\left[0, \hat{\tau}_{6}\right] \times\left[0, \hat{\tau}_{7}\right] \times\left[0, \hat{\tau}_{8}\right] \subseteq \mathbb{R}_{+}^{4} .
$$

The right hand side of the system (1) is of the form $f\left(x(t), x_{\tau}(t), u\right):=\left[f_{1}\left(x(t), x_{\tau}(t), u\right), \ldots, f_{14}\left(x(t), x_{\tau}(t), u\right)\right]^{\mathrm{T}}$ with the components defined by

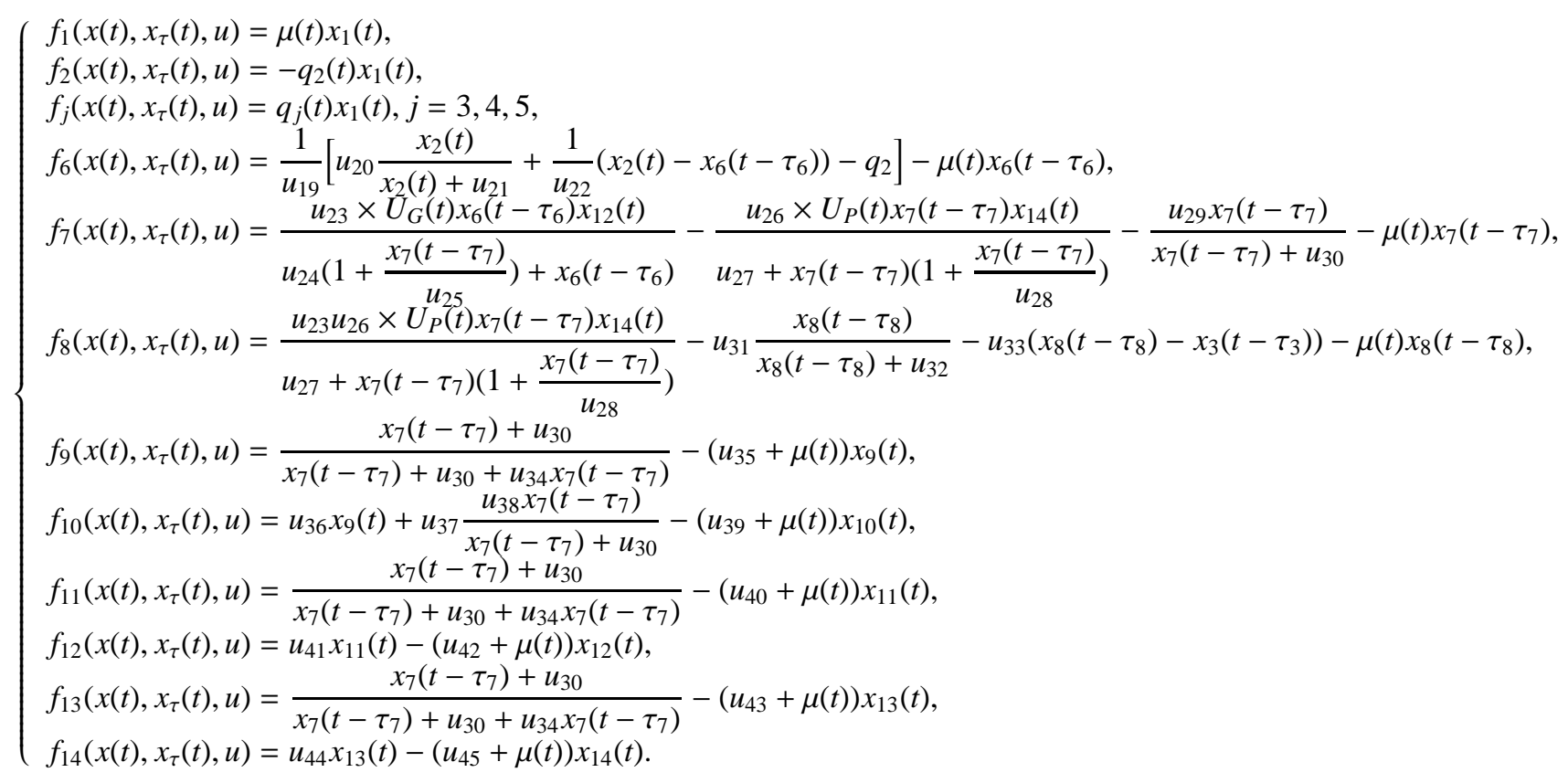


The specific substrate consumption rate and the specific product formation rate can be expressed as follows:

$$
\left\{\begin{array}{l}
\mu(t)=\frac{u_{1} x_{2}(t)}{x_{2}(t)+u_{2}}\left(1-\frac{x_{3}\left(t-\tau_{3}\right)}{x_{3}^{*}}\right)\left(1-\frac{x_{7}\left(t-\tau_{7}\right)}{x_{7}^{*}}\right) \prod_{j=2, j \neq 3}^{5}\left(1-\frac{x_{j}(t)}{x_{j}^{*}}\right) \\
q_{2}(t)=u_{3}+\frac{\mu(t)}{u_{4}} \\
q_{3}(t)=u_{5}+\mu(t) u_{6} \\
q_{4}(t)=u_{7}+\mu(t) u_{8} \\
q_{5}(t)=u_{9}+\mu(t) u_{10} \\
U_{G}(t)=u_{11}-u_{12} \mu(t)-u_{13} \frac{x_{2}(t)}{x_{2}(t)+u_{14}} \\
U_{P}(t)=u_{15}-u_{16} \mu(t)-u_{17} \frac{x_{2}(t)}{x_{2}(t)+u_{18}}
\end{array}\right.
$$

Since each component of the state vector $x$ represents a certain substance concentration, the concentrations of biomass, glycerol and products should be restricted in a certain range according to the practical fermentation process. On the basis of the results in [25], the admissible set $W$ of $x(t)$ is defined by

$$
x(t) \in W:=\prod_{i=1}^{14}\left[x_{i *}, x_{i}^{*}\right] \subset \mathbb{R}_{+}^{14},
$$

with

$x_{*}=[0.0001,0.01,0,0,0,0,0,0,0,0,0,0,0,0]^{\mathrm{T}}, x^{*}=[5,2039,939.5,1026,360.9,2039,300,939.5, M, M, M, M, M, M]^{\mathrm{T}}$, where $M$ is a large enough positive number.

\subsection{Fundamental property of system}

Similarly to [51], we have the following properties.

Property 1. The function $f: \mathbb{R}_{+}^{14} \times \mathbb{R}_{+}^{14 \times 4} \times \mathbb{R}^{45} \rightarrow \mathbb{R}^{14}$, in the system modeled by (1) satisfies the following conditions:

(I) $f\left(x(t), x_{\tau}(t), u\right)$ is Lipschitz continuous in $x, x_{\tau}$;

(II) There exist $\bar{\zeta}>0$, such that

$$
\left\|f\left(x(t), x_{\tau}(t), u\right)\right\| \leq \bar{\zeta}\left(\|x(t)\|+\left\|x_{\tau}(t)\right\|+1\right),
$$

where $\|\cdot\|$ is a Euclidean norm.

Property 2. Given $u \in \mathbb{R}^{45}$, for each $\tau \in \mathcal{T}$, the system $(1)$ with initial condition $x(0) \in W$ and $\phi(t) \in C^{1}\left([-\bar{\tau}, 0), \mathbb{R}_{+}^{14}\right)$ has a unique continuous solution, denoted by $x(\cdot \mid \tau, u)$. Furthermore, $x(\cdot \mid \tau, u)$ satisfies that

$$
x(t \mid \tau, u):=x(0)+\int_{0}^{t} f\left(x(s \mid \tau, u), x\left(s-\tau_{3} \mid \tau, u\right), x\left(s-\tau_{6} \mid \tau, u\right), x\left(s-\tau_{7} \mid \tau, u\right), x\left(s-\tau_{8} \mid \tau, u\right), u\right) d s, \forall t \in[0, T]
$$

and $x(t \mid \tau, u)=\phi(t), \forall t \in[-\bar{\tau}, 0)$.

Property 3. Given $u \in \mathbb{R}^{45}, \phi(t) \in C^{1}\left([-\bar{\tau}, 0), \mathbb{R}_{+}^{14}\right)$ and $x(0) \in W$, the solution $x(t \mid \tau$, $u)$ of the system (1) is uniformly bounded and Lipschitzian. 


\subsection{Strong positive invariance}

For the given system parameter vector $u \in \mathbb{R}^{45}$, we define the set-valued mapping $F: \mathbb{R}^{14} \rightrightarrows \mathbb{R}^{14}$

$$
F(x):=\left\{f\left(x(t), x_{\tau}(t), u\right), t \in[0, T] \mid \tau \in \mathcal{T}\right\} .
$$

It follows from Properties 1-3 that it is tedious, but not difficult, to verify the following properties for $F(\cdot)$ :

1. For each $x \in \mathbb{R}_{+}^{14}, F(x)$ is a compact set.

2. $F(x)$ is upper semi-continuous and locally Lipschitz on $\mathbb{R}_{+}^{14}$, respectively.

3. For $\forall x \in \mathbb{R}_{+}^{14}$,

$$
\bar{v} \in F(x) \Rightarrow\|\bar{v}\| \leq \bar{\gamma}\|x\|+\bar{c},
$$

where $\bar{\gamma}>0$ and $\bar{c}>0$ are constants.

Definition 1. [62] The system $\left(\mathbb{R}_{+}^{14}, F(x)\right)$ is said to be strongly positive invariant if every trajectory $x$ on $[0, \infty)$ for which $x(0) \in W$ and $\phi(t) \in C^{1}\left([-\bar{\tau}, 0), \mathbb{R}_{+}^{14}\right)$ are such that $x(t \mid \tau, u) \in W$ for all $t>0$.

The upper Hamiltonian [62] $H$ corresponding to $F$ from $\mathbb{R}^{14} \times \mathbb{R}^{14}$ to $\mathbb{R}$ is defined as follows:

$$
H(x, p):=\max _{v \in F(x)}\langle p, v\rangle,
$$

where $p \in \mathbb{R}^{14}$ and $\left.\langle\cdot, \cdot\rangle\right\rangle$ is the Euler inner product.

The proximal normal cone [62] at $x \in \mathbb{R}_{+}^{14}$ is defined as follows:

$$
N_{\mathbb{R}_{+}^{14}}^{P}(x):=\left\{\zeta: \exists t>0 \text { so that } d_{\mathbb{R}_{+}^{14}}(x+t \zeta)=t\|\zeta\|\right\},
$$

where $d_{\mathbb{R}_{+}^{14}}(x):=\inf \left\{\|x-s\|: s \in \mathbb{R}_{+}^{14}\right\}$.

The normal cone [62] at $x \in \mathbb{R}_{+}^{14}$ is defined as follows:

$$
N_{\mathbb{R}_{+}^{14}}(x):=\left\{\zeta \in\left(\mathbb{R}_{+}^{14}\right)^{\bar{*}}:\langle\zeta, \bar{x}-x\rangle \leq 0, \forall \bar{x} \in \mathbb{R}_{+}^{14}\right\},
$$

where $\left(\mathbb{R}_{+}^{14}\right)^{*}$ is the conjugate space of $\mathbb{R}_{+}^{14}$.

Let int $\mathbb{R}_{+}^{14}$ and $\partial \mathbb{R}_{+}^{14}$ denote the interior and boundary of $\mathbb{R}_{+}^{14}$, respectively.

Define

$$
\begin{aligned}
I_{14}(x) & :=\{1,2,1,1,14\}, \\
\mathcal{F}(x) & :=\left\{j \in I_{14}(x) \mid x_{3}\left(t-\tau_{3}\right)=0, x_{6}\left(t-\tau_{6}\right)=0, x_{7}\left(t-\tau_{7}\right)=0, x_{8}\left(t-\tau_{8}\right)=0, x_{j}=0, j \neq 3,6,7,8\right\}, \\
\mathcal{P}(x) & :=\left\{j \in I_{14}(x) \mid x_{3}\left(t-\tau_{3}\right)>0, x_{6}\left(t-\tau_{6}\right)>0, x_{7}\left(t-\tau_{7}\right)>0, x_{8}\left(t-\tau_{8}\right)>0, x_{j}>0, j \neq 3,6,7,8\right\} .
\end{aligned}
$$

Theorem 1. The system $\left(\mathbb{R}_{+}^{14}, F\right)$ is strongly positive invariant, that is, the system (1) has a unique continuous positive solution (i.e., $x(t \mid \tau, u) \geq 0, \forall t \in[0, T])$, if $x_{02} \geq T\left(u_{3}+u_{1} / u_{4}\right) x_{1}^{*}, u_{5}+u_{1} u_{6} \geq 0, u_{7}+u_{1} u_{8} \geq 0, u_{9}+u_{1} u_{10} \geq$ $0, u_{20} x_{* 2} /\left(x_{2}^{*}+u_{21}\right)+x_{* 2} / u_{22} \geq u_{3}+u_{1} / u_{4}, u_{11} \geq u_{1} u_{12}+u_{13}, u_{14} \geq u_{1} u_{15}+u_{16}, u_{j}>0, j \in I_{45} \backslash\{1,3, \ldots, \ldots, 16,20, \ldots, \ldots, 23\}$. Proof. From Theorem 3.8 of Chapter 4 in [62] and property for $F(\cdot)$, to complete the proof of the theorem, it suffices now to prove that

Case 1: It follows that

$$
H\left(x, N_{\mathbb{R}_{+}^{14}}^{P}(x)\right) \leq 0, \forall x \in \mathbb{R}_{+}^{14},
$$

$$
H\left(x, N_{\mathbb{R}_{+}^{14}}^{P}(x)\right)=0, \forall x \in \operatorname{int} \mathbb{R}_{+}^{14},
$$

since for $x \in \operatorname{int} \mathbb{R}_{+}^{14}, N_{\mathbb{R}_{+}^{14}}^{P}(x)=N_{\mathbb{R}_{+}^{14}}(x)$ and $N_{\mathbb{R}_{+}^{14}}(x)=\{\mathbf{0}\}$. 
Case 2: Clearly, $\mathcal{F}(x) \neq \emptyset$ and

$$
N_{\mathbb{R}_{+}^{14}}^{P}(x)=N_{\mathbb{R}_{+}^{14}}(x)=\left\{\begin{array}{l}
\left\{p \in \mathbb{R}^{14} \mid p_{j} \leq 0, j \in \mathcal{F}(x), p_{k}=0, k \in \mathcal{P}(x)\right\}, x \in \partial \mathbb{R}_{+}^{14} \backslash\{\mathbf{0}\}, \\
\left\{p \in \mathbb{R}^{14} \mid p_{j} \leq 0, j \in I_{14}(x)\right\}, x=\mathbf{0} .
\end{array}\right.
$$

When $x_{02} \geq T\left(u_{3}+u_{1} / u_{4}\right) x_{1}^{*}, u_{5}+u_{1} u_{6} \geq 0, u_{7}+u_{1} u_{8} \geq 0, u_{9}+u_{1} u_{10} \geq 0$, some components of solution to the system (4) satisfies

$$
\left\{\begin{array}{l}
x_{1}(t)=x_{01} \exp \left(\int_{0}^{t} \mu(s) \mathrm{d} s\right) \geq 0, \\
x_{2}(t)=x_{02}-\int_{0}^{t} q_{2}(s) x_{1}(s) \mathrm{d} s \geq 0, \\
x_{j}(t)=x_{0 j}+\int_{0}^{t} q_{j}(s) x_{1}(s) \mathrm{d} s \geq 0, j=3,4,5 .
\end{array}\right.
$$

When $u_{20} x_{* 2} /\left(x_{2}^{*}+u_{21}\right)+x_{* 2} / u_{22} \geq u_{3}+u_{1} / u_{4}$, it follows from (4) that $f_{6}\left(x(t), x_{\tau}(t), u\right) \geq 0, \forall \tau \in \mathcal{T}$, if $x_{6}\left(t-\tau_{6}\right)=0$. Similarly, it follows that $f_{j}\left(x(t), x_{\tau}(t), u\right) \geq 0, \forall \tau \in \mathcal{T}, j \in \mathcal{F}(x) \cap I_{14}(x)$, when $u_{11} \geq u_{1} u_{12}+u_{13}, u_{14} \geq u_{1} u_{15}+u_{16}, u_{j}>$ $0, j \in I_{45} \backslash\{1,3, \ldots, \ldots, 16,20, \ldots, \ldots, 23\}$. That is,

$$
f_{j}\left(x(t), x_{\tau}(t), u\right) \geq 0, \forall \tau \in \mathcal{T}, j \in \mathcal{F}(x)
$$

It follows from (10)-(11) and the definition of $H\left(x, N_{\mathbb{R}_{+}^{14}}^{P}(x)\right)$ that we have

$$
H\left(x, N_{\mathbb{R}_{+}^{11}}^{P}(x)\right) \leq 0, x \in \partial \mathbb{R}_{+}^{14} \backslash\{\mathbf{0}\} .
$$

It follows from (11) that

$$
f_{j}\left(x(t), x_{\tau}(t), u\right) \geq 0, \forall \tau \in \mathcal{T}, j \in I_{14}(x),
$$

if $x_{3}\left(t-\tau_{3}\right)=0, x_{6}\left(t-\tau_{6}\right)=0, x_{7}\left(t-\tau_{7}\right)=0, x_{8}\left(t-\tau_{8}\right)=0, x_{j}=0, j \in I_{14} \backslash\{3,6,7,8\}$. Thus, if follows from (10) that

$$
H\left(x, N_{\mathbb{R}_{+}^{14}}^{P}(x)\right) \leq 0, x_{3}\left(t-\tau_{3}\right)=0, x_{6}\left(t-\tau_{6}\right)=0, x_{7}\left(t-\tau_{7}\right)=0, x_{8}\left(t-\tau_{8}\right)=0, x_{j}=0, j \in I_{14} \backslash\{3,6,7,8\} .
$$

It follows from (9), (12), (14), Theorem 3.8 of Chapter 4 in [62] and property for $F(\cdot)$ that this completes the proof.

\section{Biological robustness and optimization problem}

The concentrations of extracellular substances can be measured in experiments, but note that we can only obtain experimental data for these substances. Since it is difficult to measure the concentrations of intracellular substances, we quantitatively define biological robustness based on numerical characteristics (expectation and variance). The purpose of this section is to consider an optimization problem to determine the optimal values for time-delay vector such that some states of the system (1) better fit given experimental data and the quantitative biological robustness is minimized.

\subsection{Relative error}

In the fermentation process, alkali, which is intermittently fed into the reactor to maintain its $\mathrm{pH}$ value at 7.0 or so, can chemically react with acetic acid and ethanol. This operation has great additional effect on the concentrations of acetic acid and ethanol from the system (1). This leads to the inaccuracy of the concentrations of acetic acid and ethanol. For this, this paper is concerned with the relative error between experimental data and computational values of the first three substances (i.e. biomass, extracellular glycerol, extracellular 1,3-PD) [17].

Let $y(t)=\left[y_{1}(t), y_{2}(t), y_{3}(t)\right] \in C^{1}\left([0, T] ; \mathbb{R}^{3}\right)$ denote the vector function fitted by experimental data with initial state $x_{0} \in W$, namely, its components denote the concentration of extracellular substances at time point $t \in[0, T]$. 
Definition 2. For the given system parameter vector $u \in \mathbb{R}^{45}$, the relative error $\operatorname{SRE}(\tau \mid u)$ between the solution $x_{j}(t \mid \tau, u), j \in I_{3}$ and experimental concentrations $y_{j}(t), j \in I_{3}$ can be defined by

$$
\operatorname{SRE}(\tau \mid u):=\frac{1}{3 \cdot T} \sum_{j=1}^{3} \int_{0}^{T} \frac{\left[x_{j}(t \mid \tau, u)-y_{j}(t)\right]^{2}}{\left[y_{j}(t)\right]^{2}} \mathrm{~d} t
$$

For the given system parameter vector $u \in \mathbb{R}^{45}$, the feasible set of time-delay vector $\tau$ is defined as

$$
\mathcal{T}_{W}:=\left\{\tau \in \mathcal{T} \mid \operatorname{SRE}(\tau \mid u) \leq \sigma_{1}, x(t \mid \tau, u) \in W, \forall t \in[0, T]\right\} .
$$

where $\sigma_{1}>0$ is a given small constant.

According to the actual fermentation process, $\mathcal{T}_{W}$ is not a empty set. Therefore, we assume that

H 3. $\mathcal{T}_{W}$ is a nonempty set.

It follows from the compactness of $\mathcal{T}$ and $W$ together with the boundedness of the solution $x(\cdot \mid \tau, u)$ that we have the following property:

Property 4. Under assumption $\mathbf{H 3}, \mathcal{T}_{W}$ is a compact set.

\subsection{Biological robustness}

Due to lack of precise experimental data for intracellular substances (i.e. $\left.x_{j}(\cdot \mid \tau, u), j=6, \ldots, 14\right)$, the task of this section is how to judge the reliability of numerical solution to the system (1) for intracellular substances. To this end, the quantified biological robustness is a popular method to address this task. The definition of biological robustness for batch culture should meet the case that the smaller the changes in state variables provoked by small shift in unknown parameters for the overall process of batch culture, the more the system's performance is robust [46]. We have quantitatively defined the biological robustness of batch culture in our previous work [26], which has one limitation: this definition only considers the expectation of the relative deviation between system outputs before and after system parameters are perturbed.

Variance is a concept that describes, in part, either the actual probability distribution of an observed population of numbers, or the theoretical probability distribution of a not-fully-observed population from which a sample of numbers has been drawn [60]. Variance is always non-negative: a small variance indicates that the data points tend to be very close to the mean (expected value) and hence to each other, while a high variance indicates that the data points are very spread out around the mean and from each other. With this motivation, inspired by the qualitative description of the biological robustness [41], the quantitative biological robustness of the intracellular substances concentrations, which is different from biological robustness in [52], is defined by penalizing a weighted sum of the expectation and variance of the relative deviation between system outputs before and after time-delays are perturbed.

For given $\bar{\delta}>0\left(\bar{\delta}\right.$ is the convergence tolerance for the relative deviation of $\left.\tau_{j}, j=3,6,7,8\right)$ and $\tau \in \mathcal{T}_{W}$, we define the rectangular field of dimensional normalization, denoted by $B(\tau, \bar{\delta})$, as follows:

$$
B(\tau, \bar{\delta}):=\left\{\check{\tau} \in \mathcal{T}_{W} \mid \frac{\left|\check{\tau}_{j}-\tau_{j}\right|}{\left|\tau_{j}\right|} \leq \bar{\delta}, j=3,6,7,8\right\} .
$$

For the given system parameter vector $u \in \mathbb{R}^{45}, V S(\tau), \tau \in \mathcal{T}_{W}$ (the feasible set of time-delay vector), is defined by

$$
V S(\tau):=\{x(\cdot \mid \tilde{\tau}, u) \mid x(\cdot \mid \tilde{\tau}, u) \text { is the solution of system (1) corresponding to } \tilde{\tau} \in B(\tau, \bar{\delta})\}, \tau \in \mathcal{T}_{W} .
$$

For the given parameter vector $u \in \mathbb{R}^{45}$ and $t \in[0, T]$, the relative deviation between the solution $x_{j}(t \mid \tilde{\tau}, u) \in$ $V S(\tau), \tau \in \mathcal{T}_{W}$ with respect to $\tilde{\tau} \in B(\tau, \bar{\delta}) \subseteq \mathcal{T}_{W}$, and the solution $x_{j}(t \mid \tau, u), j=6, \ldots, 14, \tau \in \mathcal{T}_{W}$, is defined by

$$
\operatorname{SRD}_{j}(x(t \mid \tilde{\tau}, u), x(t \mid \tau, u)):=\frac{\left[x_{j}(t \mid \tilde{\tau}, u)-x_{j}(t \mid \tau, u)\right]^{2}}{\left[x_{j}(t \mid \tau, u)\right]^{2}} \frac{1}{\frac{\|\tilde{\tau}-\tau\|}{\|\tau\|}} .
$$


For the given system parameter vector $u \in \mathbb{R}^{45}$, the expectation and variance of the relative deviation $S R D_{j}(x(t \mid \tilde{\tau}, u), x(t \mid \tau, u)), j=$ $6, \ldots, 14$, caused by $\tilde{\tau}$ uniformly distributed in field of $B(\tau, \bar{\delta})$ are respectively defined by

$$
\begin{aligned}
& E_{j}\left(t \mid S R D_{j}, \tau, u\right):=\int_{\Omega} \Psi(\tilde{\tau}-\tau) S R D_{j}(x(t \mid \tilde{\tau}, u), x(t \mid \tau, u)) \mathrm{d}(\tilde{\tau}-\tau), j=6, \ldots, 14, \\
& D_{j}\left(t \mid S R D_{j}, \tau, u\right):=E_{j}\left(t \mid S R D_{j} \times S R D_{j}, \tau, u\right)-\left[E_{j}\left(t \mid S R D_{j}, \tau, u\right)\right]^{2}, j=6, \ldots, 14,
\end{aligned}
$$

where $\Omega \subset \mathcal{T}_{W}$ is a perturbation space with respect to $\tau \in \mathcal{T}_{W}$ and $\Psi(\tilde{\tau}-\tau)$ is taken as an equal probability in $\tilde{\tau}-\tau$ on $\Omega$.

Definition 3. For the given system parameter vector $u \in \mathbb{R}^{45}$, the cost function, i.e., the biological robustness of the system (1) is defined by

$$
J(\tau \mid u):=\sum_{j=6}^{14} \int_{0}^{T}\left[E_{j}\left(t \mid S R D_{j}, \tau, u\right)+w D_{j}\left(t \mid S R D_{j}, \tau, u\right)\right] \mathrm{d} t,
$$

where $w \geq 0$ is a given weight.

Remark 1. The smaller the value of (22) is, the more robust the system (1) is. That is, given $u \in \mathbb{R}^{45}$, the system (1) with respect to its time-delay vector $\tau^{1}$ can be said to be more robust than with respect to its other time-delay vector $\tau^{2} \neq \tau^{1}$, if

$$
J\left(\tau^{1} \mid u\right)<J\left(\tau^{2} \mid u\right)
$$

\subsection{Optimization problem via biological robustness}

Most of the existing control and synchronization methods for nonlinear time-delay systems are only effective when all model parameters are known. However, in practice, it is common for the values of some time-delays to be unknown. Thus, before designing a control scheme to realize synchronization, the unknown time-delays must first be identified.

In the process of fermentation, the system parameter vector $u$ is usually not known exactly and thus needs to be estimated. We suppose that $u$ is a given system parameter vector whose components are nominal estimates of the parameter vector from (4) to (5). That is, the nominal estimates are only approximations of the true parameter vector. After the optimal $\tau^{*}$ is obtained, an important issue that then arises is whether system performance under the optimal $\tau^{*}$ is sensitive to errors in $u$. Thus, inspired by the work in [47, 48, 49], we define the following cost sensitivity corresponding to the vector $u$ as

$$
\left[\frac{\partial J(\tau \mid u)}{\partial u}\right]\left[\frac{\partial J(\tau \mid u)}{\partial u}\right]^{\mathrm{T}} .
$$

Clearly, (23) measures the rate at which the process yield changes in response to small changes in the system parameter vector $u$. Thus, a low value of the cost sensitivity indicates that the optimal time-delays obtained are not sensitive to the variation of the system parameter vector.

In view of this concern, we propose to formulate an optimization problem, where the biological robustness (22) is minimized subject to a quality constraint, a cost sensitivity constraint, continuous state inequality constraints and bound constraints. It is formally stated as follows.

$$
\begin{array}{cll}
\text { Problem (TDOP) : } \min & J(\tau \mid u) \\
\text { s.t. } \quad & S R E(\tau \mid u) \leq \sigma_{1}, \\
& {\left[\frac{\partial J(\tau \mid u)}{\partial u}\right]\left[\frac{\partial J(\tau \mid u)}{\partial u}\right]^{\mathrm{T}} \leq \sigma_{2},} \\
& x(t \mid \tau, u) \in W, \forall t \in[0, T], \\
& \tau \in \mathcal{T}, \\
10 &
\end{array}
$$


where (24) is a quality constraint for ensuring an acceptable level of the time-delay system better fits given experimental data; (25) is a cost sensitivity constraint for ensuring an acceptable level of the system performance is achieved; (26) is termed the continuous state inequality constraint [53], which must be satisfied at every point in the time horizon $[0, T] ; \sigma_{1}>0$ and $\sigma_{2}>0$ are given small constants.

For the given parameter vector $u \in \mathbb{R}^{45}$, a set $\mathcal{T}_{J}$ is defined by

$$
\mathcal{T}_{J}:=\left\{\tau \in \mathcal{T}_{W} \mid\left[\frac{\partial J(\tau \mid u)}{\partial u}\right]\left[\frac{\partial J(\tau \mid u)}{\partial u}\right]^{\mathrm{T}} \leq \sigma_{2}\right\}
$$

In view of the actual fermentation process, $\mathcal{T}_{J}$ is not an empty set. Therefore, we assume that

H 4. $\mathcal{T}_{J}$ is not an empty set.

It follows from Property 4 that we have the following property:

Property 5. Under assumption $\mathbf{H 4}, \mathcal{T}_{J}$ is a compact set.

The existence of the optimal solution to Problem TDOP is stated as follows.

Theorem 2. Given parameter vector $u \in \mathbb{R}^{45}$, Problem TDOP has at least one optimal solution.

Proof. It follows from the continuity of the cost function in (22) and the compactness of the set $\mathcal{T}_{J}$ that this completes the proof.

\section{Computational approaches}

In this section, we shall develop a numerical solution method to solve Problem TDOP.

\subsection{Computing cost sensitivity}

We now derive a formula for the cost sensitivity of Problem TDOP in the following theorem.

Theorem 3. Then, for each $\tau \in \mathcal{T}_{J}, w \geq 0$,

$$
\left[\frac{\partial J(\tau \mid u)}{\partial u}\right]\left[\frac{\partial J(\tau \mid u)}{\partial u}\right]^{\mathrm{T}}=\sum_{k=1}^{45}\left\{\frac{\partial J(\tau \mid u)}{\partial u_{k}}\right\}^{2}=\sum_{k=1}^{45}\left\{\sum_{j=6}^{14} \int_{0}^{T}\left[\frac{\partial E_{j}\left(t \mid S R D_{j}, \tau, u\right)}{\partial u_{k}}+w \frac{\partial D_{j}\left(t \mid S R D_{j}, \tau, u\right)}{\partial u_{k}}\right] \mathrm{d} t\right\}^{2},
$$

with

$$
\begin{gathered}
\frac{\partial E_{j}\left(t \mid S R D_{j}, \tau, u\right)}{\partial u_{k}}=\int_{\Omega} \Psi(\tilde{\tau}-\tau) \frac{\partial S R D_{j}(x(t \mid \tilde{\tau}, u), x(t \mid \tau, u))}{\partial u_{k}} \mathrm{~d}(\tilde{\tau}-\tau), j=6, \ldots, 14, k \in I_{45}, \\
\frac{\partial D_{j}\left(t \mid S R D_{j}, \tau, u\right)}{\partial u_{k}}=2 \int_{\Omega} \Psi(\tilde{\tau}-\tau) S R D_{j}(x(t \mid \tilde{\tau}, u), x(t \mid \tau, u)) \frac{\partial S R D_{j}(x(t \mid \tilde{\tau}, u), x(t \mid \tau, u))}{\partial u_{k}} \mathrm{~d}(\tilde{\tau}-\tau) \\
+2 E_{j}\left(t \mid S R D_{j}, \tau, u\right) \frac{\partial E_{j}\left(t \mid S R D_{j}, \tau, u\right)}{\partial u_{k}}, j=6, \ldots, 14, k \in I_{45},
\end{gathered}
$$

and

$$
\frac{\partial S R D_{j}(x(t \mid \tilde{\tau}, u), x(t \mid \tau, u))}{\partial u_{k}}=\frac{1}{\left\|\frac{\tilde{\tau}-\tau}{\tau}\right\|} \frac{\left(x_{j}(t \mid \tilde{\tau}, u)-x_{j}(t \mid \tau, u)\right)}{\left[x_{j}(t \mid \tau, u)\right]^{2}}\left\{2\left(\lambda^{j, k}(t \mid \tilde{\tau}, u)-\lambda^{j, k}(t \mid \tau, u)\right)-\left(x_{j}(t \mid \tilde{\tau}, u)-x_{j}(t \mid \tau, u)\right) \lambda^{j, k}(t \mid \tau, u)\right\},
$$

where $\lambda(t \mid \tau, u)=\left(\lambda^{j, k}(t \mid \tau, u)\right)_{14 \times 45} \in \mathbb{R}^{14 \times 45}$, is the solution of the following auxiliary delay-differential system:

$$
\begin{aligned}
\dot{\lambda}(t \mid \tau, u)= & \frac{\partial f\left(x(t \mid \tau, u), x_{\tau}, u\right)}{\partial x(t \mid \tau, u)} \lambda(t \mid \tau, u)+\sum_{j=3,6,7,8} \frac{\partial f\left(x(t \mid \tau, u), x_{\tau}, u\right)}{\partial x\left(t-\tau_{j} \mid \tau, u\right)} \lambda\left(t-\tau_{j} \mid \tau, u\right) \\
& +\frac{\partial f\left(x(t \mid \tau, u), x_{\tau}, u\right)}{\partial u}, \forall t \in[0, T],
\end{aligned}
$$


with

$$
\lambda(t \mid \tau, u)=\mathbf{0} \in \mathbb{R}^{14 \times 45}, \quad \forall t \in[-\bar{\tau}, 0) .
$$

Proof. Let $\tau \in \mathcal{T}_{J}$ be an admissible time-delay. By Theorem 3.2 in [32], we have

$$
\frac{\partial x_{j}(t \mid \tau, u)}{\partial u_{k}}=\lambda^{j, k}(t \mid \tau, u), j \in I_{14}, k \in I_{45},
$$

where $\lambda(t \mid \tau, u)=\left(\lambda^{j, k}(t \mid \tau, u)\right)_{14 \times 45} \in \mathbb{R}^{14 \times 45}$, is the solution of systems (30) and (31). Thus, differentiating $J(\tau \mid u)$ with respect to $u_{k}$ yields

$$
\frac{\partial J(\tau \mid u)}{\partial u_{k}}=\sum_{j=6}^{14} \int_{0}^{T}\left[\frac{\partial E_{j}\left(t \mid S R D_{j}, \tau, u\right)}{\partial u_{k}}+w \frac{\partial D_{j}\left(t \mid S R D_{j}, \tau, u\right)}{\partial u_{k}}\right] \mathrm{d} t, k \in I_{45}
$$

Hence,

$$
\left[\frac{\partial J(\tau \mid u)}{\partial u}\right]\left[\frac{\partial J(\tau \mid u)}{\partial u}\right]^{\mathrm{T}}=\sum_{k=1}^{45}\left\{\frac{\partial J(\tau \mid u)}{\partial u_{k}}\right\}^{2}=\sum_{k=1}^{45}\left\{\sum_{j=6}^{14} \int_{0}^{T}\left[\frac{\partial E_{j}\left(t \mid S R D_{j}, \tau, u\right)}{\partial u_{k}}+w \frac{\partial D_{j}\left(t \mid S R D_{j}, \tau, u\right)}{\partial u_{k}}\right] \mathrm{d} t\right\}^{2} \cdot \square
$$

\subsection{Approximate problems}

To handle the continuous state inequality constraint (26), let

$$
g_{i}(x(t \mid \tau, u)):=x_{i}^{*}-x_{i}(t \mid \tau, u), \quad g_{i+14}(x(t \mid \tau, u)):=x_{i}(t \mid \tau, u)-x_{i *}, i \in I_{14} .
$$

The constraint (26) is equivalently transcribed into

$$
G^{i}(\tau \mid u)=0, i \in I_{28}
$$

where $G^{i}(\tau \mid u):=\int_{0}^{T} \min \left\{0, g_{i}(x(t \mid \tau, u))\right\} \mathrm{d} t, i \in I_{28}$. However, $G^{i}(\tau \mid u), i \in I_{28}$, is non-smooth-a non-desirable trait that poses a problem for conventional optimization algorithms, in $\tau \in \mathcal{T}_{J}$. In addition, the equality constraints specified in (33) do not satisfy the constraint qualification. Thus it is not advisable to compute it as such numerically. Hence, we replace (33) with

$$
\tilde{G}_{\varepsilon, \gamma}^{i}(\tau \mid u):=\int_{0}^{T} \varphi_{\varepsilon, i}\left(g_{i}(x(t \mid \tau, u))\right) d t+\gamma \geq 0, i \in I_{28},
$$

where $\varepsilon>0, \gamma>0$ and

$$
\varphi_{\varepsilon, i}\left(g_{i}(x(t \mid \tau, u))\right):= \begin{cases}g_{i}(x(t \mid \tau, u)), & \text { if } g_{i}(x(t \mid \tau, u)) \in(-\infty,-\varepsilon), \\ -\frac{\left[g_{i}(x(t \mid \tau, u))-\varepsilon\right]^{2}}{4 \varepsilon}, & \text { if } g_{i}(x(t \mid \tau, u)) \in[-\varepsilon, \varepsilon] \\ 0, & \text { if } g_{i}(x(t \mid \tau, u)) \in(\varepsilon,+\infty)\end{cases}
$$

Then, replacing (26) with (34) yields dynamic optimization problem with a finite number of canonical constraints. That is, Problem TDOP can be approximated by a sequence of nonlinear programming Problems $\left\{\mathbf{T D O P}_{\varepsilon, \gamma}\right\}$. The relationship between Problems $\left\{\mathbf{T D O P}_{\varepsilon, \gamma}\right\}$ and Problem TDOP is stated in the following theorem, the proof of which is given in Appendix A. 
Theorem 4. There exists a $\gamma(\varepsilon)>0$ such that for all $\gamma, 0<\gamma<\gamma(\varepsilon)$, any feasible time-delay $\tau_{\varepsilon, \gamma}$ to Problem $\mathbf{T D O P}_{\varepsilon, \gamma}$, i.e.,

$$
\begin{aligned}
\operatorname{SRE}\left(\tau_{\varepsilon, \gamma} \mid u\right) & \leq \sigma_{1}, \\
\left|\left[\frac{\partial J\left(\tau_{\varepsilon, \gamma} \mid u\right)}{\partial u}\right]\left[\frac{\partial J\left(\tau_{\varepsilon, \gamma} \mid u\right)}{\partial u}\right]^{\mathrm{T}}\right| & \leq \sigma_{2}, \\
\tilde{G}_{\varepsilon, \gamma}^{i}\left(\tau_{\varepsilon, \gamma} \mid u\right)=\int_{0}^{T} \varphi_{\varepsilon, i}\left(g_{i}\left(x\left(t \mid \tau_{\varepsilon, \gamma}, u\right)\right)\right) d t+\gamma & \geq 0, i \in I_{28} .
\end{aligned}
$$

is also a feasible point of Problem TDOP.

Remark 2. This theorem ensures that the corresponding $\gamma(\varepsilon)$ for each $\varepsilon$ in this sequence is finite.

For constructing an optimization algorithm, when the time-delay vector $\tau \in \mathcal{T}_{J}$ is not feasible, we can move $\tau$ towards the feasible region in the positive direction of the gradients of constraint $\tilde{G}_{\varepsilon, \gamma}^{i}(\tau \mid u), i \in I_{28}$ with respect to time-delay vector $\tau \in \mathcal{T}_{J}$. The main challenge of calculating such gradients is that the auxiliary time-delay systems can be solved simultaneously with the system (1). Define

$$
\chi(t):= \begin{cases}\dot{\phi}(t), & \text { if } t \leq 0, \\ f\left(x, x_{\tau}, u\right), & \text { if } t \in(0, T] .\end{cases}
$$

In the process of constructing the optimization algorithm, the gradients of constraint $\tilde{G}_{\varepsilon, \gamma}^{i}(\tau \mid u), i \in I_{28}$ with respect to $\tau \in \mathcal{T}_{J}$ are needed. However, the traditional approaches for computing the gradient of the constraint $\tilde{G}_{\varepsilon, \gamma}^{i}(\tau \mid u), i \in I_{28}$ involves integrating two systems (the state system and the costate system) of differential equations successively in different directions, which is difficult to implement as a computational process [53]. On the contrary, we will propose a new scheme for computing the gradients of constraint $\tilde{G}_{\varepsilon, \gamma}^{i}(\tau \mid u), i \in I_{28}$ with respect to $\tau \in \mathcal{T}_{J}$ in the following theorem, the proof of which is given in Appendix B.

Theorem 5. For each $\varepsilon>0, \gamma>0$, the derivatives of the constraint functional $\tilde{G}_{\varepsilon, \gamma}^{i}(\tau \mid u), i \in I_{28}$ with respect to $\tau$ are

$$
\frac{\partial \tilde{G}_{\varepsilon, \gamma}^{i}(\tau \mid u)}{\partial \tau_{j}}=\int_{0}^{T} \frac{\partial \varphi_{\varepsilon}\left(g_{i}(x(t \mid \tau, u))\right)}{\partial g_{i}(x(t \mid \tau, u))} \frac{\partial g_{i}(x(t \mid \tau, u))}{\partial x(t \mid \tau, u)} \varphi_{j}(t \mid \tau, u) d t, i \in I_{28}, j=3,6,7,8,
$$

where $\varphi_{j}(t \mid \tau, u) \in \mathbb{R}^{14 \times 1}$, is the solution of the following auxiliary delay-differential system:

$$
\dot{\varphi}_{j}(t \mid \tau, u)=\frac{\partial f\left(x(t \mid \tau, u), x_{\tau}, u\right)}{\partial x(t \mid \tau, u)} \varphi_{j}(t \mid \tau, u)+\frac{\partial f\left(x(t \mid \tau, u), x_{\tau}, u\right)}{\partial x\left(t-\tau_{j} \mid \tau, u\right)}\left[\varphi_{j}\left(t-\tau_{j} \mid \tau, u\right)-\chi\left(t-\tau_{j}\right)\right], \forall t \in(0, T], j=3,6,7,8
$$

with

$$
\varphi_{j}(t \mid \tau, u)=\mathbf{0} \in \mathbb{R}^{14 \times 1}, \quad \forall t \in[-\bar{\tau}, 0], j=3,6,7,8 .
$$

\subsection{A parallel filled function algorithm}

Since systems in the form of (1) and (4) are often highly nonlinear and exhibit unstable behavior, it is usually not possible to derive an analytical solution to Problem TDOP. For this, the gradient-based algorithms [54] can be used to address the issue. However, such techniques are only designed to find local (rather than global) optimal solutions.

The main difficulty for global optimization is to escape from the current local minimizer and to find a better one. One of the most efficient methods to handle this issue is the filled function method which was pioneered in [55]. The generic framework of the filled function method can be described as follows [56]: (i) — An arbitrary point is taken as an initial point to minimize the objective function by using a local optimization method, and a minimizer of the objective function is obtained; (ii) - Based on the current minimizer of the objective function, a filled function is designed and a point near the current minimizer is used as an initial point to further minimize the filled function. 
As a result, a minimizer of the filled function will be found. This minimizer falls into a better region (called the basin) of the original objective function; (iii) - The minimizer of the filled function obtained in (ii) is taken as an initial point to minimize the objective function and a better minimizer of the objective function will be found; and (iv) - By repeating (ii) and (iii), the number of local minimizers will be gradually reduced, and a global minimizer will eventually be found. To overcome these shortcomings stated in [55], such as more than one parameter needing to be controlled, sensitivity to the parameters and ill-conditioning, a new filled function method is proposed [57].

Due to the highly complex nature of Problem TDOP, we propose a parallel filled function method. On each processor, we can construct a filled function. Before the next iteration, on the root processor, we can obtain the optimal time-delay vector $\tau^{*}$ of the current iteration and use it to update the current $\tau$ on each processor. Then, on each processor, we can sequentially compute until the algorithm has a successful exit [58].

However, what we need to solve is an optimization problem with both parameter bounds and continuous state constraints, to which the filled function method cannot be applied directly. To handle such constraints, we introduce the gradients of the constraint functions with respect to $\tau$ into our algorithm (see Theorem 5). With this in mind, given $\varepsilon>0, \gamma>0$, we propose a parallel filled function method, the main steps of which is stated as follows, based on Theorem 5, for solving Problem TDOP $_{\varepsilon, \gamma}$.

The parameters in algorithm 1 are defined below.

- $s$ is the slave processor index.

- $\tilde{N}$ is the number of slave processors.

- $k$ and $\ell$ are all the iteration indexes.

- $M_{K}$ is the maximal iteration of $k$.

- $\ell^{*}$ is the maximal iteration of $\ell$.

- $M_{\varrho}$ is an integer for testing convergence (if the optimal objective value has not changed after $M_{\varrho}$ iterations, then we terminate the algorithm).

- $\sigma_{1}$ is the convergence tolerance of a quality constraint for ensuring an acceptable level of the time-delay system better fits the given experimental data.

- $\sigma_{2}$ is the convergence tolerance of a cost sensitivity constraint for ensuring an acceptable level of the system performance is achieved.

- $\bar{\delta}$ is the convergence tolerance for the relative deviation of $\tau_{j}, j=3,6,7,8$.

- $\varrho_{1}$ is the convergence tolerance of the cost function.

- $\tau_{g}^{k}$ is the best time-delays found at the end of the $k$ th iteration.

- $J\left(\tau_{g}^{k}\right)$ is the best objective value found by any member of the slave processor set at the end of the $k$ th iteration.

- $\tau_{s}^{k}$ is the time-delays found by the $s$ th member of the slave processor set at the end of the $k$ th iteration.

- $J\left(\tau_{s}^{k}\right)$ is the objective value found by the $s$ th member of the slave processor set at the end of the $k$ th iteration.

- $P$ is a control parameter.

- $P^{*}$ is an upper bound of $P$.

- $\rho$ is a constant.

- $\beta$ is a small constant.

- $d_{i}, i=1, \ldots, 28$, are some directions.

- $\mathbf{h}\left(\tau_{s}^{k, j}\right)$ is the search direction of $\tau_{s}^{k, j}$.

- $\varpi\left(\tau_{s}^{k, j}\right)$ is the step-size selected by Armijo line search.

- $w$ is a given weight.

\section{Algorithm 1: \\ Master processor (Processor 0)}


Step 1 : Initialize:

- Step 1.1 : Read known data such as $\sigma_{1}, \sigma_{2}, \varepsilon, \gamma, \varrho_{1}, M_{\varrho}, \tilde{N}$ and $M_{T}$.

- Step 1.2 : Read experimental data such as $x_{0}, T, \mathcal{T}, W$.

- Step 1.3 : Broadcast (MPI_Broad) data on master processor to all slave processors.

Step 2 : Update global information

- Step 2.1 : Set $k:=1$.

- Step 2.2 : If $\left(k \geq M_{K}\right)$ or $\left(k \geq M_{\varrho}\right.$ and $\left.\left|J_{\text {best }}^{k-M_{\varrho}}-J_{\text {best }}^{k}\right|<\varrho_{1}\right)$, then goto Step 3; otherwise, goto Step 2.3.

- Step 2.3 : Receive (MPI_Recv) $J\left(\tau_{s}^{k}\right), \tau_{s}^{k}$ from the $s$ th processor and Barrier (MPI_Barrier). Compute

$$
J\left(\tau_{g}^{k}\right):=\min _{s=1, \ldots, \tilde{N}} J\left(\tau_{s}^{k}\right), \quad \tau_{g}^{k}:=\arg \min _{s=1, \ldots, \tilde{N}} J\left(\tau_{s}^{k}\right)
$$

Then, broadcast (MPI_Broad) $\tau_{g}^{k}$ to all slave processors, set $J_{\text {best }}^{k}:=J\left(\tau_{g}^{k}\right), k:=k+1$ and goto Step 2.2.

Step 3 : Report results and the algorithm has a successful exit.

Remark 3. In Step 2.2, the algorithm stops when any of the following conditions holds:

- The maximal iteration $M_{T}$ is reached;

- The deviation between the best fitness of current iteration and that of the $M_{\varrho}$ th previous iteration is less than $\varrho_{1}$.

Slave processors (Processor $s, s=1,2, \ldots, \tilde{N})$

Step 1 : Initialize: set $\mathcal{J}:=10^{6}, P^{*}:=10^{6}, \rho:=10$.

Step 2 : Update slave processor information

- Step 2.1 : Choose $\tau_{s}^{1} \in \mathcal{T}, k:=1, w:=0.5, \ell^{*}:=28$ and some directions

$$
\left\{\begin{array}{l}
d_{i}=(0, \ldots, 1, \ldots, 0), 1 \text { is at the } i \text {-th element of } d_{i}, i \in I_{14} \\
d_{i+14}=-d_{i}, i \in I_{14}
\end{array}\right.
$$

- Step 2.2 : Set $P:=1, \hbar_{1}:=0, \hbar_{2}:=0$. If $k>M_{K}$, then goto Step 3; otherwise, goto Step 2.3.

- Step 2.3 : Check and adjust.

1. Based on formulas (15), (29) and (34), check the values of $S R E\left(\tau_{s}^{k} \mid u\right),\left[\frac{\partial J\left(\tau_{s}^{k} \mid u\right)}{\partial u}\right]\left[\frac{\partial J\left(\tau_{s}^{k} \mid u\right)}{\partial u}\right]^{\mathrm{T}}$ and $\tilde{G}_{\varepsilon, \gamma}^{j}\left(\tau_{s}^{k} \mid u\right), j \in$ $I_{28}$.

2. If $\operatorname{SRE}\left(\tau_{s}^{k} \mid u\right)>\sigma_{1},\left[\frac{\partial J\left(\tau_{s}^{k} \mid u\right)}{\partial u}\right]\left[\frac{\partial J\left(\tau_{s}^{k} \mid u\right)}{\partial u}\right]^{\mathrm{T}}>\sigma_{2}$ or $\exists j \in I_{28}$ such that $\tilde{G}_{\varepsilon, \gamma}^{j}\left(\tau_{s}^{k} \mid u\right)<0$ (i.e., $\tau_{s}^{k}$ is infeasible for Problem $\mathbf{T D O P}_{\varepsilon, \gamma}$ ), then perform the first subprocedure of Algorithm 1 in [17], a gradient-descend search (minimizing $\left[\frac{\partial J\left(\tau_{s}^{k} \mid u\right)}{\partial u}\right]\left[\frac{\partial J\left(\tau_{s}^{k} \mid u\right)}{\partial u}\right]^{\mathrm{T}}$ ) or a gradient-ascent search based on formula (35) (maximizing $\left.\tilde{G}_{\varepsilon, \gamma}^{j}\left(\tau_{s}^{k} \mid u\right)\right)$ to obtain a feasible point $\breve{\tau}_{s}^{k}$ for Problem $\mathbf{T D O P}_{\varepsilon, \gamma}$ (i.e., $S R E\left(\tau_{s}^{k} \mid u\right) \leq \sigma_{1},\left[\frac{\partial J\left(\breve{\tau}_{s}^{k} \mid u\right)}{\partial u}\right]\left[\frac{\partial J\left(\breve{\tau}_{s}^{k} \mid u\right)}{\partial u}\right]^{\mathrm{T}} \leq$ $\sigma_{2}$ and $\left.\forall j \in I_{10}, \tilde{G}_{\varepsilon, \gamma}^{j}\left(\breve{\tau}_{s}^{k} \mid u\right) \geq 0\right)$ and set $\breve{\tau}_{s}^{k} \rightarrow \tau_{s}^{k}$.

$$
\text { If } \begin{cases}\hbar_{2}=0, & \left\{\begin{array}{l}
\hbar_{1}=0, \\
\hbar_{1}=1,
\end{array}\right. \\
\hbar_{2}=1, & \text { then goto Step 2.4, }\end{cases}
$$


- Step 2.4 Use Hooke-Jeeves algorithm with initial value $\tau_{s}^{k}$ to obtain local optimal solution of Problem TDOP $\mathbf{P}_{\varepsilon, \gamma}$, still denoted by itself $\tau_{s}^{k}$, set $\hbar_{1}=1$ and goto Step 2.3.

- Step 2.5 : Based on [57], construct

$$
\widetilde{F}\left(\tau, \tau_{s}^{k}, P\right):=\frac{P}{1+\left\|\tau-\tau_{s}^{k}\right\|} \Gamma_{\frac{1}{P}}\left(J(\tau \mid u)-J\left(\tau_{s}^{k} \mid u\right)\right), \tau \in \mathcal{T}
$$

with

$$
\Gamma_{\frac{1}{P}}\left(J(\tau \mid u)-J\left(\tau_{s}^{k} \mid u\right)\right)= \begin{cases}\frac{1}{P}, & \text { if } J(\tau \mid u)-J\left(\tau_{s}^{k} \mid u\right) \geq 0, \\ {\left[J(\tau \mid u)-J\left(\tau_{s}^{k} \mid u\right)\right]^{3}+\frac{1}{P},} & \text { if } J(\tau \mid u)-J\left(\tau_{s}^{k} \mid u\right)<0,\end{cases}
$$

and $\operatorname{set} \ell:=1$.

- Step 2.6 : If $\ell>\ell^{*}$, then goto Step 2.7; otherwise, set $\tau:=\tau_{s}^{k}+\beta d_{\ell}$ and goto Step 2.8.

- Step 2.7 : If $P<P^{*}$, then increase $P$ by $P:=\rho P$ and goto Step 2.5 ; otherwise, $\tau_{s}^{k}$ is taken as a global minimizer of $J\left(\tau_{s}^{k} \mid u\right)$, set $\hbar_{2}:=1$ and goto Step 2.3 .

- Step 2.8 : Use $\tau$ as the initial point to minimize $\widetilde{F}\left(\tau, \tau_{s}^{k}, P\right)$ and denote the sequence point generated by a local optimization algorithm as $\tau_{J}, J=1,2, \cdots$. If $\exists_{J} \in\{1,2, \cdots\}$ such that $\tau_{J} \notin \mathcal{T}$, set $\ell:=\ell+1$ and goto Step 2.6; otherwise, find a minimizer $\tau^{\prime} \in \mathcal{T}$ of $\widetilde{F}\left(\tau, \tau_{s}^{k}, P\right)$. If $J\left(\tau^{\prime} \mid u\right)<J\left(\tau_{s}^{k} \mid u\right)$, then set $\tau_{s}^{k}:=\tau^{\prime}$ and goto Step 2.5; otherwise goto Step 2.7 .

- Step 2.9 : Send (MPI_Send) $J\left(\tau_{s}^{k} u\right)$ and $\tau_{s}^{k}$ to master processor.

- Step 2.10 : Based on broadcasted $\tau_{g}^{k}$ in master processor, obtain $\tau_{g}^{k}$. Then, use $\tau_{g}^{k}$ to replace $\tau_{s}^{k+1}$, set $k:=k+1$. and goto Step 2.2.

Step 3 : The algorithm has a abnormal exit.

Remark 4. In Step 2.6, $\beta$ needs to be selected carefully. A large $\beta$ may cause losing the better solution of the original problem, while a small $\beta$ may cause the local optimization to fail to progress in the minimization of $\widetilde{F}\left(\tau, \tau_{s}^{k}, P\right)$. In our algorithm, $\beta$ is selected to guarantee that $\left\|\nabla \widetilde{F}\left(\tau, \tau_{s}^{k}, P\right)\right\|$ is greater than a threshold (e.g. take $\beta$ as $10^{-3}$ ). For specific problems, the selection of $\beta$ is related to the number of minimizers of the objective function and the size of the feasible region. The fewer minimizers of the objective function and the larger size of the feasible region, the larger $\beta$ should be [57].

By Algorithm 1, we can develop the following algorithm to solve Problem TDOP.

\section{Algorithm 2}

Step 1: Choose initial values of $\varepsilon>0, \gamma>0$.

Step 2 : Solve Problem TDOP $_{\varepsilon, \gamma}$ using Algorithm 1 to give $\tau_{\varepsilon, \gamma}^{*}$.

Step 3 : Check feasibility of $g_{j}\left(x\left(t \mid \tau_{\varepsilon, \gamma}^{*}, u\right)\right) \geq 0$ for $\forall t \in[0, T], j \in I_{28}$.

Step 4 : If $\tau_{\varepsilon, \gamma}$ is feasible, goto Step 5; otherwise, set $\gamma:=\alpha_{1} \gamma$. If $\gamma>\bar{\gamma}$, where $\bar{\gamma}$ is a prespecified positive constant, goto Step 2; otherwise goto Step 6.

Step 5 : Set $\varepsilon:=\alpha_{2} \varepsilon$. If $\varepsilon>\bar{\varepsilon}$, where $\bar{\varepsilon}$ is a prespecified positive constant, goto Step 2; otherwise goto Step 6 .

Step 6 : Output $\tau_{\varepsilon, \gamma}^{*}$ and stop.

Remark 5. In Algorithm 2, $\varepsilon$ is a parameter controlling the accuracy of the smoothing approximation; $\gamma$ is a parameter controlling the feasibility of the constraint (6); the parameters $\alpha_{1}$ and $\alpha_{2}$ must be chosen to be less than 1. 
Remark 6. Because computational efficiency is a crucial issue, we need to consider the accuracy of optimal solution of the system (1) and the question of computational time with regard to $\epsilon, \gamma$ in Algorithm 2. Clearly, the low accuracy of optimal solution of the system (1) and long runtime are sufficient for a large $\epsilon, \gamma$. The accuracy of optimal solution of the system (1) may be improved and runtime is long as $(\epsilon, \gamma)$ approaches to $(0,0)$. With this in mind, $\bar{\varepsilon}$ and $\bar{\gamma}$ are two predefined parameters ensuring the termination of the algorithm.

Remark 7. Clearly, we cannot check the feasibility of $g_{j}\left(x\left(t \mid \tau_{\varepsilon, \gamma}^{*}, u\right)\right) \geq 0$, for every $t \in[0, T]$. In practice, we choose a set which contains a dense enough set of points in $[0, T]$. Check the feasibility of $g_{j}\left(x\left(t \mid \tau_{\varepsilon, \gamma}^{*}, u\right)\right) \geq 0$, over this set for each $j=1, \ldots, 28$.

Remark 8. In (20), the formula $E_{j}\left(t \mid S R D_{j}, \tau, u\right), j=6, \ldots, 14$, is an integral form, which would be difficult to compute directly. Hence, our method is to divide the perturbation space with uniformity into $\mathcal{K}_{0}$ subdivisions. The formula $E_{j}\left(t \mid S R D_{j}, \tau, u\right), j=6, \ldots, 14$, becomes

$$
E_{j}^{\mathcal{K}_{0}}(t \mid \tau):=\frac{\sum_{\ell=1}^{\mathcal{K}_{0}} S R D_{j}\left(x\left(t \mid \tau^{\ell}, u\right), x(t \mid \tau, u)\right)}{\mathcal{K}_{0}}, j=6, \ldots, 14,
$$

where $\tau^{\ell}, \ell \in I_{\mathcal{K}_{0}}$, are uniformly distributed in field of $B(\tau, \bar{\delta}), \tau \in \mathcal{T}_{J}$ and $\bar{\delta}$ is the radius of this field.

To find a appropriate value of the number of subdivisions $\mathcal{K}_{0}$, we propose the following algorithm:

\section{Algorithm 3}

Step 1: Given $\bar{\epsilon}_{1}>0$, choose two positive integers $\mathcal{M}_{0}<\mathcal{N}_{0}$, let $\mathcal{M}:=\mathcal{M}_{0}, \mathcal{N}:=\mathcal{N}_{0}$ and set $\bar{\varrho}:=0$.

Step 2: It follows from (38) that we compute the value of $E_{j}^{\mathcal{M}}(\cdot \mid \tau)$ and $E_{j}^{\mathcal{N}}(\cdot \mid \tau)$.

Step 3 : If $\left|\frac{\sum_{j=6}^{14} \int_{0}^{T}\left[E_{j}^{\mathcal{M}}(t \mid \tau)-E_{j}^{\mathcal{N}}(t \mid \tau)\right] d t}{\sum_{j=6}^{14} \int_{0}^{T} E_{j}^{\mathcal{N}}(t \mid \tau) d t}\right| \leq \bar{\epsilon}_{1}$ then set $\mathcal{N}:=\mathcal{M}, \mathcal{M}:=\lfloor 0.5 \mathcal{M}\rfloor, \bar{\varrho}:=1$ and goto Step 2; otherwise, goto Step 4.

Step 4: If $\left|\frac{\sum_{j=6}^{14} \int_{0}^{T}\left[E_{j}^{\mathcal{M}}(t \mid \tau)-E_{j}^{\mathcal{N}}(t \mid \tau)\right] d t}{\sum_{j=6}^{14} \int_{0}^{T} E_{j}^{\mathcal{N}}(t \mid \tau) d t}\right| \leq \bar{\epsilon}_{1}$ and $\bar{\varrho}=0$, then set $\mathcal{M}:=\lfloor 2 \mathcal{M}\rfloor, \mathcal{N}:=\lfloor 2 \mathcal{N}\rfloor$ and goto Step 2; otherwise, goto Step 5.

Step 5: If $\bar{\varrho}=1$, then set $\mathcal{K}_{0}:=\lfloor 2 \mathcal{M}\rfloor$, output $\mathcal{K}_{0}$ and stop.

\section{Numerical results}

Objectives: We attempt to determine optimal values of time-delays by solving Problem TDOP through the application of Algorithms 1-2.

To solve Problem TDOP, some known data is stated as follows: in Problem TDOP, $M:=10^{4}, T:=6.92 ; x_{0}:=$ $[0.102,418.2609,0,0,0,0,0,0,0,0,0,0,0,0]^{\mathrm{T}}$; the system (1) is solved by using Euler method with a step size of $1 / 72000(\mathrm{~h})$. In (22), to make the expectation and variance in the same order of magnitude, we set $w:=10$, which can be seen in Figs. 3-11. In Algorithm $1, \sigma_{1}:=30 \%, \sigma_{2}:=20, \bar{\delta}:=0.1, \varrho_{1}:=10^{-3}, M_{\varrho}:=10^{2}, M_{K}:=10^{4}, \tilde{N}:=$ $64, \hat{\tau}_{3}=\hat{\tau}_{6}=\hat{\tau}_{7}=\hat{\tau}_{8}:=1$. In Algorithm 2, $\varepsilon:=0.1, \gamma:=0.01, \alpha_{1}:=0.1, \alpha_{2}:=0.01, \bar{\varepsilon}:=1.0 \times 10^{-7}, \bar{\gamma}:=1.0 \times 10^{-7}$. In Algorithm $3, \bar{\epsilon}_{1}:=0.1, \mathcal{M}:=10^{2}, \mathcal{N}:=10^{5}$. The initial function $\phi(t), t \in[-\bar{\tau}, 0)$ is obtained by the cubic spline interpolation [61] of the measured data at the measurement time point priori to the zero time point. 
Algorithm 3 is called to seek for the appropriate value of the number of subdivisions $\mathcal{K}_{0}$. According to (21), (22) and (38), we calculate 1000 values of the cost function for $\mathcal{K}_{0}=1 \times 10^{3}, 2 \times 10^{3}, \ldots, 1 \times 10^{4}$. Our results are shown as box plots in Fig. 1. Note that, as expected, the results for $\mathcal{K}_{0} \geq 7000$ show far less variation in the value of the cost function than the results for $\mathcal{K}_{0}<7000$. That is, we can see that 7000 is a appropriate value of $\mathcal{K}_{0}$.

Problem TDOP is solved by using Algorithms 1-2 on Lenovo DeepComp 1800 PC-cluster Server composed of 16 nodes (which is Dalian University of Technology, China). Each node is equipped with two Intel 5420 CPU (4 Core, 64-bit, clocked at $2.5 \mathrm{GHz}$ ) and $8 \mathrm{~GB}$ memory. Applying Algorithms 1-2 to Problem TDOP, we obtain optimal values of time-delays, which are shown in Table 2. The results are shown in Figs. 2-16.

For comparison, the relative errors between the computational values and the experimental data in this work and the ones in [26] are listed in Table 3, in which the relative errors are defined as

$$
e_{\ell}:=\left\{\sum_{l=1}^{\widetilde{\mathcal{N}}}\left|x_{\ell}\left(t_{l} \mid \tau^{*}, u\right)-y_{\ell}\left(t_{l}\right)\right|\right\} /\left\{\sum_{l=1}^{\widetilde{\mathcal{N}}}\left|y_{\ell}\left(t_{l}\right)\right|\right\}, t_{l}=\imath \times \frac{T}{\widetilde{\mathcal{N}}}, \widetilde{\mathcal{N}}=17, \ell=1,2,3 .
$$

The errors in this paper $e_{1}=9.81 \%, e_{2}=4.23 \%, e_{3}=8.15 \%$ and the reported results in [26] are shown in Table 3. From Table 3, we can see that the relative errors are cut down greatly in comparison with the ones in [26]. More accurately, the relative error of Biomass is down $39.52 \%$ in this paper compared with [26]; the relative error of extracellular glycerol drops by $64.57 \%$ from [26] to this paper; the relative error extracellular 1,3-PD falls $68.08 \%$ in this paper from [26]. Furthermore, the concentration changes computed by the optimal values of time-delays are plotted in Fig. 2. Note that, as expected, the curves in Fig. 2 also confirm that the nonlinear time-delay dynamical system of dha-regulon with the optimal values of time-delays can describe the batch fermentation process reasonably. For the difficulty in accurately measuring the concentration of intracellular substances, the expectation and variance of relative deviation with respect to the concentration of intracellular substances are plotted in Figs 3-11. We can see from the left two sub-figures of Figs. 3-11 that the expectation and variance of relative deviation has far less variation with respect to the concentration of intracellular substances on time-delays $\tau^{*}$ than the results in the right two sub-figures of Figs. 3-11 for time-delays $\tau^{-}$. That is, the relative deviation is less sensitive to fluctuations on time-delays $\tau^{*}$ than the results in the right two sub-figures of Figs. 3-11 for time-delays $\tau^{-}$. Therefore, Figs. 3-11 show the superiority for our system over the system in [26]. From what we have mentioned above, our system can reasonably characterize the concentration changes of both extracellular substances (biomass, extracellular glycerol, extracellular 1,3-PD, acetic acid and ethnol) and intracellular substances (intracellular glycerol, 3-HPA, intracellular 1,3-PD, $m_{R}, \mathrm{R}, \mathrm{mGDHt}, \mathrm{GDHt}, \mathrm{mPDOR}$ and PDOR).

From Figs. 12-13, the continuous inequality constraints (26) is satisfied for $t \in[0, T]$.

Phase planes among the concentrations of biomass $\left(x_{1}\right)$, extracellular glycerol $\left(x_{2}\right)$, extracellular $1,3-\mathrm{PD}\left(x_{3}\right)$ in Fig. 14. A negative correlation between the concentrations of extracellular biomass $\left(x_{1}\right)$ and extracellular glycerol $\left(x_{2}\right)$ is shown in Fig. 14 of the left and a negative correlation between the concentrations of extracellular 1,3-PD $\left(x_{3}\right)$ and extracellular glycerol $\left(x_{2}\right)$ is shown in the Fig. 14 of the right. Fig. 14 of the middle shows a positive correlation between the concentrations of biomass $\left(x_{1}\right)$ and extracellular 1,3-PD $\left(x_{3}\right)$.

It turns out that the 22 nd component of $u$, i.e., $u_{22}$, is the most critical parameter in terms of cost sensitivity: $\partial J / \partial u_{22}$ is the dominant term in the values of cost sensitivity in Fig. 15. We now test the cost sensitivity of the system (1) under optimal values of time-delays $\tau^{*}$. To do this, we perturb the nominal value of $u_{22}$ by a small amount and then simulate the system under optimal values of time-delays $\tau^{*}$. The variation in the values of cost function due to $u_{22}$ is shown in Fig. 16. The first thing to note is that the value of the cost function to Problem TDOP excluding constraint (25) is very sensitive with respect to changes in $u_{22}$. For example, a change of $4 \times 10^{-5}$ in the value of $u_{22}$ causes the value of the cost function to more than double. The change in the value of the cost function to Problem TDOP including constraint (25), however, is much smaller. The value of the cost function to Problem TDOP including constraint (25) under optimal values of time-delays $\tau^{*}$ hardly changes when $u_{22}$ is changed.

Table 2. The optimal and non-optimal values of time-delays to the system (1).

\begin{tabular}{cccccccc}
\hline$\tau_{3}^{*}$ & $\tau_{6}^{*}$ & $\tau_{7}^{*}$ & $\tau_{8}^{*}$ & $\tau_{3}^{-}$ & $\tau_{6}^{-}$ & $\tau_{7}^{-}$ & $\tau_{8}^{-}$ \\
0.704723 & 0.641289 & 0.326841 & 0.438276 & 0.5 & 0.5 & 0.5 & 0.5 \\
\hline
\end{tabular}


Table 3. The relative errors and the value of the cost sensitivity under optimal values of time-delays $\tau^{*}$.

\begin{tabular}{ccc}
\hline Substances & $e_{\ell}(\%)$ in the paper & $e_{\ell}(\%)$ in [26] \\
\hline$\ell=1$ (Biomass) & 9.81 & 16.22 \\
$\ell=2$ (extracellular Glycerol) & 4.23 & 11.94 \\
$\ell=3$ (extracellular 1,3-PD) & 8.15 & 25.53 \\
\hline
\end{tabular}

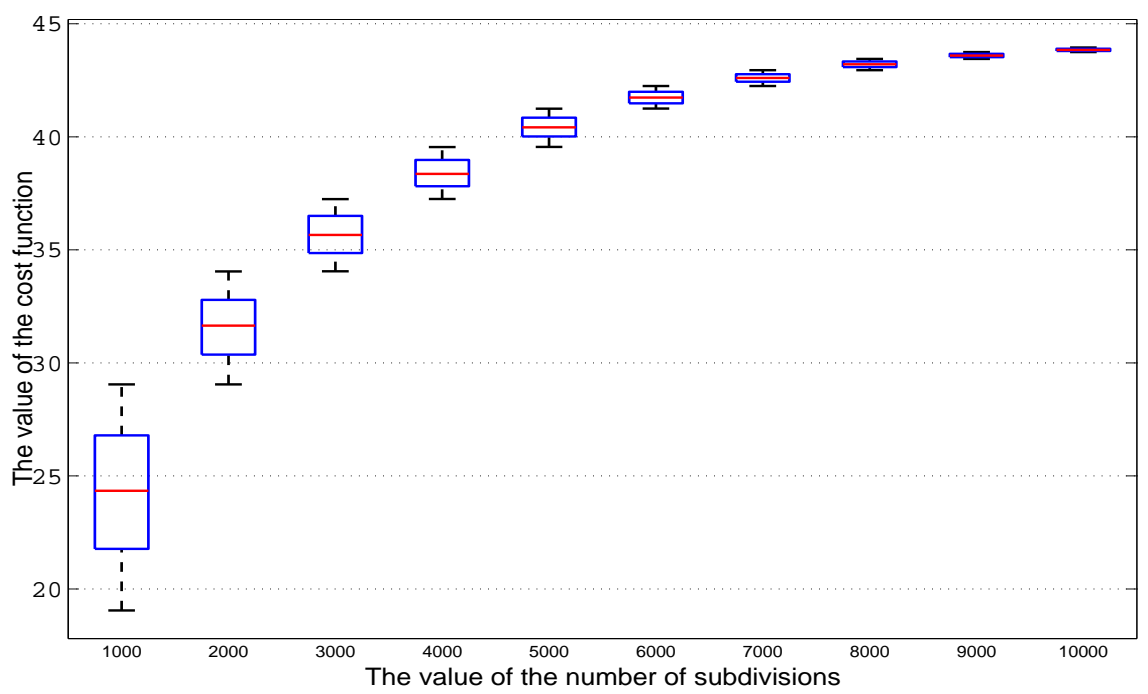

Fig. 1. The value of the cost function for the number of subdivisions by uniform distribution.

\section{Discussions and conclusions}

In the paper, we consider a nonlinear time-delay dynamical system of dha-regulon with unknown time-delays to describe the actual process of glycerol batch culture. A quantitative biological robustness for the concentrations of intracellular substances is defined by penalizing a weighted sum of the expectation and variance of the relative deviation between system outputs before and after time-delays are perturbed. We formulate a nonlinear dynamic optimization problem in which the time-delays are decision variables and the cost function is to minimize the biological robustness. This optimization problem is subject to the time-delay system, continuous state inequality constraints, parameter constraints, a quality constraint and a cost sensitivity constraint. It is approximated as a sequence of nonlinear mathematical programming sub-problems through the application of constraint transcription and local smoothing approximation techniques. A parallel algorithm is proposed to solve these nonlinear programming sub-problems. Finally, numerical simulation results verified the effectiveness of the numerical solution method.

Many delay systems that arise in applications are actually nonlinear. Our approach to delay identification is more widely applicable to nonlinear delay-differential systems modelled by ODEs. An interesting work for future research will be to study multi-stage analysis and optimal control in batch culture.

\section{Acknowledgements}

This work was supported by the National Natural Science Foundation of China (Grant Nos. 11171050 and 11371164), the National Science Foundation for the Youth of China (Grant Nos. 11201267, 11301051, 11301081, 

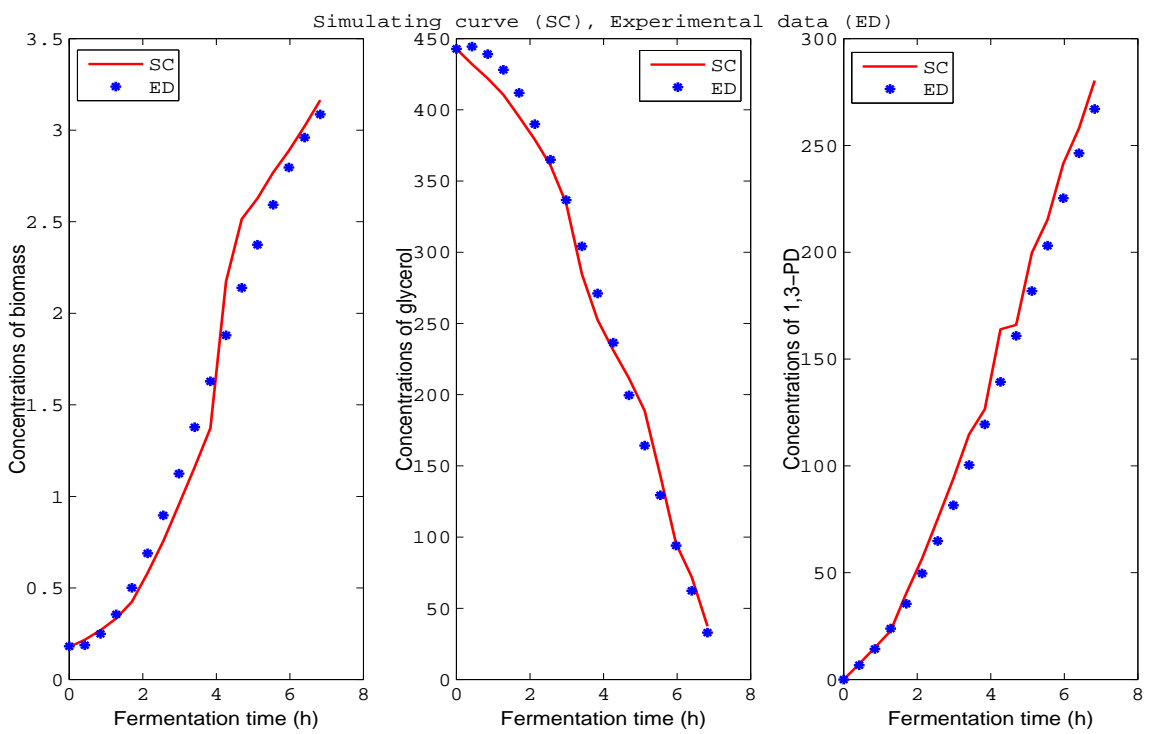

Fig. 2. Comparisons of experimental data and simulating curves of biomass, extracellular glycerol and extracellular 1,3-PD concentrations in batch culture based on the function fitted by experimental data. The horizontal axes represent time. The vertical axes represent the concentrations of biomass, extracellular glycerol and extracellular 1,3-PD concentrations.
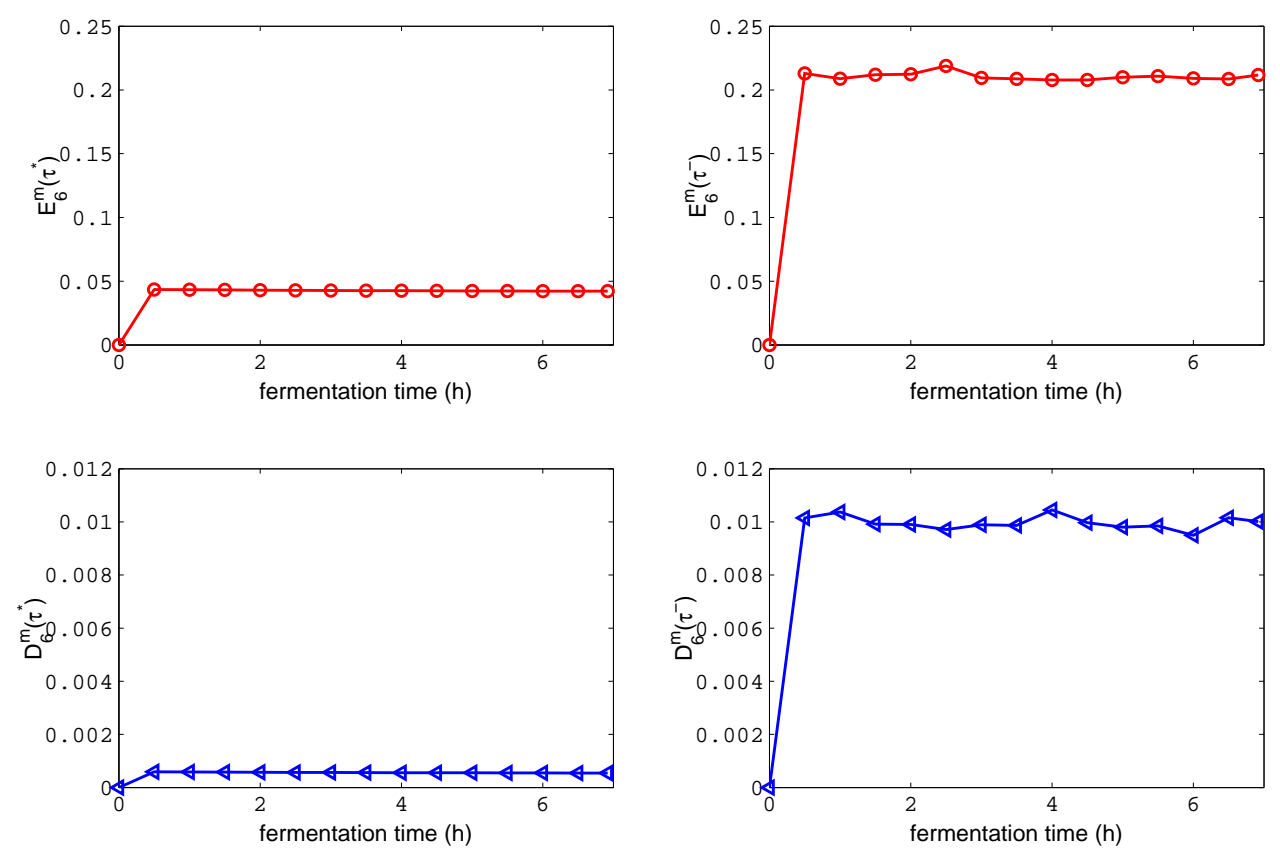

Fig. 3. The expectation and variance of relative deviation with respect to intracellular glycerol. 

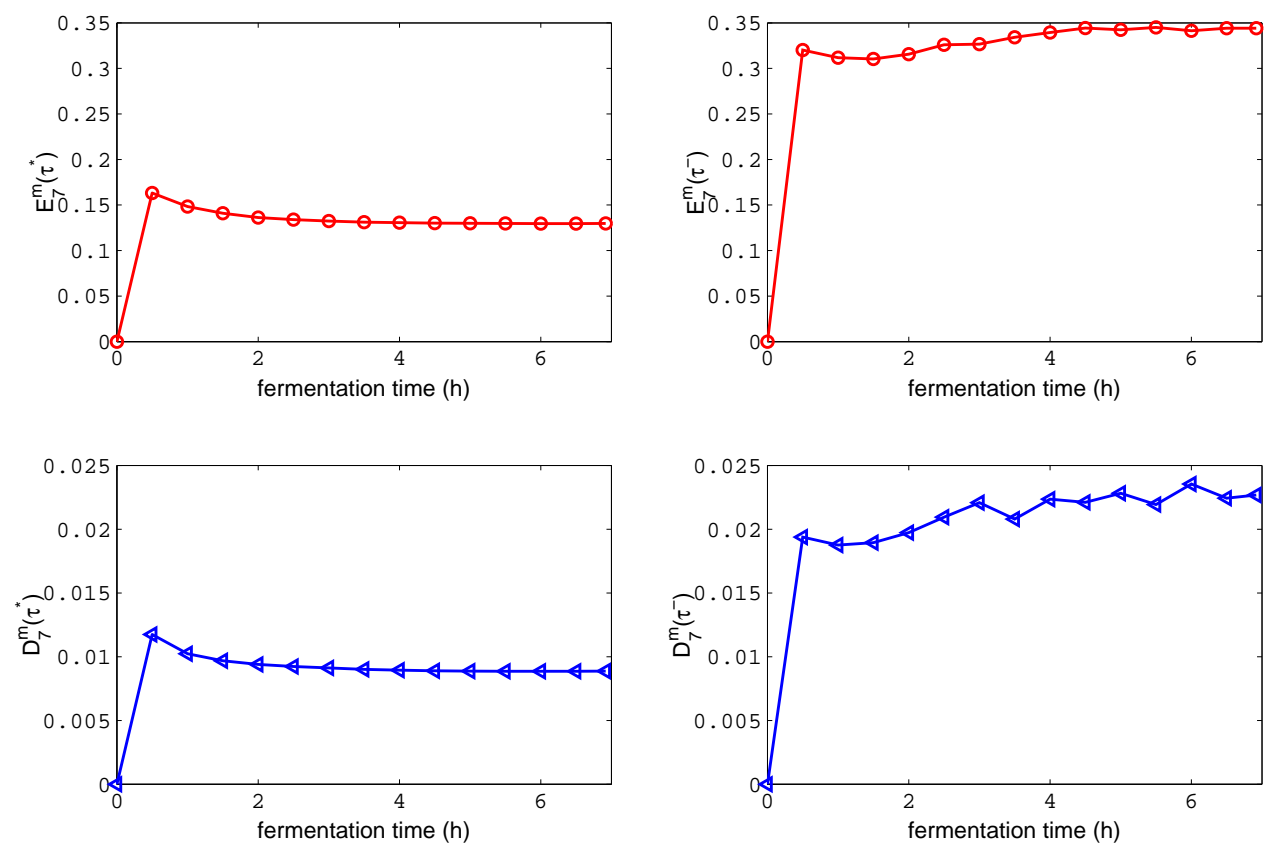

Fig. 4. The expectation and variance of relative deviation with respect to intracellular 3-HPA.
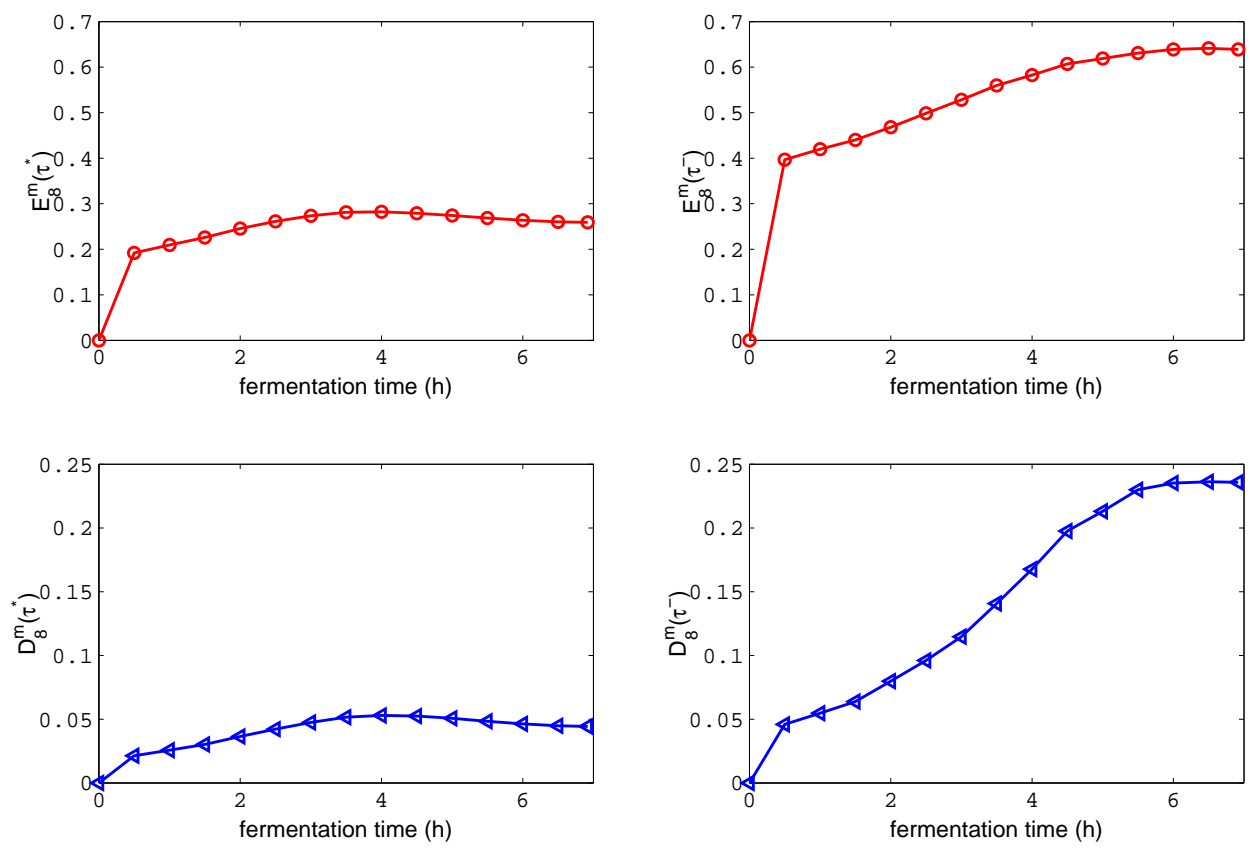

Fig. 5. The expectation and variance of relative deviation with respect to intracellular 1,3-PD. 

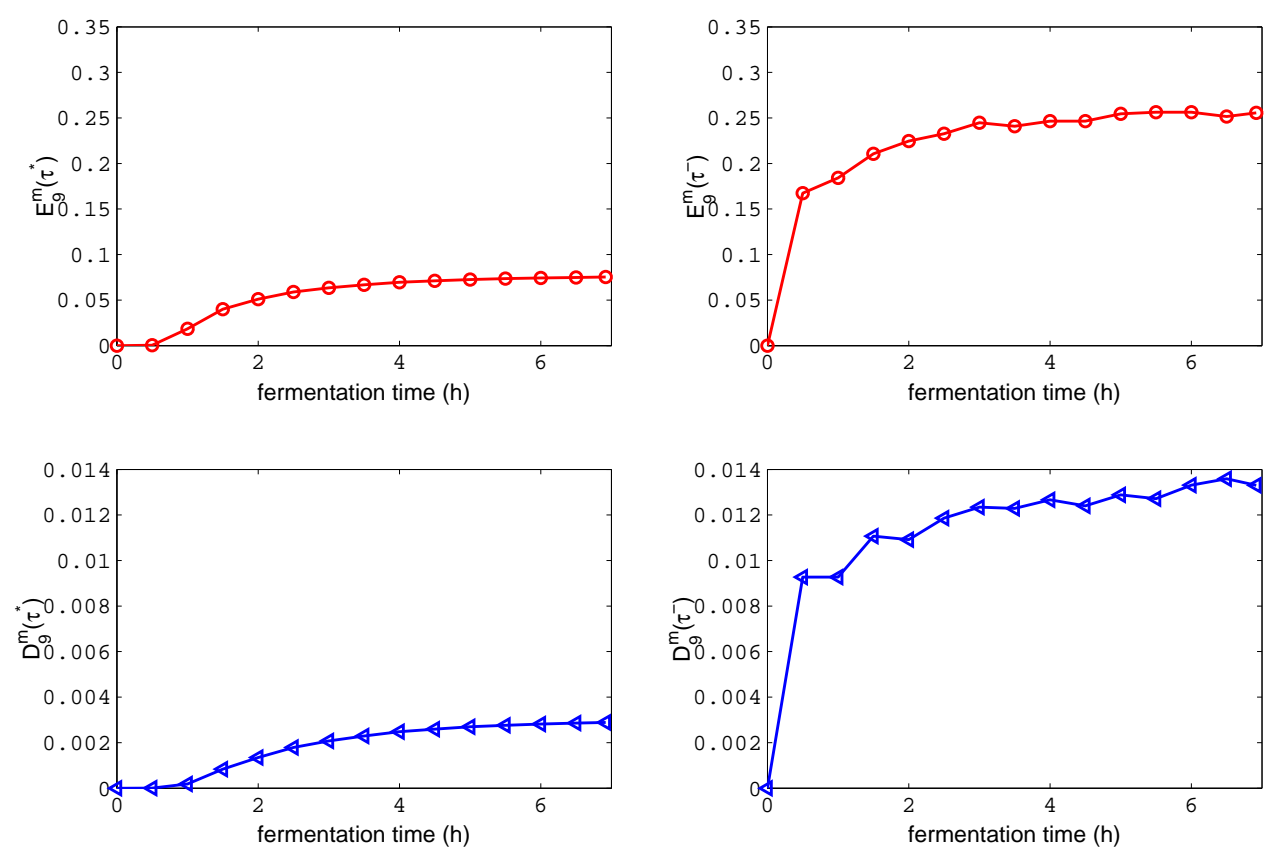

Fig. 6. The expectation and variance of relative deviation with respect to $m R$ (mRNA coding repressor).
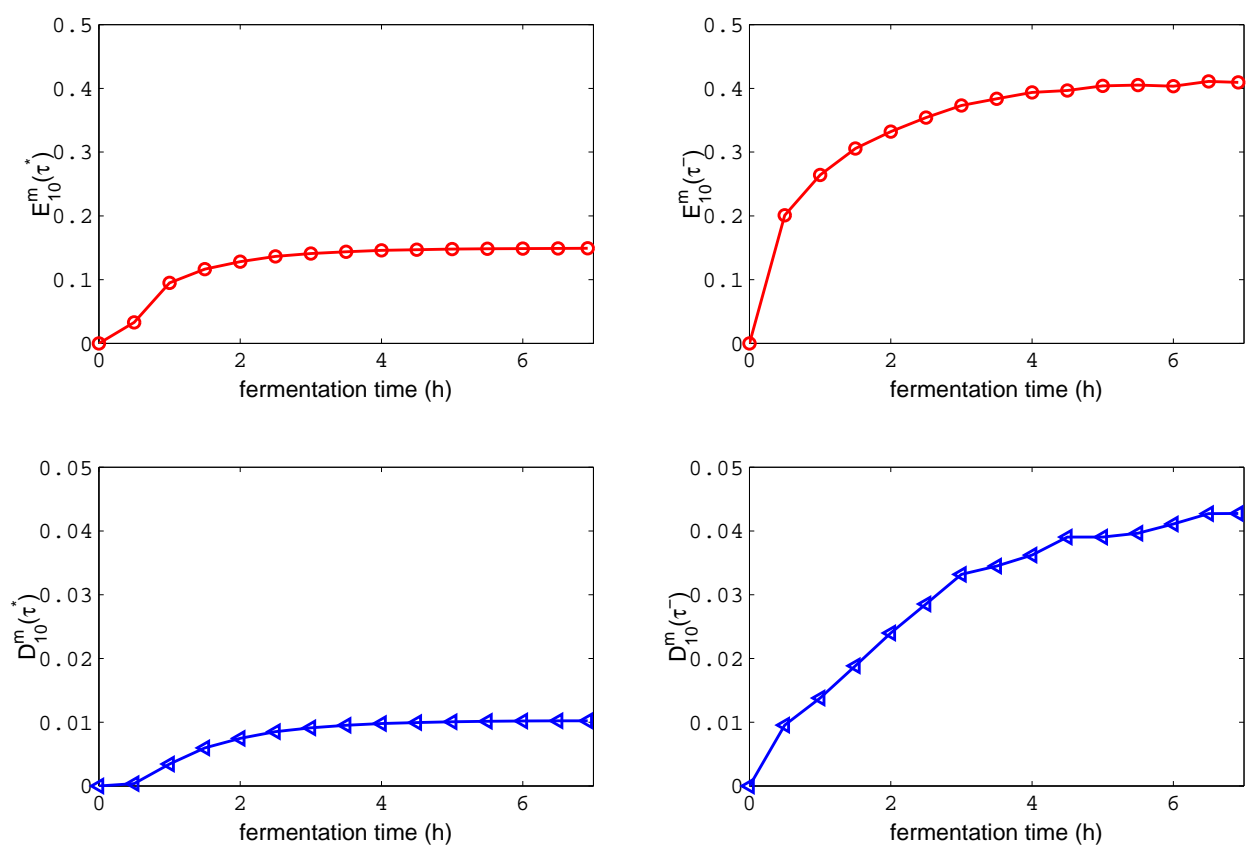

Fig. 7. The expectation and variance of relative deviation with respect to $R$ (free repressor). 

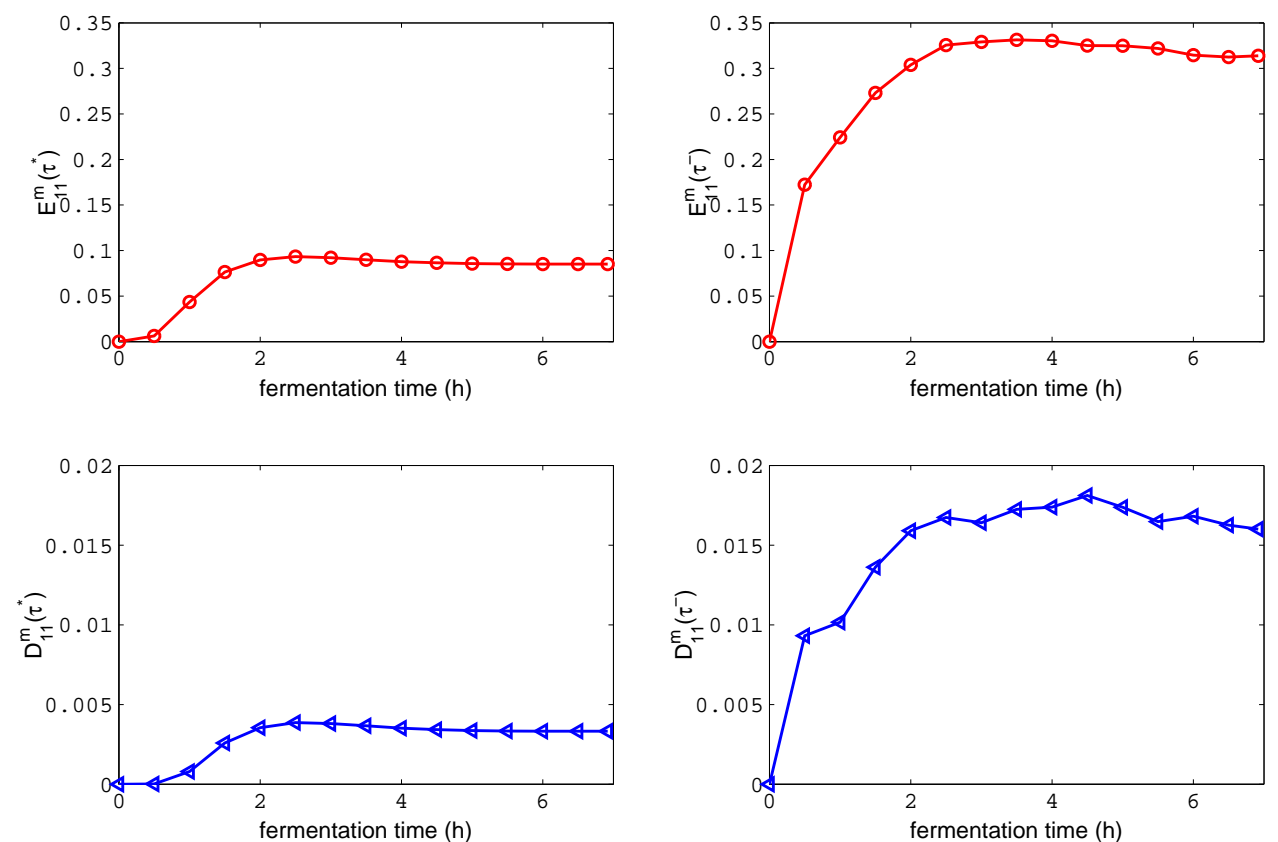

Fig. 8. The expectation and variance of relative deviation with respect to mGDHt.
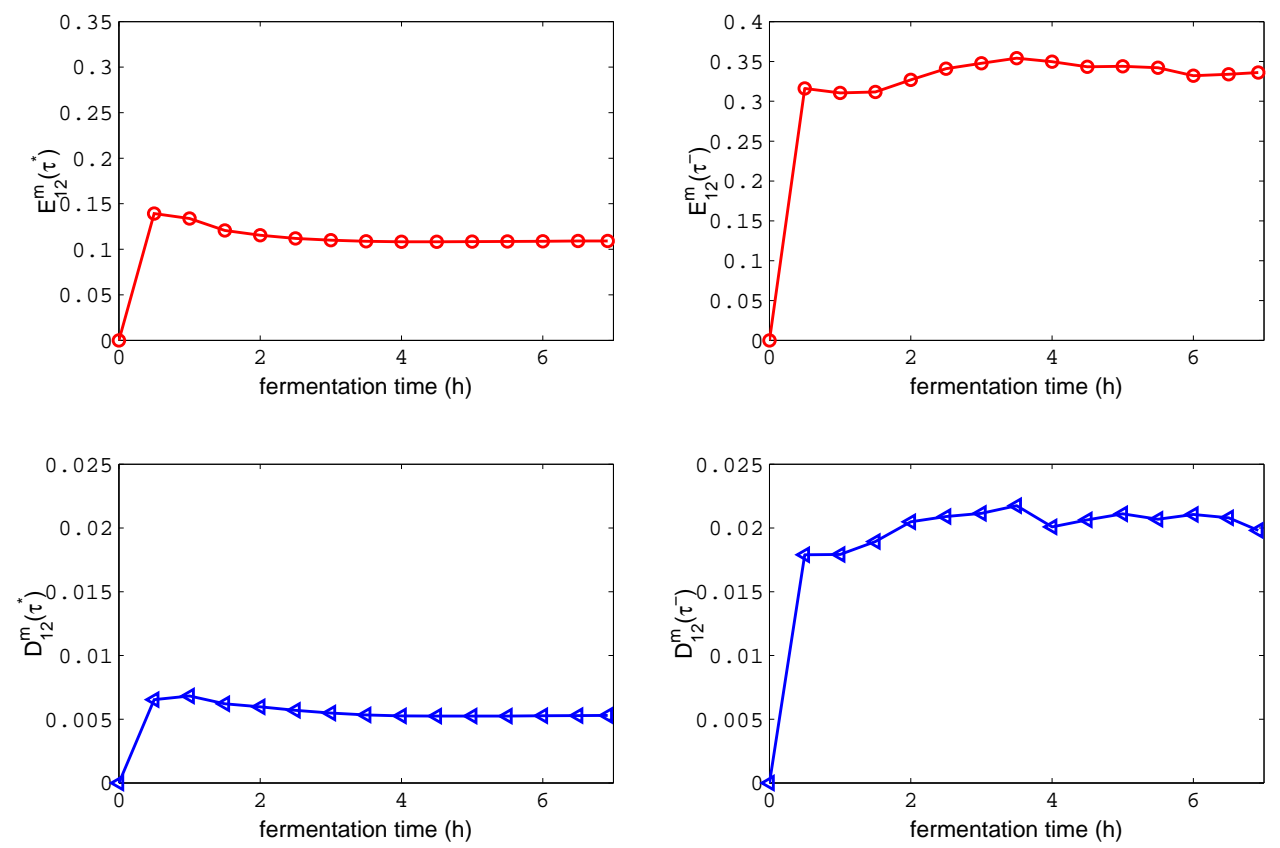

Fig. 9. The expectation and variance of relative deviation with respect to GDHt (glycerol dehydratase). 

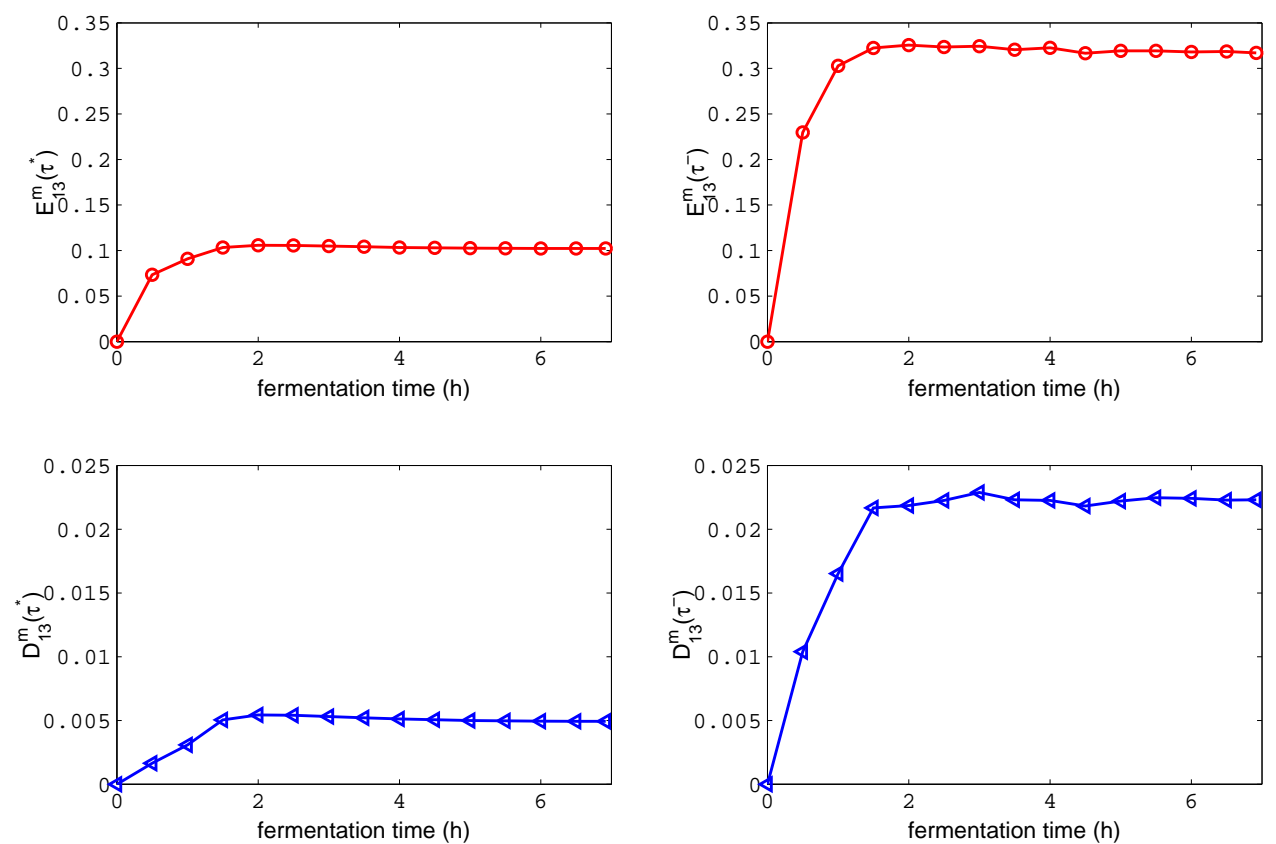

Fig. 10. The expectation and variance of relative deviation with respect to mPDOR.
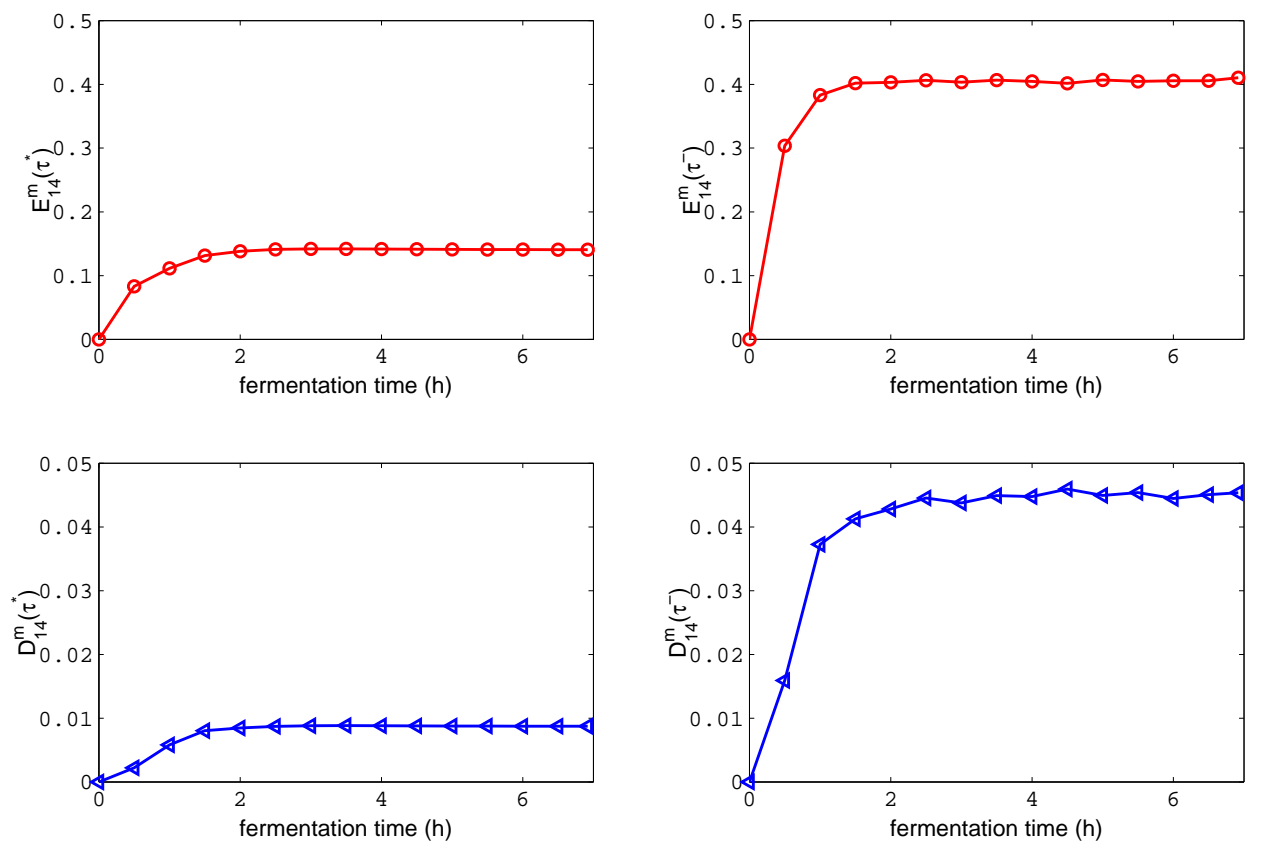

Fig. 11. The expectation and variance of relative deviation with respect to PDOR (1,3-PD oxydoreductase). 

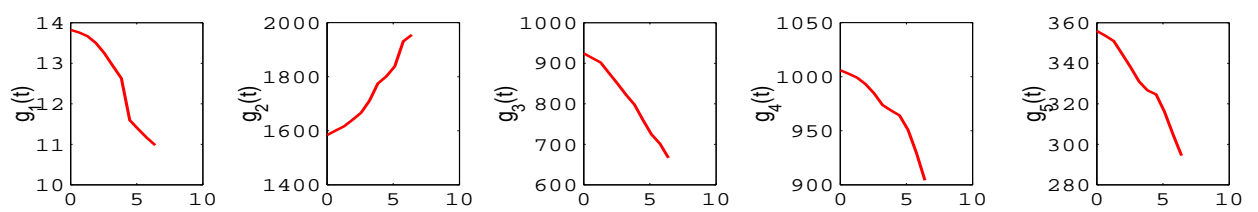

Fermentation time $\mathrm{t}(\mathrm{h})$
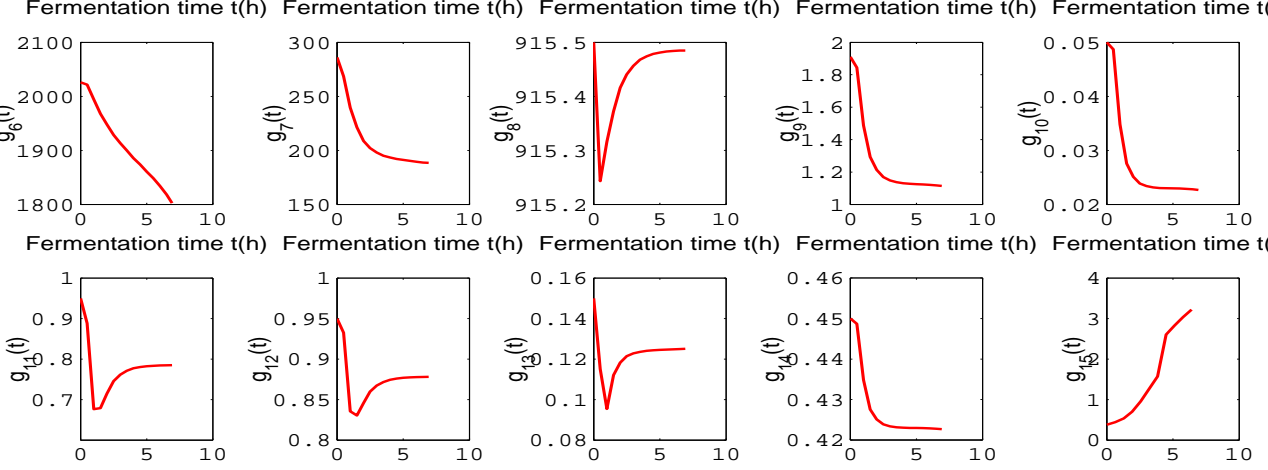

Fermentation time $\mathrm{t}(\mathrm{h})$ Fermentation time $\mathrm{t}(\mathrm{h})$ Fermentation time $\mathrm{t}(\mathrm{h})$ Fermentation time $\mathrm{t}(\mathrm{h})$ Fermentation time $\mathrm{t}(\mathrm{h})$

Fig. 12. $g_{j}(t):=g_{j}\left(x\left(t \mid \tau^{*}, u\right)\right), j=1, \ldots, 15$, under $\tau^{*}$ in batch culture process in $t \in[0, T]$.
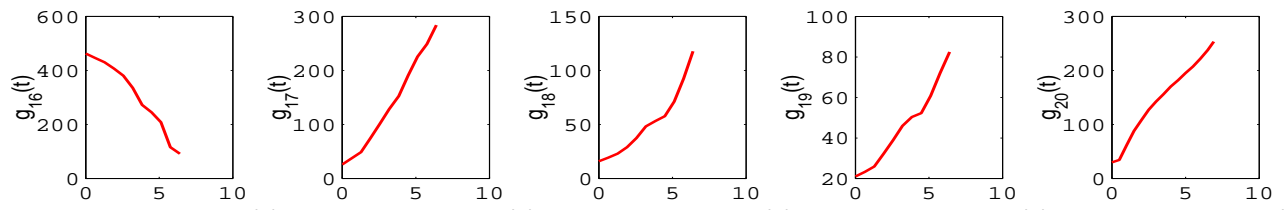

Fermentation time $t(h)$ Fermentation time $t(h)$ Fermentation time $t(h)$ Fermentation time $t(h)$ Fermentation time $t(h)$
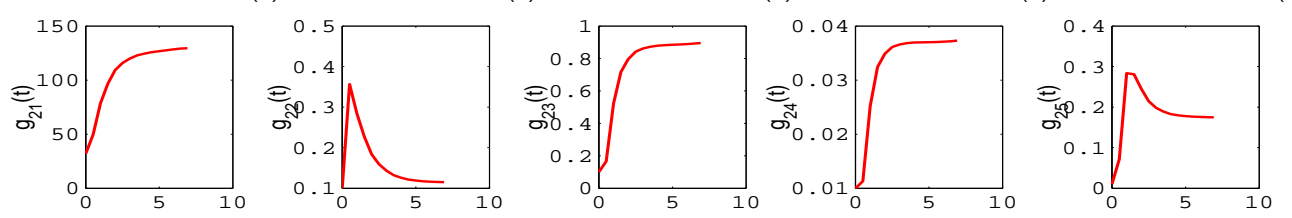

Fermentation time $\mathrm{t}(\mathrm{h})$ Fermentation time $\mathrm{t}(\mathrm{h})$
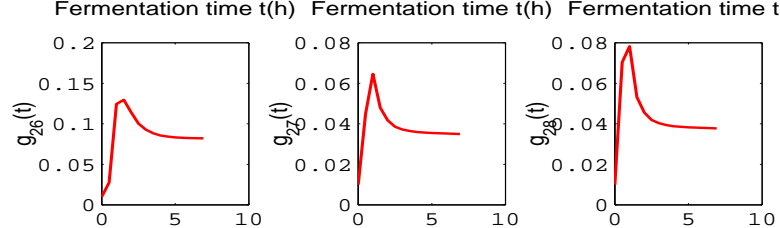

Fermentation time $t(h)$

Fermentation time $t(h)$ Fermentation time $t(h)$ Fermentation time $t(h)$

Fig. 13. $g_{j}(t):=g_{j}\left(x\left(t \mid \tau^{*}, u\right)\right), j=16, \ldots, 28$, under $\tau^{*}$ in batch culture process in $t \in[0, T]$. 

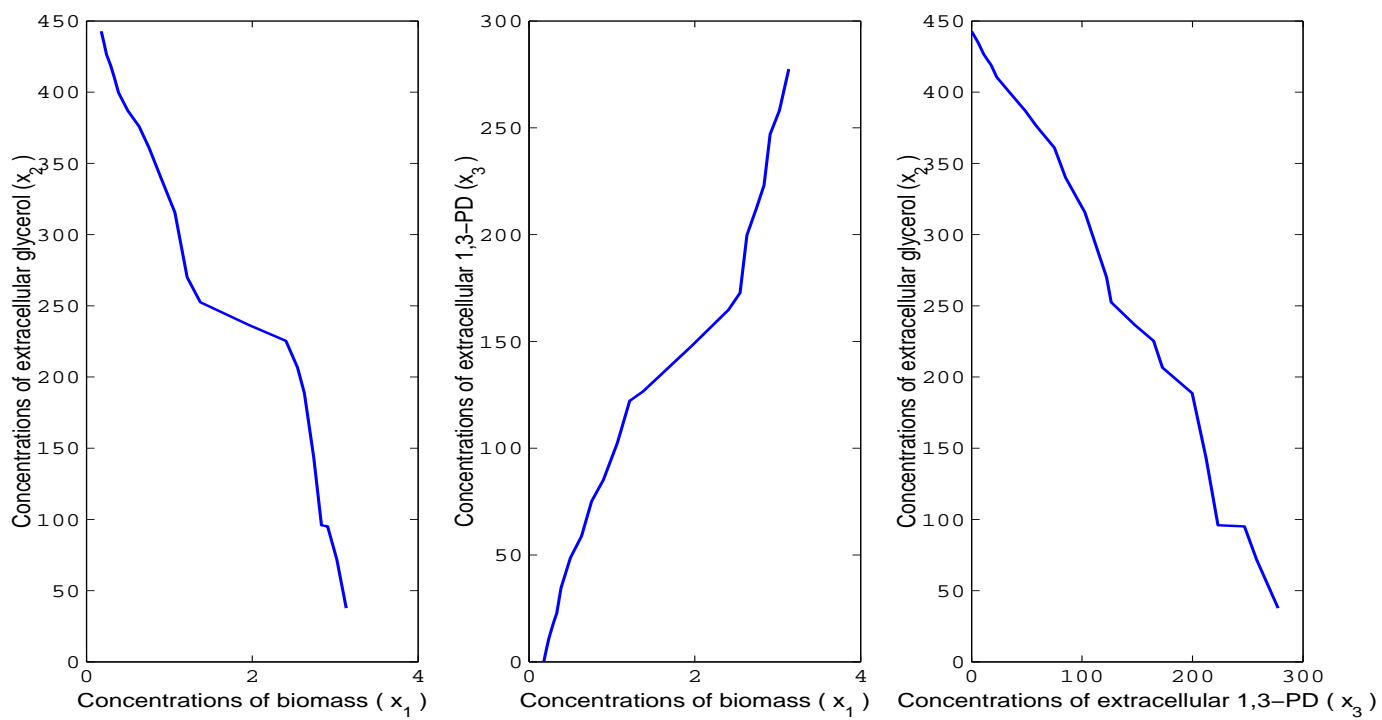

Fig. 14. Phase planes among the concentrations of biomass $\left(x_{1}\right)$, extracellular glycerol $\left(x_{2}\right)$, extracellular 1,3-PD $\left(x_{3}\right)$.

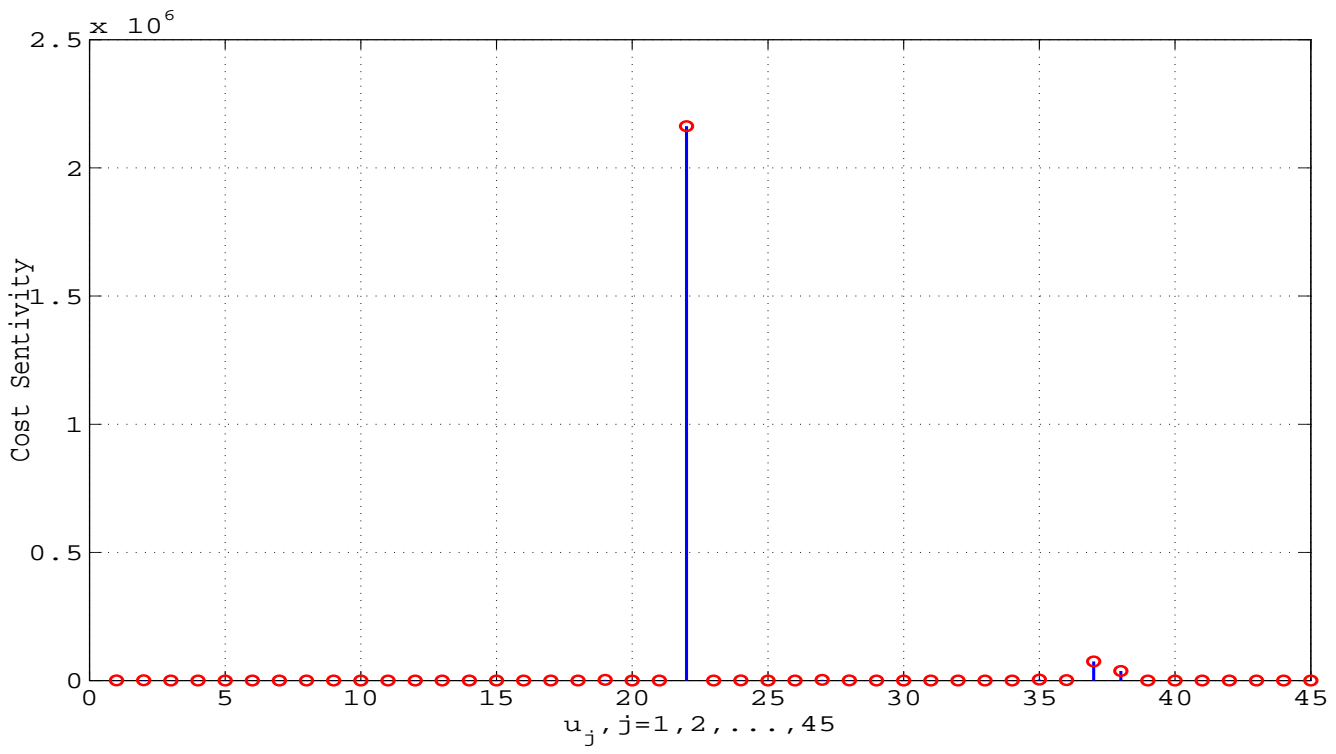

Fig. 15. The cost sensitivity for system parameters. 


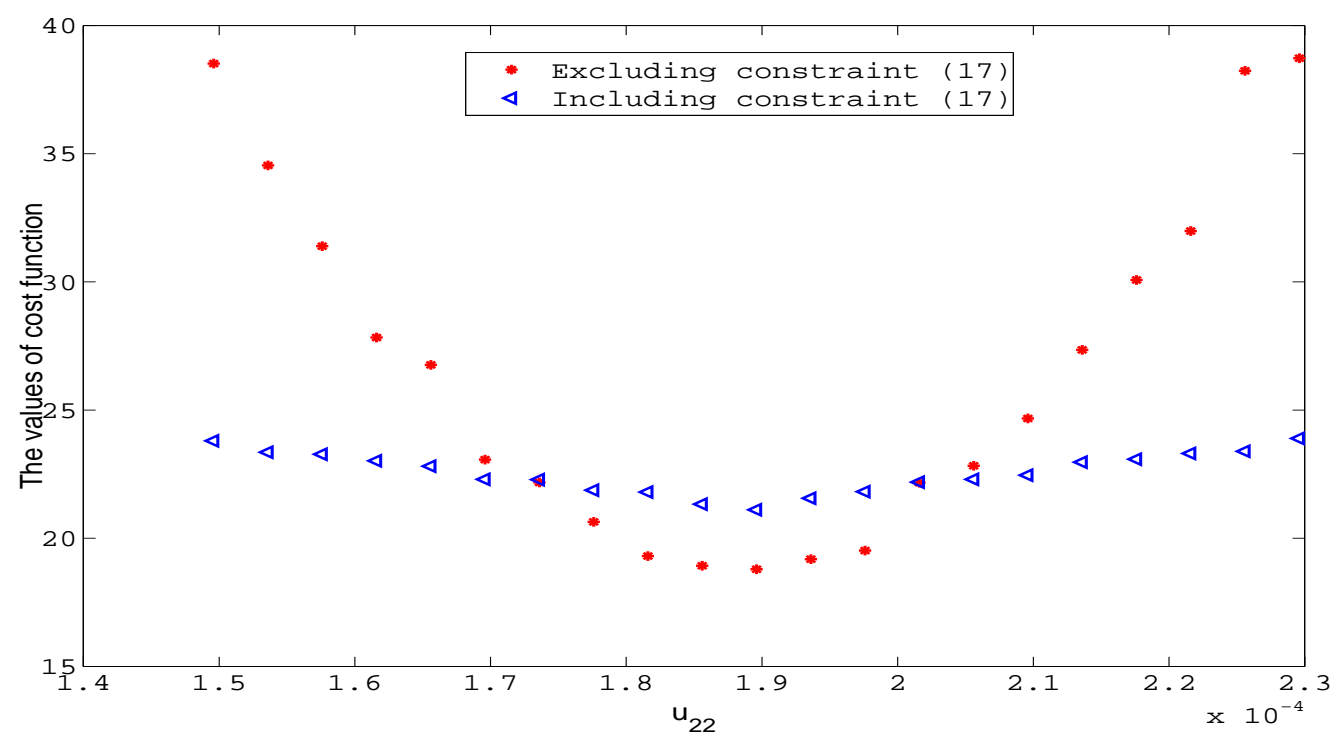

Fig. 16. Variation in the values of cost function due to $u_{22}$.

11401073 and 11501574), the Provincial Natural Science Foundation of Fujian (Grant Nos. 2014J05001), the Fundamental Research Funds for Central Universities in China (Grant Nos. DUT15LK25), the China Scholorship Council (CSC, No. 201506060121), the Social Science Fund of Liaoning Province of China (Grant Nos. L13BJY016) and the Humanity and Social Science Youth foundation of Ministry of Education of China (Grant Nos. 15YJC880002).

\section{Appendix A. Proof of Theorem 4}

Given $u \in \mathbb{R}^{45}$, for $\tau \in \mathcal{T}_{J}$, we have

$$
\frac{\mathrm{d} g_{i}(x(t \mid \tau, u))}{\mathrm{d} t}=\sum_{j=1}^{14} \frac{\partial g_{i}(x(t \mid \tau, u))}{\partial x_{j}} f_{j}\left(x, x_{\tau}, u\right), i \in I_{28} .
$$

It follows from (32) that $g_{i}(x(t \mid \tau, u)), i \in I_{28}$, is continuously differentiable. Then, there exists a positive constant $m_{i}, i \in I_{28}$, such that, for all $\tau \in \mathcal{T}_{J}$,

$$
\left|\frac{\mathrm{d} g_{i}(x(t \mid \tau, u))}{\mathrm{d} t}\right| \leq m_{i}, \forall t \in[0, T], i \in I_{28}
$$

Furthermore, for $\varepsilon>0$, define

$$
k_{\varepsilon, i}:=\frac{\varepsilon}{16} \min \left\{T, \frac{\varepsilon}{2 m_{i}}\right\}, i \in I_{28} .
$$

It suffices to show that $\mathcal{T}_{J}^{\varepsilon, \gamma, i} \subset \mathcal{T}_{J}^{i}$, for any such that

$$
0<\gamma<k_{\varepsilon, i}, i \in I_{28}
$$

where

and

$$
\mathcal{T}_{J}^{\varepsilon, \gamma, i}:=\left\{\tau \in \mathcal{T}_{J} \mid \tilde{G}_{\varepsilon, \gamma}^{i}(\tau \mid u) \geq 0, \operatorname{SRE}(\tau \mid u) \leq \sigma_{1},\left[\frac{\partial J(\tau \mid u)}{\partial u}\right]\left[\frac{\partial J(\tau \mid u)}{\partial u}\right]^{\mathrm{T}} \leq \sigma_{2}, i \in I_{28},\right\}
$$

$$
\mathcal{T}_{J}^{i}:=\left\{\tau \in \mathcal{T}_{J} \mid G^{i}(\tau \mid u)=0, \operatorname{SRE}(\tau \mid u) \leq \sigma_{1},\left[\frac{\partial J(\tau \mid u)}{\partial u}\right]\left[\frac{\partial J(\tau \mid u)}{\partial u}\right]^{\mathrm{T}} \leq \sigma_{2}\right\}, i \in I_{28}
$$


Assume the contrary. Then there exists an $\tau \in \mathcal{T}_{J}$ such that

$$
\begin{aligned}
\operatorname{SRE}(\tau \mid u) & \leq \sigma_{1} \\
{\left[\frac{\partial J(\tau \mid u)}{\partial u}\right]\left[\frac{\partial J(\tau \mid u)}{\partial u}\right]^{\mathrm{T}} } & \leq \sigma_{2} \\
\int_{0}^{T} \varphi_{\varepsilon, i}\left(g_{i}(x(t \mid \tau, u))\right) \mathrm{d} t+\gamma & \geq 0, \quad i \in I_{28}
\end{aligned}
$$

but

$$
\begin{aligned}
\operatorname{SRE}(\tau \mid u) & \leq \sigma_{1} \\
{\left[\frac{\partial J(\tau \mid u)}{\partial u}\right]\left[\frac{\partial J(\tau \mid u)}{\partial u}\right]^{\mathrm{T}} } & \leq \sigma_{2} \\
\int_{0}^{T} \min \left\{0, g_{i}(x(t \mid \tau, u))\right\} \mathrm{d} t & <0, \quad i \in I_{28} .
\end{aligned}
$$

Since $g_{i}(x(t \mid \tau, u)), i \in I_{28}$, is a continuous function of $t$ in $[0, T],(43)$ implies that there exists a $\bar{t} \in[0, T]$ such that

$$
g_{i}(x(\bar{t} \mid \tau, u))<0, i \in I_{28}
$$

Again by continuity, for each $i \in I_{28}$, there exists an interval $I_{i} \subset[0, T]$ containing $\bar{t}$ such that

$$
g_{i}(x(t \mid \tau, u))<\frac{\varepsilon}{2}, \forall t \in I_{i} .
$$

Using (39) it is clear from (45) that the length $I_{i}$ of the interval $I_{i}$ must satisfy

$$
\left|I_{i}\right| \geq \min \left\{T, \frac{\varepsilon}{2 m_{i}}\right\}, i \in I_{28}
$$

From the fact that $\varphi_{\varepsilon, i}\left(g_{i}\left(x\left(t \mid \tau_{\varepsilon, \gamma}, u\right)\right)\right), i \in I_{28}$, is non-positive, it follows from (42)

$$
0 \leq \gamma+\int_{0}^{T} \varphi_{\varepsilon, i}\left(g_{i}(x(t \mid \tau, u))\right) \mathrm{d} t \leq \gamma+\int_{I_{i}} \varphi_{\varepsilon, i}\left(g_{i}(x(t \mid \tau, u))\right) \mathrm{d} t \leq \gamma+\max _{t \in I_{i}}\left\{\varphi_{\varepsilon, i}\left(g_{i}(x(t \mid \tau, u))\right)\right\}\left|I_{i}\right|, i \in I_{28} .
$$

Now, in view of (45) and the monotony of the function $\varphi_{\varepsilon, i}\left(g_{i}\left(x\left(t \mid \tau_{\varepsilon, \gamma}, u\right)\right)\right), i \in I_{28}$, we have

$$
\max _{t \in I_{i}}\left\{\varphi_{\varepsilon, i}\left(g_{i}(x(t \mid \tau, u))\right)\right\}<-\frac{\varepsilon}{16}, i \in I_{28}
$$

Combining (40), (41), (46), (47) and (48), we have

$$
0 \leq \gamma+\int_{0}^{T} \varphi_{\varepsilon, i}\left(g_{i}(x(t \mid \tau, u))\right) \mathrm{d} t<\gamma-\frac{\varepsilon}{16} \min \left\{T, \frac{\varepsilon}{2 m_{i}}\right\}=\gamma-k_{\varepsilon, i}<0, i \in I_{28} .
$$

This is a contradiction. Thus, this completes the proof.

\section{Appendix B. Proof of Theorem 5}

Let $\tau \in \mathcal{T}_{J}$ be an arbitrary but fixed vector and $\bar{\delta}_{j}, j=3,6,7,8$, an arbitrary real number. Define

$$
\begin{aligned}
\tau^{3}(\bar{\rho}) & :=\left(\tau_{3}+\bar{\rho} \bar{\delta}_{3}, \tau_{6}, \tau_{7}, \tau_{8}\right), \\
\tau^{6}(\bar{\rho}) & :=\left(\tau_{3}, \tau_{6}+\bar{\rho} \bar{\delta}_{6}, \tau_{7}, \tau_{8}\right), \\
\tau^{7}(\bar{\rho}) & :=\left(\tau_{3}, \tau_{6}, \tau_{7}+\bar{\rho} \bar{\delta}_{3}, \tau_{8}\right), \\
\tau^{8}(\bar{\rho}) & :=\left(\tau_{3}, \tau_{6}, \tau_{7}, \tau_{8}+\bar{\rho} \bar{\delta}_{8}\right),
\end{aligned}
$$


For brevity, let $x(t \mid \tau, u)$ and $x\left(t \mid \tau^{j}(\bar{\rho}), u\right), j=3,6,7,8$, respectively denote the solutions of system (1) with $u \in \mathbb{R}^{45}$, $t \in(0, T]$. Clearly, we get

$$
\begin{aligned}
& x(t \mid \tau, u):=\quad x_{0}+\int_{0}^{t} f\left(x(s \mid \tau, u), x\left(s-\tau_{3} \mid \tau, u\right), x\left(s-\tau_{6} \mid \tau, u\right), x\left(s-\tau_{7} \mid \tau, u\right), x\left(s-\tau_{8} \mid \tau, u\right), u\right) \mathrm{d} s, \\
& x\left(t \mid \tau^{3}(\bar{\rho}), u\right):=x_{0}+\int_{0}^{t} f\left(x(s \mid \tau, u), x\left(s-\tau_{3}-\bar{\rho} \bar{\delta}_{3} \mid \tau, u\right), x\left(s-\tau_{6} \mid \tau, u\right), x\left(s-\tau_{7} \mid \tau, u\right), x\left(s-\tau_{8} \mid \tau, u\right), u\right) \mathrm{d} s, \\
& x\left(t \mid \tau^{6}(\bar{\rho}), u\right):=x_{0}+\int_{0}^{t} f\left(x(s \mid \tau, u), x\left(s-\tau_{3} \mid \tau, u\right), x\left(s-\tau_{6}-\bar{\rho} \bar{\delta}_{6} \mid \tau, u\right), x\left(s-\tau_{7} \mid \tau, u\right), x\left(s-\tau_{8} \mid \tau, u\right), u\right) \mathrm{d} s, \\
& x\left(t \mid \tau^{7}(\bar{\rho}), u\right):=x_{0}+\int_{0}^{t} f\left(x(s \mid \tau, u), x\left(s-\tau_{3} \mid \tau, u\right), x\left(s-\tau_{6} \mid \tau, u\right), x\left(s-\tau_{7}-\bar{\rho} \bar{\delta}_{7} \mid \tau, u\right), x\left(s-\tau_{8} \mid \tau, u\right), u\right) \mathrm{d} s, \\
& x\left(t \mid \tau^{8}(\bar{\rho}), u\right):=\quad x_{0}+\int_{0}^{t} f\left(x(s \mid \tau, u), x\left(s-\tau_{3} \mid \tau, u\right), x\left(s-\tau_{6} \mid \tau, u\right), x\left(s-\tau_{7} \mid \tau, u\right), x\left(s-\tau_{8}-\bar{\rho} \bar{\delta}_{8} \mid \tau, u\right), u\right) \mathrm{d} s,
\end{aligned}
$$

Consequently, for $\forall t \in(0, T]$,

$$
\begin{aligned}
& \left.\frac{d x\left(t \mid \tau^{j}(\bar{\rho}), u\right)}{d \bar{\rho}}\right|_{\bar{\rho}=0}=\frac{\partial x(t \mid \tau, u)}{\partial \tau_{j}}=\int_{0}^{t}\left\{\frac{\partial f\left(x(s \mid \tau, u), x_{\tau}, u\right)}{\partial x(s \mid \tau, u)} \frac{\partial x(s \mid \tau, u)}{\partial \tau_{j}}+\right. \\
& \left.\frac{\partial f\left(x(s \mid \tau, u), x_{\tau}, u\right)}{\partial x\left(s-\tau_{j} \mid \tau, u\right)} \frac{\partial x\left(s-\tau_{j} \mid \tau, u\right)}{\partial \tau_{j}}-\frac{\partial f\left(x(s \mid \tau, u), x_{\tau}, u\right)}{\partial x\left(s-\tau_{j} \mid \tau, u\right)} \chi\left(s-\tau_{j}\right)\right\} \mathrm{d} s, j=3,6,7,8 .
\end{aligned}
$$

For $\forall t \in(0, T]$, differentiating (49) with respect to time yields

$$
\begin{aligned}
\frac{\mathrm{d}}{\mathrm{d} t}\left\{\frac{\partial x(t \mid \tau, u)}{\partial \tau_{j}}\right\}= & \frac{\partial f\left(x(t \mid \tau, u), x_{\tau}, u\right)}{\partial x(t \mid \tau, u)} \frac{\partial x(t \mid \tau, u)}{\partial \tau_{j}}+ \\
& \frac{\partial f\left(x(t \mid \tau, u), x_{\tau}, u\right)}{\partial x\left(t-\tau_{j} \mid \tau, u\right)} \frac{\partial x\left(t-\tau_{j} \mid \tau, u\right)}{\partial \tau_{j}}-\frac{\partial f\left(x(t \mid \tau, u), x_{\tau}, u\right)}{\partial x\left(t-\tau_{j} \mid \tau, u\right)} \chi\left(t-\tau_{j}\right), j=3,6,7,8 .
\end{aligned}
$$

Moreover,

$$
\frac{\partial x(t \mid \tau, u)}{\partial \tau_{j}}=\frac{\partial}{\partial \tau_{j}}\{\phi(t)\}=\mathbf{0} \in \mathbb{R}^{14}, \quad \forall t \in[-\bar{\tau}, 0], j=3,6,7,8,
$$

Therefore, given $u \in \mathbb{R}^{45}$, for each $t \in(0, T]$, define

$$
\frac{\partial x(t \mid \tau, u)}{\partial \tau_{j}}=\varphi_{j}(t \mid \tau, u), \tau \in \mathcal{T}_{J}, j=3,6,7,8,
$$

and differentiate $\tilde{G}_{\varepsilon, \gamma}^{i}(\tau \mid u)$ with respect to $\tau$, we obtain the conclusion (35). Thus, this completes the proof. 


\section{Appendix C. The explicit formulas for some derivatives}

The explicit formulas for the derivatives of $\mu, q_{2}, q_{3}, q_{4}, q_{5}, U_{G}$ and $U_{P}$ with respect to $x_{1}, \ldots, x_{14}$ are given below. For $l_{1}=2,4,5, l_{4}=3,4,5, l_{2}=6, \ldots, 14, l_{3}=6,8, \ldots, 14, l_{5}=4,5$,

$$
\begin{aligned}
& \frac{\partial \mu}{\partial x_{1}}=0, \frac{\partial \mu}{\partial x_{2}}=\frac{u_{1}}{x_{2}+u_{2}} \prod_{j=3,7}\left(1-\frac{x_{j}\left(t-\tau_{j}\right)}{x_{j}^{*}}\right) \prod_{j=4}^{5}\left(1-\frac{x_{j}}{x_{j}^{*}}\right)\left[\left(1-\frac{x_{2}}{x_{2}^{*}}\right) \frac{u_{2}}{x_{2}+u_{2}}-x_{2}\right], \frac{\partial \mu}{\partial x_{3}}=0, \\
& \frac{\partial \mu}{\partial x_{3}\left(t-\tau_{3}\right)}=-\frac{u_{1} x_{2}}{x_{2}+u_{2}}\left(1-\frac{x_{7}\left(t-\tau_{7}\right)}{x_{7}^{*}}\right) \prod_{j=2, j \neq 3}^{5}\left(1-\frac{x_{j}}{x_{j}^{*}}\right), \frac{\partial \mu}{\partial x_{4}}=-\frac{u_{1} x_{2}}{x_{2}+u_{2}} \prod_{j=3,7}\left(1-\frac{x_{j}\left(t-\tau_{j}\right)}{x_{j}^{*}}\right) \prod_{j=2, j \neq 4}^{5}\left(1-\frac{x_{j}}{x_{j}^{*}}\right), \\
& \frac{\partial \mu}{\partial x_{5}}=-\frac{u_{1} x_{2}}{x_{2}+u_{2}} \prod_{j=3,7}\left(1-\frac{x_{j}\left(t-\tau_{j}\right)}{x_{j}^{*}}\right) \prod_{j=2, j \neq 5}^{5}\left(1-\frac{x_{j}}{x_{j}^{*}}\right), \frac{\partial q_{2}}{\partial x_{3}\left(t-\tau_{3}\right)}=\frac{\partial \mu}{\partial x_{3}\left(t-\tau_{3}\right)} \frac{1}{u_{4}}, \frac{\partial q_{l_{4}}}{\partial x_{3}\left(t-\tau_{3}\right)}=\frac{\partial \mu}{\partial x_{3}\left(t-\tau_{3}\right)} u_{2 l_{4}}, \\
& \frac{\partial q_{2}}{\partial x_{l_{1}}}=\frac{\partial \mu}{\partial x_{l_{1}}} \frac{1}{u_{4}}, \frac{\partial q_{l_{4}}}{\partial x_{l_{1}}}=\frac{\partial \mu}{\partial x_{l_{1}}} u_{2 l_{4}}, \frac{\partial U_{G}}{\partial x_{3}\left(t-\tau_{3}\right)}=-u_{12} \frac{\partial \mu}{\partial x_{3}\left(t-\tau_{3}\right)}, \frac{\partial U_{G}}{\partial x_{k}}=-u_{12} \frac{\partial \mu}{\partial x_{k}}, \frac{\partial U_{G}}{\partial x_{2}}=-u_{12} \frac{\partial \mu}{\partial x_{2}}-\frac{u_{13}}{x_{2}+u_{14}}+\frac{u_{13} x_{2}}{\left(x_{2}+u_{14}\right)^{2}} \\
& \frac{\partial U_{P}}{\partial x_{3}\left(t-\tau_{3}\right)}=-u_{16} \frac{\partial \mu}{\partial x_{3}\left(t-\tau_{3}\right)}, \frac{\partial U_{P}}{\partial x_{k}}=-u_{16} \frac{\partial \mu}{\partial x_{k}}, \frac{\partial U_{P}}{\partial x_{2}}=-u_{16} \frac{\partial \mu}{\partial x_{2}}-\frac{u_{17}}{x_{2}+u_{18}}+\frac{u_{17} x_{2}}{\left(x_{2}+u_{18}\right)^{2}}, \frac{\partial \mu}{\partial x_{l_{3}}}=\frac{\partial q_{l_{1}}}{\partial x_{l_{2}}}=0 .
\end{aligned}
$$

The explicit formulas for the derivatives of $\mu, q_{2}, q_{3}, q_{4}, q_{5}, U_{G}$ and $U_{P}$ with respect to $u_{1}, \ldots, u_{45}$ are given below. For $\ell_{1}=3, \ldots, 45, \ell_{2}=5, \ldots, 45, \ell_{3}=3, \ldots, 5, \ell_{4}=3,4,7, \ldots, 45, \ell_{5}=3, \ldots, 6,9, \ldots, 45, \ell_{6}=3, \ldots, 8,11, \ldots, 45, \ell_{7}=$ $3, \ldots, 10,15, \ldots, 45, \ell_{8}=3, \ldots, 14,19, \ldots, 45$,

$$
\begin{aligned}
& \frac{\partial \mu}{\partial u_{1}}=\frac{x_{2}}{x_{2}+u_{2}} \prod_{j=3,7}\left(1-\frac{x_{j}\left(t-\tau_{j}\right)}{x_{j}^{*}}\right) \prod_{j=2, j \neq 3}^{5}\left(1-\frac{x_{j}}{x_{j}^{*}}\right), \frac{\partial \mu}{\partial u_{2}}=-\frac{u_{1} x_{2}}{\left(x_{2}+u_{2}\right)^{2}} \prod_{j=3,7}\left(1-\frac{x_{j}\left(t-\tau_{j}\right)}{x_{j}^{*}}\right) \prod_{j=2, j \neq 3}^{5}\left(1-\frac{x_{j}}{x_{j}^{*}}\right), \\
& \frac{\partial \mu}{\partial u_{\ell_{1}}}=0, \frac{\partial q_{2}}{\partial u_{1}}=\frac{1}{u_{4}} \frac{\partial \mu}{\partial u_{1}}, \frac{\partial q_{2}}{\partial u_{2}}=\frac{1}{u_{4}} \frac{\partial \mu}{\partial u_{2}}, \\
& \frac{\partial q_{2}}{\partial u_{3}}=1, \frac{\partial q_{2}}{\partial u_{4}}=-\frac{\mu}{u_{4}^{2}}, \frac{\partial q_{2}}{\partial u_{\ell_{2}}}=0, \frac{\partial q_{\ell_{3}}}{\partial u_{1}}=u_{2 \ell_{3}} \frac{\partial \mu}{\partial u_{1}}, \frac{\partial q_{\ell_{3}}}{\partial u_{2}}=u_{2 \ell_{3}} \frac{\partial \mu}{\partial u_{2}}, \frac{\partial q_{3}}{\partial u_{5}}=\frac{\partial q_{4}}{\partial u_{7}}=\frac{\partial q_{5}}{\partial u_{9}}=1, \frac{\partial q_{\ell_{3}}}{\partial u_{2 \ell_{3}}}=\mu, \\
& \frac{\partial q_{3}}{\partial u_{\ell_{4}}}=\frac{\partial q_{4}}{\partial u_{\ell_{5}}}=\frac{\partial q_{5}}{\partial u_{\ell_{6}}}=0, \frac{\partial U_{G}}{\partial u_{1}}=-u_{12} \frac{\partial \mu}{\partial u_{1}}, \frac{\partial U_{G}}{\partial u_{2}}=-u_{12} \frac{\partial \mu}{\partial u_{2}}, \frac{\partial U_{G}}{\partial u_{11}}=1, \frac{\partial U_{G}}{\partial u_{12}}=-\mu, \frac{\partial U_{G}}{\partial u_{13}}=-\frac{x_{2}}{x_{2}+u_{14}}, \\
& \frac{\partial U_{G}}{\partial u_{14}}=\frac{u_{13} x_{2}}{\left(x_{2}+u_{14}\right)^{2}}, \frac{\partial U_{P}}{\partial u_{1}}=-u_{16} \frac{\partial \mu}{\partial u_{1}}, \frac{\partial U_{P}}{\partial u_{2}}=-u_{16} \frac{\partial \mu}{\partial u_{2}}, \frac{\partial U_{P}}{\partial u_{15}}=1, \frac{\partial U_{P}}{\partial u_{16}}=-\mu, \frac{\partial U_{P}}{\partial u_{17}}=-\frac{x_{2}}{x_{2}+u_{18}}, \\
& \frac{\partial U_{P}}{\partial u_{18}}=\frac{u_{17} x_{2}}{\left(x_{2}+u_{18}\right)^{2}}, \frac{\partial U_{G}}{\partial u_{\ell_{7}}}=\frac{\partial U_{P}}{\partial u_{\ell_{8}}}=0 .
\end{aligned}
$$

The explicit formulas for $\frac{\partial f\left(x(t \mid \tau, u), x_{\tau}, u\right)}{\partial u} \in \mathbb{R}^{14 \times 45}$ in (30), denoted by $\frac{\partial f}{\partial u}$, are given below. For $\ell_{9}=$ $1,2, \ell_{10}=1, \ldots, 4, \ell_{11}=1,2,5,6, \ell_{12}=1,2,7,8, \ell_{13}=1,2,9,10, \ell_{14}=1,2,11, \ldots, 14, \ell_{15}=1,2,15, \ldots, 18, \ell_{16}=$ $5, \ldots, 18,23, \ldots, 45, \ell_{17}=11, \ldots, 14, \ell_{18}=15, \ldots, 18, \ell_{19}=3, \ldots, 10,19, \ldots, 22,31, \ldots, 45, \ell_{20}=3, \ldots, 29,31, \ldots, 33$, $36, \ldots, 45, \ell_{21}=3, \ldots, 29,31, \ldots, 35,40, \ldots, 45, \ell_{22}=3, \ldots, 29,31, \ldots, 33,35, \ldots, 38,40, \ldots, 45, \ell_{23}=3, \ldots, 40,43,45$, 
$\ell_{24}=3, \ldots, 29,31, \ldots, 33,35, \ldots, 42,44,45, \ell_{25}=3, \ldots, 43, \ell_{26}=3, \ldots, 14,19, \ldots, 22,24,25,29,30,34, \ldots, 45$, $\frac{\partial f_{1}}{\partial u_{\ell_{9}}}=x_{1} \frac{\partial \mu}{\partial u_{\ell_{9}}}, \frac{\partial f_{1}}{\partial u_{\ell_{1}}}=0 ; \frac{\partial f_{2}}{\partial u_{\ell_{10}}}=-x_{1} \frac{\partial q_{2}}{\partial u_{\ell_{10}}}, \frac{\partial f_{2}}{\partial u_{\ell_{2}}}=0 ; \frac{\partial f_{3}}{\partial u_{\ell_{11}}}=x_{1} \frac{\partial q_{3}}{\partial u_{\ell_{11}}}, \frac{\partial f_{3}}{\partial u_{\ell_{4}}}=0 ; \frac{\partial f_{4}}{\partial u_{\ell_{12}}}=x_{1} \frac{\partial q_{4}}{\partial u_{\ell_{12}}}, \frac{\partial f_{4}}{\partial u_{\ell_{5}}}=0 ;$ $\frac{\partial f_{5}}{\partial u_{\ell_{13}}}=x_{1} \frac{\partial q_{5}}{\partial u_{\ell_{13}}}, \frac{\partial f_{5}}{\partial u_{\ell_{6}}}=0 ; \frac{\partial f_{6}}{\partial u_{\ell_{9}}}=\frac{-1}{u_{19}} \frac{\partial q_{2}}{\partial u_{\ell_{9}}}-x_{6}\left(t-\tau_{6}\right) \frac{\partial \mu}{\partial u_{\ell_{9}}}, \frac{\partial f_{6}}{\partial u_{3}}=\frac{-1}{u_{19}} \frac{\partial q_{2}}{\partial u_{3}}, \frac{\partial f_{6}}{\partial u_{4}}=\frac{-1}{u_{19}} \frac{\partial q_{2}}{\partial u_{4}}$, $\frac{\partial f_{6}}{\partial u_{19}}=\frac{-1}{u_{19}^{2}}\left[u_{20} \frac{x_{2}}{x_{2}+u_{21}}+\frac{1}{u_{22}}\left(x_{2}-x_{6}\left(t-\tau_{6}\right)\right)-q_{2}\right], \frac{\partial f_{6}}{\partial u_{20}}=\frac{x_{2}}{u_{19}\left(x_{2}+u_{21}\right)}, \frac{\partial f_{6}}{\partial u_{21}}=\frac{-u_{20} x_{2}}{u_{19}\left(x_{2}+u_{21}\right)^{2}}$, $\frac{\partial f_{6}}{\partial u_{22}}=-\frac{x_{2}-x_{6}\left(t-\tau_{6}\right)}{u_{19} u_{22}^{2}} \frac{\partial f_{6}}{\partial u_{\ell_{16}}}=0$

$\frac{\partial f_{7}}{\partial u_{\ell_{9}}}=-x_{6}\left(t-\tau_{6}\right) \frac{\partial \mu}{\partial u_{\ell_{9}}}+\frac{\partial U_{G}}{\partial u_{\ell_{9}}} \frac{u_{23} \times x_{6}\left(t-\tau_{6}\right) x_{12}}{u_{24}\left(1+\frac{x_{7}\left(t-\tau_{7}\right)}{u_{25}}\right)+x_{6}\left(t-\tau_{6}\right)}-\frac{\partial U_{P}}{\partial u_{\ell_{9}}} \frac{u_{26} \times x_{7}\left(t-\tau_{7}\right) x_{14}}{u_{27}+x_{7}\left(t-\tau_{7}\right)\left(1+\frac{x_{7}\left(t-\tau_{7}\right)}{u_{28}}\right)}$, $\frac{\partial f_{7}}{\partial u_{\ell_{17}}}=\frac{\partial U_{G}}{\partial u_{\ell_{17}}} \frac{u_{23} \times x_{6}\left(t-\tau_{6}\right) x_{12}}{u_{24}\left(1+\frac{x_{7}\left(t-\tau_{7}\right)}{u_{25}}\right)+x_{6}\left(t-\tau_{6}\right)}, \frac{\partial f_{7}}{\partial u_{\ell_{18}}}=-\frac{\partial U_{P}}{\partial u_{\ell_{18}}} \frac{u_{26} \times x_{7}\left(t-\tau_{7}\right) x_{14}}{u_{27}+x_{7}\left(t-\tau_{7}\right)\left(1+\frac{x_{7}\left(t-\tau_{7}\right)}{u_{28}}\right)}$,

$\frac{\partial f_{7}}{\partial u_{23}}=\frac{U_{G} x_{6}\left(t-\tau_{6}\right) x_{12}}{u_{24}\left(1+\frac{x_{7}\left(t-\tau_{7}\right)}{u_{25}}\right)+x_{6}\left(t-\tau_{6}\right)}, \frac{\partial f_{7}}{\partial u_{24}}=-\frac{u_{23} U_{G} x_{6}\left(t-\tau_{6}\right) x_{12}\left(1+\frac{x_{7}\left(t-\tau_{7}\right)}{u_{25}}\right)}{\left[u_{24}\left(1+\frac{x_{7}\left(t-\tau_{7}\right)}{u_{25}}\right)+x_{6}\left(t-\tau_{6}\right)\right]^{2}}$, $\frac{\partial f_{7}}{\partial u_{25}}=\frac{u_{23} U_{G} x_{6}\left(t-\tau_{6}\right) x_{12}\left(\frac{u_{24} x_{7}\left(t-\tau_{7}\right)}{u_{25}^{2}}\right)}{\left[u_{24}\left(1+\frac{x_{7}\left(t-\tau_{7}\right)}{u_{25}}\right)+x_{6}\left(t-\tau_{6}\right)\right]^{2}}, \frac{\partial f_{7}}{\partial u_{26}}=-\frac{U_{P} x_{7}\left(t-\tau_{7}\right) x_{14}}{u_{27}+x_{7}\left(t-\tau_{7}\right)\left(1+\frac{x_{7}\left(t-\tau_{7}\right)}{u_{28}}\right)}$,

$\frac{\partial f_{7}}{\partial u_{27}}=\frac{u_{26} U_{P} x_{7}\left(t-\tau_{7}\right) x_{14}}{\left[u_{27}+x_{7}\left(t-\tau_{7}\right)\left(1+\frac{x_{7}\left(t-\tau_{7}\right)}{u_{28}}\right)\right]^{2}}, \frac{\partial f_{7}}{\partial u_{28}}=-\frac{u_{26} U_{P} x_{7}\left(t-\tau_{7}\right) x_{14}}{\left[u_{27}+x_{7}\left(t-\tau_{7}\right)\left(1+\frac{x_{7}\left(t-\tau_{7}\right)}{u_{28}}\right)\right]^{2}} \frac{x_{7}\left(t-\tau_{7}\right)^{2}}{u_{28}^{2}}$, $\frac{\partial f_{7}}{\partial u_{29}}=-\frac{x_{7}\left(t-\tau_{7}\right)}{x_{7}\left(t-\tau_{7}\right)+u_{30}}, \frac{\partial f_{7}}{\partial u_{30}}=\frac{u_{29} x_{7}\left(t-\tau_{7}\right)}{\left(x_{7}\left(t-\tau_{7}\right)+u_{30}\right)^{2}}, \frac{\partial f_{7}}{\partial u_{\ell_{19}}}=0$;

$\frac{\partial f_{8}}{\partial u_{\ell_{9}}}=\frac{\partial U_{P}}{\partial u_{\ell_{9}}} \frac{u_{26} \times x_{7}\left(t-\tau_{7}\right) x_{14}}{u_{27}+x_{7}\left(t-\tau_{7}\right)\left(1+\frac{x_{7}\left(t-\tau_{7}\right)}{u_{28}}\right)}-x_{8}\left(t-\tau_{8}\right) \frac{\partial \mu}{\partial u_{\ell_{9}}}, \frac{\partial f_{8}}{\partial u_{\ell_{18}}}=\frac{\partial U_{P}}{\partial u_{\ell_{18}}} \frac{u_{26} \times x_{7}\left(t-\tau_{7}\right) x_{14}}{u_{27}+x_{7}\left(t-\tau_{7}\right)\left(1+\frac{x_{7}\left(t-\tau_{7}\right)}{u_{28}}\right)}$,

$\frac{\partial f_{8}}{\partial u_{23}}=\frac{u_{26} U_{P} x_{7}\left(t-\tau_{7}\right) x_{14}}{u_{27}+x_{7}\left(t-\tau_{7}\right)\left(1+\frac{x_{7}\left(t-\tau_{7}\right)}{u_{28}}\right)}, \frac{\partial f_{8}}{\partial u_{26}}=\frac{u_{23} U_{P} x_{7}\left(t-\tau_{7}\right) x_{14}}{u_{27}+x_{7}\left(t-\tau_{7}\right)\left(1+\frac{x_{7}\left(t-\tau_{7}\right)}{u_{28}}\right)}, \frac{\partial f_{8}}{\partial u_{27}}=-\frac{u_{23} U_{P} x_{7}\left(t-\tau_{7}\right) x_{14}}{\left[u_{27}+x_{7}\left(t-\tau_{7}\right)\left(1+\frac{x_{7}\left(t-\tau_{7}\right)}{u_{28}}\right)\right]^{2}}$, $\frac{\partial f_{8}}{\partial u_{28}}=\frac{u_{23} U_{P} x_{7}\left(t-\tau_{7}\right) x_{14}\left(\frac{x_{7}\left(t-\tau_{7}\right)^{2}}{u_{28}^{2}}\right)}{\left[u_{27}+x_{7}\left(t-\tau_{7}\right)\left(1+\frac{x_{7}\left(t-\tau_{7}\right)}{u_{28}}\right)\right]^{2}}, \frac{\partial f_{8}}{\partial u_{31}}=-\frac{x_{8}\left(t-\tau_{8}\right)}{x_{8}\left(t-\tau_{8}\right)+u_{32}}, \frac{\partial f_{8}}{\partial u_{32}}=\frac{u_{31} x_{8}\left(t-\tau_{8}\right)}{\left(x_{8}\left(t-\tau_{8}\right)+u_{32}\right)^{2}}$, $\frac{\partial f_{8}}{\partial u_{33}}=-\left(x_{8}\left(t-\tau_{8}\right)-x_{3}\right), \frac{\partial f_{8}}{\partial u_{\ell_{26}}}=0 ; \frac{\partial f_{9}}{\partial u_{\ell_{9}}}=-x_{9} \frac{\partial \mu}{\partial u_{\ell_{9}}}$,

$\frac{\partial f_{9}}{\partial u_{30}}=\frac{1}{x_{7}\left(t-\tau_{7}\right)+u_{30}+u_{34} x_{7}\left(t-\tau_{7}\right)}-\frac{x_{7}\left(t-\tau_{7}\right)+u_{30}}{\left(x_{7}\left(t-\tau_{7}\right)+u_{30}+u_{34} x_{7}\left(t-\tau_{7}\right)\right)^{2}}, \frac{\partial f_{9}}{\partial u_{34}}=-\frac{x_{7}\left(t-\tau_{7}\right)\left(x_{7}\left(t-\tau_{7}\right)+u_{30}\right)}{\left(x_{7}\left(t-\tau_{7}\right)+u_{30}+u_{34} x_{7}\left(t-\tau_{7}\right)\right)^{2}}$ $\frac{\partial f_{9}}{\partial u_{35}}=-x_{9}, \frac{\partial f_{9}}{\partial u_{\ell_{20}}}=0 ; \frac{\partial f_{10}}{\partial u_{\ell_{9}}}=-x_{10} \frac{\partial \mu}{\partial u_{\ell_{9}}}, \frac{\partial f_{10}}{\partial u_{36}}=x_{6}\left(t-\tau_{6}\right), \frac{\partial f_{10}}{\partial u_{37}}=-\frac{u_{38} x_{7}\left(t-\tau_{7}\right)}{x_{7}\left(t-\tau_{7}\right)+u_{30}}, \frac{\partial f_{10}}{\partial u_{38}}=-\frac{u_{37} x_{7}\left(t-\tau_{7}\right)}{x_{7}\left(t-\tau_{7}\right)+u_{30}}$, $\frac{\partial f_{10}}{\partial u_{30}}=\frac{u_{37} u_{38} x_{7}\left(t-\tau_{7}\right)}{\left(x_{7}\left(t-\tau_{7}\right)+u_{30}\right)^{2}}, \frac{\partial f_{10}}{\partial u_{39}}=-x_{10}, \frac{\partial f_{10}}{\partial u_{\ell_{21}}}=0 ; \frac{\partial f_{11}}{\partial u_{\ell_{9}}}=-x_{11} \frac{\partial \mu}{\partial u_{\ell_{9}}}, \frac{\partial f_{11}}{\partial u_{30}}=\frac{\partial f_{9}}{\partial u_{30}}, \frac{\partial f_{11}}{\partial u_{34}}=\frac{\partial f_{9}}{\partial u_{34}}, \frac{\partial f_{11}}{\partial u_{39}}=-x_{11}, \frac{\partial f_{11}}{\partial u_{\ell_{22}}}=0$; $\frac{\partial f_{12}}{\partial u_{\ell_{9}}}=-x_{12} \frac{\partial \mu}{\partial u_{\ell_{9}}}, \frac{\partial f_{12}}{\partial u_{41}}=x_{11}, \frac{\partial f_{12}}{\partial u_{42}}=-x_{12}, \frac{\partial f_{12}}{\partial u_{\ell_{23}}}=\hat{\jmath} 1, \frac{\partial f_{13}}{\partial u_{\ell_{9}}}=-x_{13} \frac{\partial \mu}{\partial u_{\ell_{9}}}, \frac{\partial f_{13}}{\partial u_{30}}=\frac{\partial f_{11}}{\partial u_{30}}, \frac{\partial f_{13}}{\partial u_{34}}=\frac{\partial f_{11}}{\partial u_{34}}$, $\frac{\partial f_{13}}{\partial u_{43}}=-x_{13}, \frac{\partial f_{13}}{\partial u_{\ell_{24}}}=0 ; \frac{\partial f_{14}}{\partial u_{\ell_{9}}}=-x_{14} \frac{\partial \mu}{\partial u_{\ell_{9}}}, \frac{\partial f_{14}}{\partial u_{44}}=x_{14}, \frac{\partial f_{14}}{\partial u_{45}}=-x_{14}, \frac{\partial f_{14}}{\partial u_{\ell_{25}}}=0$. 
The explicit formulas for $\frac{\partial f\left(x(t \mid \tau, u), x_{\tau}, u\right)}{\partial x(t \mid \tau, u)}$ in (30), denoted by $\frac{\partial f}{\partial x}$ are given below. For $l_{1}=2, \ldots, 5, l_{2}=$ $6, \ldots, 14, l_{3}=3, \ldots, 5, l_{4}=7, \ldots, 14, l_{5}=8, \ldots, 13, l_{6}=2,4,5, l_{7}=9, \ldots, 13, l_{8}=10, \ldots, 14, l_{9}=11, \ldots, 14, l_{10}=$ $8, \ldots, 10, l_{11}=12, \ldots, 14, l_{12}=6, \ldots, 10, l_{13}=8, \ldots, 12, l_{14}=6, \ldots, 13$,

$$
\begin{aligned}
& \frac{\partial f_{1}}{\partial x_{1}}=\mu, \frac{\partial f_{1}}{\partial x_{l_{6}}}=x_{1} \frac{\partial \mu}{\partial x_{l_{6}}}, \frac{\partial f_{1}}{\partial x_{l_{2}}}=\frac{\partial f_{1}}{\partial x_{3}}=0, \frac{\partial f_{2}}{\partial x_{1}}=-q_{2}, \frac{\partial f_{2}}{\partial x_{l_{1}}}=-\frac{x_{1}}{u_{4}} \frac{\partial \mu}{\partial x_{l_{1}}}, \frac{\partial f_{2}}{\partial x_{l_{2}}}=0, \frac{\partial f_{l_{3}}}{\partial x_{1}}=q_{l_{3}}, \frac{\partial f_{l_{3}}}{\partial x_{l_{6}}}=x_{1} \frac{\partial q_{l_{3}}}{\partial x_{l_{6}}}, \frac{\partial f_{l_{3}}}{\partial x_{l_{2}}}=0, \\
& \frac{\partial f_{l_{3}}}{\partial x_{3}}=0, \frac{\partial f_{6}}{\partial x_{1}}=0, \frac{\partial f_{6}}{\partial x_{2}}=\frac{1}{u_{19}}\left[\frac{u_{20}}{x_{2}+u_{21}}-\frac{u_{20} x_{2}}{x_{2}+u_{21}}+\frac{1}{u_{22}}-\frac{\partial q_{2}}{\partial x_{2}}\right]-x_{6}\left(t-\tau_{6}\right) \frac{\partial \mu}{\partial x_{2}}, \frac{\partial f_{6}}{\partial x_{4}}=-x_{6}\left(t-\tau_{6}\right) \frac{\partial \mu}{\partial x_{4}}, \\
& \frac{\partial f_{6}}{\partial x_{5}}=-x_{6}\left(t-\tau_{6}\right) \frac{\partial \mu}{\partial x_{5}}, \frac{\partial f_{6}}{\partial x_{3}}=\frac{\partial f_{6}}{\partial x_{l_{2}}}=0, \frac{\partial f_{7}}{\partial x_{1}}=\frac{\partial f_{7}}{\partial x_{3}}=\frac{\partial f_{7}}{\partial x_{l_{2}}}=0, \frac{\partial f_{7}}{\partial x_{14}}=-\frac{u_{26} U_{P} x_{7}\left(t-\tau_{7}\right)}{u_{27}+x_{7}\left(t-\tau_{7}\right)\left(1+\frac{x_{7}\left(t-\tau_{7}\right)}{u_{28}}\right)} \text {, } \\
& \frac{\partial f_{7}}{\partial x_{l_{6}}}=-x_{7}\left(t-\tau_{7}\right) \frac{\partial \mu}{\partial x_{l_{6}}}+\frac{\partial U_{G}}{\partial x_{l_{6}}} \frac{u_{23} x_{6}\left(t-\tau_{6}\right) x_{12}}{u_{24}\left(1+\frac{x_{7}}{u_{25}}\right)+x_{6}\left(t-\tau_{6}\right)}-\frac{\partial U_{P}}{\partial x_{l_{6}}} \frac{u_{26} x_{7}\left(t-\tau_{7}\right) x_{14}}{u_{27}+x_{7}\left(t-\tau_{7}\right)\left(1+\frac{x_{7}\left(t-\tau_{7}\right)}{u_{28}}\right)}, \\
& \frac{\partial f_{8}}{\partial x_{1}}=\frac{\partial f_{8}}{\partial x_{3}}=\frac{\partial f_{8}}{\partial x_{6}}=\frac{\partial f_{8}}{\partial x_{7}}=0, \frac{\partial f_{8}}{\partial x_{l_{6}}}=-x_{8}\left(t-\tau_{8}\right) \frac{\partial \mu}{\partial x_{l_{6}}}+\frac{\partial U_{P}}{\partial x_{l_{6}}} \frac{u_{23} u_{26} x_{7}\left(t-\tau_{7}\right) x_{14}}{u_{27}+x_{7}\left(t-\tau_{7}\right)\left(1+\frac{x_{7}\left(t-\tau_{7}\right)}{u_{28}}\right)}, \\
& \frac{\partial f_{8}}{\partial x_{l_{5}}}=0, \frac{\partial f_{8}}{\partial x_{14}}=\frac{u_{23} u_{26} U_{P} x_{7}}{u_{27}+x_{7}\left(t-\tau_{7}\right)\left(1+\frac{x_{7}\left(t-\tau_{7}\right)}{u_{28}}\right)}, \frac{\partial f_{9}}{\partial x_{1}}=\frac{\partial f_{9}}{\partial x_{3}}=\frac{\partial f_{9}}{\partial x_{6}}=\frac{\partial f_{9}}{\partial x_{7}}=\frac{\partial f_{9}}{\partial x_{8}}=0, \frac{\partial f_{9}}{\partial x_{l_{6}}}=-x_{9} \frac{\partial \mu}{\partial x_{l_{6}}} \\
& \frac{\partial f_{9}}{\partial x_{9}}=-\left(u_{35}+\mu\right), \frac{\partial f_{9}}{\partial x_{l_{8}}}=0, \frac{\partial f_{10}}{\partial x_{1}}=\frac{\partial f_{10}}{\partial x_{3}}=\frac{\partial f_{10}}{\partial x_{6}}=0 \text {, } \\
& \frac{\partial f_{10}}{\partial x_{l_{6}}}=-x_{10} \frac{\partial \mu}{\partial x_{l_{6}}}, \frac{\partial f_{10}}{\partial x_{7}}=\frac{\partial f_{10}}{\partial x_{8}}=0, \frac{\partial f_{10}}{\partial x_{9}}=u_{36}, \frac{\partial f_{10}}{\partial x_{10}}=-\left(u_{39}+\mu\right), \frac{\partial f_{10}}{\partial x_{l_{9}}}=0, \\
& \frac{\partial f_{11}}{\partial x_{1}}=\frac{\partial f_{11}}{\partial x_{3}}=\frac{\partial f_{11}}{\partial x_{6}}=\frac{\partial f_{11}}{\partial x_{7}}=\frac{\partial f_{11}}{\partial x_{l_{10}}}=\frac{\partial f_{11}}{\partial x_{l_{11}}}=0, \frac{\partial f_{11}}{\partial x_{l_{6}}}=-x_{11} \frac{\partial \mu}{\partial x_{l_{6}}}, \frac{\partial f_{11}}{\partial x_{11}}=-\left(u_{40}+\mu\right), \\
& \frac{\partial f_{12}}{\partial x_{1}}=\frac{\partial f_{12}}{\partial x_{3}}=\frac{\partial f_{12}}{\partial x_{12}}=\frac{\partial f_{12}}{\partial x_{13}}=\frac{\partial f_{12}}{\partial x_{14}}=0, \frac{\partial f_{12}}{\partial x_{l_{6}}}=-x_{12} \frac{\partial \mu}{\partial x_{l_{6}}}, \frac{\partial f_{12}}{\partial x_{11}}=u_{41}, \\
& \frac{\partial f_{12}}{\partial x_{12}}=-\left(u_{42}+\mu\right), \frac{\partial f_{13}}{\partial x_{1}}=\frac{\partial f_{13}}{\partial x_{3}}=\frac{\partial f_{13}}{\partial x_{6}}=\frac{\partial f_{13}}{\partial x_{7}}=\frac{\partial f_{13}}{\partial x_{13}}=\frac{\partial f_{13}}{\partial x_{14}}=0, \frac{\partial f_{13}}{\partial x_{16}}=-x_{13} \frac{\partial \mu}{\partial x_{16}}, \\
& \frac{\partial f_{13}}{\partial x_{13}}=-\left(u_{43}+\mu\right), \frac{\partial f_{14}}{\partial x_{1}}=\frac{\partial f_{14}}{\partial x_{l_{3}}}=\frac{\partial f_{14}}{\partial x_{l_{14}}}=0, \frac{\partial f_{14}}{\partial x_{l_{6}}}=-x_{14} \frac{\partial \mu}{\partial x_{l_{6}}}, \frac{\partial f_{14}}{\partial x_{14}}=u_{44}-u_{45}-\mu \text {. }
\end{aligned}
$$

The explicit formulas for $\frac{\partial f\left(x(t \mid \tau, u), x_{\tau}, u\right)}{\partial x\left(t-\tau_{3} \mid \tau, u\right)}$ in (30), denoted by $\frac{\partial f}{\partial x\left(t-\tau_{3}\right)}$ are given below. For $l_{1}=2, \ldots, 5, l_{2}=$ $6, \ldots, 14, l_{3}=3, \ldots, 5, l_{4}=7, \ldots, 14, l_{5}=8, \ldots, 13, l_{6}=2,4,5, l_{7}=9, \ldots, 13, l_{8}=10, \ldots, 14, l_{9}=11, \ldots, 14, l_{10}=$ 
$8, \ldots, 10, l_{11}=12, \ldots, 14, l_{12}=6, \ldots, 10, l_{13}=8, \ldots, 12, l_{14}=6, \ldots, 13, l_{15}=9, \ldots, 14$

$$
\begin{aligned}
& \frac{\partial f_{1}}{\partial x_{1}}=\mu, \frac{\partial f_{1}}{\partial x_{3}\left(t-\tau_{3}\right)}=x_{1} \frac{\partial \mu}{\partial x_{3}\left(t-\tau_{3}\right)}, \frac{\partial f_{1}}{\partial x_{l_{6}}}=x_{1} \frac{\partial \mu}{\partial x_{l_{6}}}, \frac{\partial f_{1}}{\partial x_{l_{2}}}=\frac{\partial f_{1}}{\partial x_{3}}=0, \frac{\partial f_{2}}{\partial x_{1}}=-q_{2}, \frac{\partial f_{2}}{\partial x_{l_{1}}}=-\frac{x_{1}}{u_{4}} \frac{\partial \mu}{\partial x_{l_{1}}}, \\
& \frac{\partial f_{2}}{\partial x_{l_{2}}}=0, \frac{\partial f_{l_{3}}}{\partial x_{1}}=q_{l_{3}}, \frac{\partial f_{l_{3}}}{\partial x_{l_{6}}}=x_{1} \frac{\partial q_{l_{3}}}{\partial x_{l_{6}}}, \frac{\partial f_{l_{3}}}{\partial x_{3}\left(t-\tau_{3}\right)}=x_{1} \frac{\partial q_{l_{3}}}{\partial x_{3}\left(t-\tau_{3}\right)}, \frac{\partial f_{l_{3}}}{\partial x_{l_{2}}}=0, \frac{\partial f_{l_{3}}}{\partial x_{3}}=0, \frac{\partial f_{6}}{\partial x_{1}}=0, \\
& \frac{\partial f_{6}}{\partial x_{2}}=\frac{1}{u_{19}}\left[\frac{u_{20}}{x_{2}+u_{21}}-\frac{u_{20} x_{2}}{x_{2}+u_{21}}+\frac{1}{u_{22}}-\frac{\partial q_{2}}{\partial x_{2}}\right]-x_{6}\left(t-\tau_{6}\right) \frac{\partial \mu}{\partial x_{2}}, \frac{\partial f_{6}}{\partial x_{3}\left(t-\tau_{3}\right)}=-x_{6} \frac{\partial \mu}{\partial x_{3}\left(t-\tau_{3}\right)}, \frac{\partial f_{6}}{\partial x_{4}}=-x_{6}\left(t-\tau_{6}\right) \frac{\partial \mu}{\partial x_{4}}, \\
& \frac{\partial f_{6}}{\partial x_{5}}=-x_{6}\left(t-\tau_{6}\right) \frac{\partial \mu}{\partial x_{5}}, \frac{\partial f_{6}}{\partial x_{l_{2}}}=0, \frac{\partial f_{7}}{\partial x_{1}}=\frac{\partial f_{7}}{\partial x_{l_{2}}}=0, \frac{\partial f_{7}}{\partial x_{14}}=-\frac{u_{26} U_{P} x_{7}\left(t-\tau_{7}\right)}{u_{27}+x_{7}\left(t-\tau_{7}\right)\left(1+\frac{x_{7}\left(t-\tau_{7}\right)}{u_{28}}\right)}, \\
& \frac{\partial f_{7}}{\partial x_{3}\left(t-\tau_{3}\right)}=-x_{7}\left(t-\tau_{7}\right) \frac{\partial \mu}{\partial x_{3}\left(t-\tau_{3}\right)}+\frac{\partial U_{G}}{\partial x_{3}\left(t-\tau_{3}\right)} \frac{u_{23} x_{6}\left(t-\tau_{6}\right) x_{12}}{u_{24}\left(1+\frac{x_{7}\left(t-\tau_{7}\right)}{u_{25}}\right)+x_{6}\left(t-\tau_{6}\right)}
\end{aligned}
$$$$
-\frac{\partial U_{P}}{\partial x_{3}\left(t-\tau_{3}\right)} \frac{u_{26} x_{7}\left(t-\tau_{7}\right) x_{14}}{u_{27}+x_{7}\left(t-\tau_{7}\right)\left(1+\frac{x_{7}\left(t-\tau_{7}\right)}{u_{28}}\right)},
$$$$
\frac{\partial f_{7}}{\partial x_{l_{6}}}=-x_{7}\left(t-\tau_{7}\right) \frac{\partial \mu}{\partial x_{l_{6}}}+\frac{\partial U_{G}}{\partial x_{l_{6}}} \frac{u_{23} x_{6}\left(t-\tau_{6}\right) x_{12}}{u_{24}\left(1+\frac{x_{7}\left(t-\tau_{7}\right)}{u_{25}}\right)+x_{6}\left(t-\tau_{6}\right)}-\frac{\partial U_{P}}{\partial x_{l_{6}}} \frac{u_{26} x_{7}\left(t-\tau_{7}\right) x_{14}}{u_{27}+x_{7}\left(t-\tau_{7}\right)\left(1+\frac{x_{7}\left(t-\tau_{7}\right)}{u_{28}}\right)} \text {, }
$$$$
\frac{\partial f_{8}}{\partial x_{1}}=\frac{\partial f_{8}}{\partial x_{6}}=\frac{\partial f_{8}}{\partial x_{7}}=0, \frac{\partial f_{8}}{\partial x_{l_{6}}}=-x_{8}\left(t-\tau_{8}\right) \frac{\partial \mu}{\partial x_{l_{6}}}+\frac{\partial U_{P}}{\partial x_{l_{6}}} \frac{u_{23} u_{26} x_{7}\left(t-\tau_{7}\right) x_{14}}{u_{27}+x_{7}\left(t-\tau_{7}\right)\left(1+\frac{x_{7}\left(t-\tau_{7}\right)}{u_{28}}\right)},
$$$$
\frac{\partial f_{8}}{\partial x_{3}\left(t-\tau_{3}\right)}=u_{33}-x_{8}\left(t-\tau_{8}\right) \frac{\partial \mu}{\partial x_{3}\left(t-\tau_{3}\right)}+\frac{\partial U_{P}}{\partial x_{3}\left(t-\tau_{3}\right)} \frac{u_{23} u_{26} x_{7}\left(t-\tau_{7}\right) x_{14}}{u_{27}+x_{7}\left(t-\tau_{7}\right)\left(1+\frac{x_{7}\left(t-\tau_{7}\right)}{u_{28}}\right)},
$$$$
\frac{\partial f_{8}}{\partial x_{l_{5}}}=0, \frac{\partial f_{8}}{\partial x_{14}}=\frac{u_{23} u_{26} U_{P} x_{7}}{u_{27}+x_{7}\left(t-\tau_{7}\right)\left(1+\frac{x_{7}\left(t-\tau_{7}\right)}{u_{28}}\right)}, \frac{\partial f_{9}}{\partial x_{1}}=\frac{\partial f_{9}}{\partial x_{6}}=\frac{\partial f_{9}}{\partial x_{7}}=\frac{\partial f_{9}}{\partial x_{8}}=0, \frac{\partial f_{9}}{\partial x_{l_{6}}}=-x_{9} \frac{\partial \mu}{\partial x_{l_{6}}},
$$

$\frac{\partial f_{l_{15}}}{\partial x_{3}\left(t-\tau_{3}\right)}=-x_{l_{15}} \frac{\partial \mu}{\partial x_{3}\left(t-\tau_{3}\right)}, \frac{\partial f_{9}}{\partial x_{9}}=-\left(u_{35}+\mu\right), \frac{\partial f_{9}}{\partial x_{l_{8}}}=0, \frac{\partial f_{10}}{\partial x_{1}}=\frac{\partial f_{10}}{\partial x_{6}}=0$,

$\frac{\partial f_{10}}{\partial x_{l_{6}}}=-x_{10} \frac{\partial \mu}{\partial x_{l_{6}}}, \frac{\partial f_{10}}{\partial x_{7}}=\frac{\partial f_{10}}{\partial x_{8}}=0, \frac{\partial f_{10}}{\partial x_{9}}=u_{36}, \frac{\partial f_{10}}{\partial x_{10}}=-\left(u_{39}+\mu\right), \frac{\partial f_{10}}{\partial x_{l_{9}}}=0$,

$\frac{\partial f_{11}}{\partial x_{1}}=\frac{\partial f_{11}}{\partial x_{6}}=\frac{\partial f_{11}}{\partial x_{7}}=\frac{\partial f_{11}}{\partial x_{l_{10}}}=\frac{\partial f_{11}}{\partial x_{l_{11}}}=0, \frac{\partial f_{11}}{\partial x_{l_{6}}}=-x_{11} \frac{\partial \mu}{\partial x_{l_{6}}}, \frac{\partial f_{11}}{\partial x_{11}}=-\left(u_{40}+\mu\right)$,

$\frac{\partial f_{12}}{\partial x_{1}}=\frac{\partial f_{12}}{\partial x_{3}}=\frac{\partial f_{12}}{\partial x_{12}}=\frac{\partial f_{12}}{\partial x_{13}}=\frac{\partial f_{12}}{\partial x_{14}}=0, \frac{\partial f_{12}}{\partial x_{l_{6}}}=-x_{12} \frac{\partial \mu}{\partial x_{l_{6}}}, \frac{\partial f_{12}}{\partial x_{11}}=u_{41}$,

$\frac{\partial f_{12}}{\partial x_{12}}=-\left(u_{42}+\mu\right), \frac{\partial f_{13}}{\partial x_{1}}=\frac{\partial f_{13}}{\partial x_{3}}=\frac{\partial f_{13}}{\partial x_{6}}=\frac{\partial f_{13}}{\partial x_{7}}=\frac{\partial f_{13}}{\partial x_{l_{13}}}=\frac{\partial f_{13}}{\partial x_{14}}=0, \frac{\partial f_{13}}{\partial x_{l_{6}}}=-x_{13} \frac{\partial \mu}{\partial x_{16}}$,

$\frac{\partial f_{13}}{\partial x_{13}}=-\left(u_{43}+\mu\right), \frac{\partial f_{14}}{\partial x_{1}}=\frac{\partial f_{14}}{\partial x_{l_{3}}}=\frac{\partial f_{14}}{\partial x_{l_{14}}}=0, \frac{\partial f_{14}}{\partial x_{l_{6}}}=-x_{14} \frac{\partial \mu}{\partial x_{l_{6}}}, \frac{\partial f_{14}}{\partial x_{14}}=u_{44}-u_{45}-\mu$.

The explicit formulas for $\frac{\partial f\left(x(t \mid \tau, u), x_{\tau}, u\right)}{\partial x\left(t-\tau_{6} \mid \tau, u\right)}$ in (30), denoted by $\frac{\partial f}{\partial x\left(t-\tau_{6}\right)}$ are given below. For $l_{1}=2, \ldots, 5, l_{2}=$ $6, \ldots, 14, l_{3}=3, \ldots, 5, l_{4}=7, \ldots, 14, l_{5}=8, \ldots, 13, l_{6}=2,4,5, l_{7}=9, \ldots, 13, l_{8}=10, \ldots, 14, l_{9}=11, \ldots, 14, l_{10}=$ 
$8, \ldots, 10, l_{11}=12, \ldots, 14, l_{12}=6, \ldots, 10, l_{13}=8, \ldots, 12, l_{14}=6, \ldots, 13$,

$$
\begin{aligned}
& \frac{\partial f_{1}}{\partial x_{1}}=\mu, \frac{\partial f_{1}}{\partial x_{l_{6}}}=x_{1} \frac{\partial \mu}{\partial x_{l_{6}}}, \frac{\partial f_{1}}{\partial x_{l_{2}}}=\frac{\partial f_{1}}{\partial x_{3}}=0, \frac{\partial f_{2}}{\partial x_{1}}=-q_{2}, \frac{\partial f_{2}}{\partial x_{l_{1}}}=-\frac{x_{1}}{u_{4}} \frac{\partial \mu}{\partial x_{l_{1}}}, \frac{\partial f_{2}}{\partial x_{l_{2}}}=0, \frac{\partial f_{l_{3}}}{\partial x_{1}}=q_{l_{3}}, \frac{\partial f_{l_{3}}}{\partial x_{l_{6}}}=x_{1} \frac{\partial q_{l_{3}}}{\partial x_{l_{6}}}, \frac{\partial f_{l_{3}}}{\partial x_{l_{2}}}=0, \\
& \frac{\partial f_{l_{3}}}{\partial x_{3}}=0, \frac{\partial f_{6}}{\partial x_{1}}=0, \frac{\partial f_{6}}{\partial x_{2}}=\frac{1}{u_{19}}\left[\frac{u_{20}}{x_{2}+u_{21}}-\frac{u_{20} x_{2}}{x_{2}+u_{21}}+\frac{1}{u_{22}}-\frac{\partial q_{2}}{\partial x_{2}}\right]-x_{6}\left(t-\tau_{6}\right) \frac{\partial \mu}{\partial x_{2}}, \frac{\partial f_{6}}{\partial x_{4}}=-x_{6}\left(t-\tau_{6}\right) \frac{\partial \mu}{\partial x_{4}}, \\
& \frac{\partial f_{6}}{\partial x_{5}}=-x_{6}\left(t-\tau_{6}\right) \frac{\partial \mu}{\partial x_{5}}, \frac{\partial f_{6}}{\partial x_{3}}=\frac{\partial f_{6}}{\partial x_{l_{6}}}=0, \frac{\partial f_{6}}{\partial x_{6}\left(t-\tau_{6}\right)}=-\frac{1}{u_{22}}-\mu, \frac{\partial f_{7}}{\partial x_{1}}=\frac{\partial f_{7}}{\partial x_{3}}=\frac{\partial f_{7}}{\partial x_{l_{4}}}=0 \text {, } \\
& \frac{\partial f_{7}}{\partial x_{14}}=-\frac{u_{26} U_{P} x_{7}\left(t-\tau_{7}\right)}{u_{27}+x_{7}\left(t-\tau_{7}\right)\left(1+\frac{x_{7}\left(t-\tau_{7}\right)}{u_{28}}\right)}, \frac{\partial f_{7}}{\partial x_{6}\left(t-\tau_{6}\right)}=\frac{u_{23} U_{G} x_{12}}{\sigma_{24}\left(1+\frac{x_{7}\left(t-\tau_{7}\right)}{u_{25}}\right)+x_{6}\left(t-\tau_{6}\right)}-\frac{u_{23} U_{G} x_{6}\left(t-\tau_{6}\right) x_{12}}{\left[u_{24}\left(1+\frac{x_{7}\left(t-\tau_{7}\right)}{u_{25}}\right)+x_{6}\left(t-\tau_{6}\right)\right]^{2}}, \\
& \frac{\partial f_{7}}{\partial x_{l_{6}}}=-x_{7}\left(t-\tau_{7}\right) \frac{\partial \mu}{\partial x_{l_{6}}}+\frac{\partial U_{G}}{\partial x_{l_{6}}} \frac{u_{23} x_{6}\left(t-\tau_{6}\right) x_{12}}{u_{24}\left(1+\frac{x_{7}}{u_{25}}\right)+x_{6}\left(t-\tau_{6}\right)}-\frac{\partial U_{P}}{\partial x_{l_{6}}} \frac{u_{26} x_{7}\left(t-\tau_{7}\right) x_{14}}{u_{27}+x_{7}\left(t-\tau_{7}\right)\left(1+\frac{x_{7}\left(t-\tau_{7}\right)}{u_{28}}\right)}, \\
& \frac{\partial f_{8}}{\partial x_{1}}=\frac{\partial f_{8}}{\partial x_{3}}=\frac{\partial f_{8}}{\partial x_{6}}=\frac{\partial f_{8}}{\partial x_{7}}=0, \frac{\partial f_{8}}{\partial x_{l_{6}}}=-x_{8}\left(t-\tau_{8}\right) \frac{\partial \mu}{\partial x_{l_{6}}}+\frac{\partial U_{P}}{\partial x_{l_{6}}} \frac{u_{23} u_{26} x_{7}\left(t-\tau_{7}\right) x_{14}}{u_{27}+x_{7}\left(t-\tau_{7}\right)\left(1+\frac{x_{7}\left(t-\tau_{7}\right)}{u_{28}}\right)}, \\
& \frac{\partial f_{8}}{\partial x_{l_{5}}}=0, \frac{\partial f_{8}}{\partial x_{14}}=\frac{u_{23} u_{26} U_{P} x_{7}}{u_{27}+x_{7}\left(t-\tau_{7}\right)\left(1+\frac{x_{7}\left(t-\tau_{7}\right)}{u_{28}}\right)}, \frac{\partial f_{9}}{\partial x_{1}}=\frac{\partial f_{9}}{\partial x_{3}}=\frac{\partial f_{9}}{\partial x_{6}}=\frac{\partial f_{9}}{\partial x_{7}}=\frac{\partial f_{9}}{\partial x_{8}}=0, \frac{\partial f_{9}}{\partial x_{l_{6}}}=-x_{9} \frac{\partial \mu}{\partial x_{l_{6}}} \\
& \frac{\partial f_{9}}{\partial x_{9}}=-\left(u_{35}+\mu\right), \frac{\partial f_{9}}{\partial x_{l_{8}}}=0, \frac{\partial f_{10}}{\partial x_{1}}=\frac{\partial f_{10}}{\partial x_{3}}=\frac{\partial f_{10}}{\partial x_{6}}=0 \\
& \frac{\partial f_{10}}{\partial x_{l_{6}}}=-x_{10} \frac{\partial \mu}{\partial x_{l_{6}}}, \frac{\partial f_{10}}{\partial x_{7}}=\frac{\partial f_{10}}{\partial x_{8}}=0, \frac{\partial f_{10}}{\partial x_{9}}=u_{36}, \frac{\partial f_{10}}{\partial x_{10}}=-\left(u_{39}+\mu\right), \frac{\partial f_{10}}{\partial x_{l_{9}}}=0, \\
& \frac{\partial f_{11}}{\partial x_{1}}=\frac{\partial f_{11}}{\partial x_{3}}=\frac{\partial f_{11}}{\partial x_{6}}=\frac{\partial f_{11}}{\partial x_{7}}=\frac{\partial f_{11}}{\partial x_{l_{10}}}=\frac{\partial f_{11}}{\partial x_{l_{11}}}=0, \frac{\partial f_{11}}{\partial x_{l_{6}}}=-x_{11} \frac{\partial \mu}{\partial x_{l_{6}}}, \frac{\partial f_{11}}{\partial x_{11}}=-\left(u_{40}+\mu\right), \\
& \frac{\partial f_{12}}{\partial x_{1}}=\frac{\partial f_{12}}{\partial x_{3}}=\frac{\partial f_{12}}{\partial x_{l_{12}}}=\frac{\partial f_{12}}{\partial x_{13}}=\frac{\partial f_{12}}{\partial x_{14}}=0, \frac{\partial f_{12}}{\partial x_{l_{6}}}=-x_{12} \frac{\partial \mu}{\partial x_{l_{6}}}, \frac{\partial f_{12}}{\partial x_{11}}=u_{41}, \\
& \frac{\partial f_{12}}{\partial x_{12}}=-\left(u_{42}+\mu\right), \frac{\partial f_{13}}{\partial x_{1}}=\frac{\partial f_{13}}{\partial x_{3}}=\frac{\partial f_{13}}{\partial x_{6}}=\frac{\partial f_{13}}{\partial x_{7}}=\frac{\partial f_{13}}{\partial x_{l_{13}}}=\frac{\partial f_{13}}{\partial x_{14}}=0, \frac{\partial f_{13}}{\partial x_{l_{6}}}=-x_{13} \frac{\partial \mu}{\partial x_{l_{6}}}, \\
& \frac{\partial f_{13}}{\partial x_{13}}=-\left(u_{43}+\mu\right), \frac{\partial f_{14}}{\partial x_{1}}=\frac{\partial f_{14}}{\partial x_{l_{3}}}=\frac{\partial f_{14}}{\partial x_{l_{14}}}=0, \frac{\partial f_{14}}{\partial x_{l_{6}}}=-x_{14} \frac{\partial \mu}{\partial x_{l_{6}}}, \frac{\partial f_{14}}{\partial x_{14}}=u_{44}-u_{45}-\mu \text {. }
\end{aligned}
$$

The explicit formulas for $\frac{\partial f\left(x(t \mid \tau, u), x_{\tau}, u\right)}{\partial x\left(t-\tau_{7} \mid \tau, u\right)}$ in (30), denoted by $\frac{\partial f}{\partial x\left(t-\tau_{7}\right)}$ are given below. For $l_{1}=2, \ldots, 5, l_{2}=$ $6, \ldots, 14, l_{3}=3, \ldots, 5, l_{4}=7, \ldots, 14, l_{5}=8, \ldots, 13, l_{6}=2,4,5, l_{7}=9, \ldots, 13, l_{8}=10, \ldots, 14, l_{9}=11, \ldots, 14, l_{10}=$ 
$8, \ldots, 10, l_{11}=12, \ldots, 14, l_{12}=6, \ldots, 10, l_{13}=8, \ldots, 12, l_{14}=6, \ldots, 13, l_{14}=8, \ldots, 14$

$$
\begin{aligned}
& \frac{\partial f_{1}}{\partial x_{1}}=\mu, \frac{\partial f_{1}}{\partial x_{l_{6}}}=x_{1} \frac{\partial \mu}{\partial x_{l_{6}}}, \frac{\partial f_{1}}{\partial x_{l_{2}}}=\frac{\partial f_{1}}{\partial x_{3}}=0, \frac{\partial f_{2}}{\partial x_{1}}=-q_{2}, \frac{\partial f_{2}}{\partial x_{l_{1}}}=-\frac{x_{1}}{u_{4}} \frac{\partial \mu}{\partial x_{l_{1}}}, \frac{\partial f_{2}}{\partial x_{l_{2}}}=0, \frac{\partial f_{l_{3}}}{\partial x_{1}}=q_{l_{3}}, \frac{\partial f_{l_{3}}}{\partial x_{l_{6}}}=x_{1} \frac{\partial q_{l_{3}}}{\partial x_{l_{6}}}, \frac{\partial f_{l_{3}}}{\partial x_{l_{2}}}=0, \\
& \frac{\partial f_{l_{3}}}{\partial x_{3}}=0, \frac{\partial f_{6}}{\partial x_{1}}=0, \frac{\partial f_{6}}{\partial x_{2}}=\frac{1}{u_{19}}\left[\frac{u_{20}}{x_{2}+u_{21}}-\frac{u_{20} x_{2}}{x_{2}+u_{21}}+\frac{1}{u_{22}}-\frac{\partial q_{2}}{\partial x_{2}}\right]-x_{6}\left(t-\tau_{6}\right) \frac{\partial \mu}{\partial x_{2}}, \frac{\partial f_{6}}{\partial x_{4}}=-x_{6}\left(t-\tau_{6}\right) \frac{\partial \mu}{\partial x_{4}}, \\
& \frac{\partial f_{6}}{\partial x_{5}}=-x_{6}\left(t-\tau_{6}\right) \frac{\partial \mu}{\partial x_{5}}, \frac{\partial f_{6}}{\partial x_{3}}=\frac{\partial f_{6}}{\partial x_{l_{2}}}=0, \frac{\partial f_{7}}{\partial x_{1}}=\frac{\partial f_{7}}{\partial x_{3}}=\frac{\partial f_{7}}{\partial x_{6}}=\frac{\partial f_{7}}{\partial x_{l_{15}}}=0, \frac{\partial f_{7}}{\partial x_{14}}=-\frac{u_{26} U_{P} x_{7}\left(t-\tau_{7}\right)}{u_{27}+x_{7}\left(t-\tau_{7}\right)\left(1+\frac{x_{7}\left(t-\tau_{7}\right)}{u_{28}}\right)}, \\
& \frac{\partial f_{7}}{\partial x_{7}\left(t-\tau_{7}\right)}=-\frac{u_{23} U_{G} x_{6}\left(t-\tau_{6}\right) x_{12}}{\left[u_{24}\left(1+\frac{x_{7}\left(t-\tau_{7}\right)}{u_{25}}\right)+x_{6}\left(t-\tau_{6}\right)\right]^{2}} \frac{u_{24}}{u_{25}}-\frac{u_{26} U_{P} x_{14}}{u_{27}+x_{7}\left(t-\tau_{7}\right)\left(1+\frac{x_{7}\left(t-\tau_{7}\right)}{u_{28}}\right)} \\
& +\frac{u_{26} U_{P} x_{7}\left(t-\tau_{7}\right) x_{14}\left(1+\frac{2 x_{7}\left(t-\tau_{7}\right)}{u_{28}}\right)}{\left[u_{27}+x_{7}\left(t-\tau_{7}\right)\left(1+\frac{x_{7}\left(t-\tau_{7}\right)}{u_{28}}\right)\right]^{2}}-\frac{u_{29}}{x_{7}\left(t-\tau_{7}\right)+u_{30}}+\frac{u_{29} x_{7}\left(t-\tau_{7}\right)}{\left(x_{7}\left(t-\tau_{7}\right)+u_{30}\right)^{2}}-\mu, \\
& \frac{\partial f_{7}}{\partial x_{l_{6}}}=-x_{7}\left(t-\tau_{7}\right) \frac{\partial \mu}{\partial x_{l_{6}}}+\frac{\partial U_{G}}{\partial x_{l_{6}}} \frac{u_{23} x_{6}\left(t-\tau_{6}\right) x_{12}}{u_{24}\left(1+\frac{x_{7}}{u_{25}}\right)+x_{6}\left(t-\tau_{6}\right)}-\frac{\partial U_{P}}{\partial x_{l_{6}}} \frac{u_{26} x_{7}\left(t-\tau_{7}\right) x_{14}}{u_{27}+x_{7}\left(t-\tau_{7}\right)\left(1+\frac{x_{7}\left(t-\tau_{7}\right)}{u_{28}}\right)}, \\
& \frac{\partial f_{8}}{\partial x_{1}}=\frac{\partial f_{8}}{\partial x_{3}}=\frac{\partial f_{8}}{\partial x_{6}}=0, \frac{\partial f_{8}}{\partial x_{l_{6}}}=-x_{8}\left(t-\tau_{8}\right) \frac{\partial \mu}{\partial x_{l_{6}}}+\frac{\partial U_{P}}{\partial x_{l_{6}}} \frac{u_{23} u_{26} x_{7}\left(t-\tau_{7}\right) x_{14}}{u_{27}+x_{7}\left(t-\tau_{7}\right)\left(1+\frac{x_{7}\left(t-\tau_{7}\right)}{u_{28}}\right)} \\
& \frac{\partial f_{8}}{\partial x_{7}\left(t-\tau_{7}\right)}=\frac{u_{23} u_{26} U_{P} x_{14}}{u_{27}+x_{7}\left(t-\tau_{7}\right)\left(1+\frac{x_{7}\left(t-\tau_{7}\right)}{u_{28}}\right)}-\frac{u_{23} u_{26} U_{P} x_{7}\left(t-\tau_{7}\right) x_{14}\left(1+\frac{2 x_{7}\left(t-\tau_{7}\right)}{u_{28}}\right)}{\left[u_{27}+x_{7}\left(t-\tau_{7}\right)\left(1+\frac{x_{7}\left(t-\tau_{7}\right)}{u_{28}}\right)\right]^{2}} \\
& \frac{\partial f_{8}}{\partial x_{l_{5}}}=0, \frac{\partial f_{8}}{\partial x_{14}}=\frac{u_{23} u_{26} U_{P} x_{7}}{u_{27}+x_{7}\left(t-\tau_{7}\right)\left(1+\frac{x_{7}\left(t-\tau_{7}\right)}{u_{28}}\right)}, \frac{\partial f_{9}}{\partial x_{1}}=\frac{\partial f_{9}}{\partial x_{3}}=\frac{\partial f_{9}}{\partial x_{6}}=\frac{\partial f_{9}}{\partial x_{8}}=0, \frac{\partial f_{9}}{\partial x_{l_{6}}}=-x_{9} \frac{\partial \mu}{\partial x_{l_{6}}} \\
& \frac{\partial f_{9}}{\partial x_{9}}=-\left(u_{35}+\mu\right), \frac{\partial f_{9}}{\partial x_{l_{8}}}=0, \frac{\partial f_{9}}{\partial x_{7}\left(t-\tau_{7}\right)}=\frac{1}{x_{7}\left(t-\tau_{7}\right)+u_{30}+u_{34} x_{7}\left(t-\tau_{7}\right)}-\frac{\left(x_{7}\left(t-\tau_{7}\right)+u_{30}\right)\left(1+u_{34}\right)}{\left(x_{7}\left(t-\tau_{7}\right)+u_{30}+u_{34} x_{7}\left(t-\tau_{7}\right)\right)^{2}}, \\
& \frac{\partial f_{10}}{\partial x_{1}}=\frac{\partial f_{10}}{\partial x_{3}}=\frac{\partial f_{10}}{\partial x_{6}}=0, \frac{\partial f_{10}}{\partial x_{l_{6}}}=-x_{10} \frac{\partial \mu}{\partial x_{l_{6}}}, \frac{\partial f_{10}}{\partial x_{8}}=0, \frac{\partial f_{10}}{\partial x_{9}}=u_{36}, \frac{\partial f_{10}}{\partial x_{10}}=-\left(u_{39}+\mu\right), \frac{\partial f_{10}}{\partial x_{l_{9}}}=0 \text {, } \\
& \frac{\partial f_{10}}{\partial x_{7}\left(t-\tau_{7}\right)}=-\frac{u_{37} u_{38}}{x_{7}\left(t-\tau_{7}\right)+u_{30}}+\frac{u_{37} u_{38} x_{7}\left(t-\tau_{7}\right)}{\left(x_{7}\left(t-\tau_{7}\right)+u_{30}\right)^{2}}, \frac{\partial f_{13}}{\partial x_{7}\left(t-\tau_{7}\right)}=\frac{\partial f_{11}}{\partial x_{7}\left(t-\tau_{7}\right)}=\frac{\partial f_{9}}{\partial x_{7}\left(t-\tau_{7}\right)}, \\
& \frac{\partial f_{11}}{\partial x_{1}}=\frac{\partial f_{11}}{\partial x_{3}}=\frac{\partial f_{11}}{\partial x_{6}}=\frac{\partial f_{11}}{\partial x_{l_{10}}}=\frac{\partial f_{11}}{\partial x_{l_{11}}}=0, \frac{\partial f_{11}}{\partial x_{l_{6}}}=-x_{11} \frac{\partial \mu}{\partial x_{l_{6}}}, \frac{\partial f_{11}}{\partial x_{11}}=-\left(u_{40}+\mu\right) \text {, } \\
& \frac{\partial f_{12}}{\partial x_{1}}=\frac{\partial f_{12}}{\partial x_{3}}=\frac{\partial f_{12}}{\partial x_{l_{12}}}=\frac{\partial f_{12}}{\partial x_{13}}=\frac{\partial f_{12}}{\partial x_{14}}=0, \frac{\partial f_{12}}{\partial x_{l_{6}}}=-x_{12} \frac{\partial \mu}{\partial x_{l_{6}}}, \frac{\partial f_{12}}{\partial x_{11}}=u_{41} \text {, } \\
& \frac{\partial f_{12}}{\partial x_{12}}=-\left(u_{42}+\mu\right), \frac{\partial f_{13}}{\partial x_{1}}=\frac{\partial f_{13}}{\partial x_{3}}=\frac{\partial f_{13}}{\partial x_{6}}=\frac{\partial f_{13}}{\partial x_{7}}=\frac{\partial f_{13}}{\partial x_{l_{13}}}=\frac{\partial f_{13}}{\partial x_{14}}=0, \frac{\partial f_{13}}{\partial x_{l_{6}}}=-x_{13} \frac{\partial \mu}{\partial x_{l_{6}}}, \\
& \frac{\partial f_{13}}{\partial x_{13}}=-\left(u_{43}+\mu\right), \frac{\partial f_{14}}{\partial x_{1}}=\frac{\partial f_{14}}{\partial x_{l_{3}}}=\frac{\partial f_{14}}{\partial x_{l_{14}}}=0, \frac{\partial f_{14}}{\partial x_{l_{6}}}=-x_{14} \frac{\partial \mu}{\partial x_{l_{6}}}, \frac{\partial f_{14}}{\partial x_{14}}=u_{44}-u_{45}-\mu \text {. }
\end{aligned}
$$

The explicit formulas for $\frac{\partial f\left(x(t \mid \tau, u), x_{\tau}, u\right)}{\partial x\left(t-\tau_{8} \mid \tau, u\right)}$ in (30), denoted by $\frac{\partial f}{\partial x\left(t-\tau_{8}\right)}$ are given below. For $l_{1}=2, \ldots, 5, l_{2}=$ $6, \ldots, 14, l_{3}=3, \ldots, 5, l_{4}=7, \ldots, 14, l_{5}=8, \ldots, 13, l_{6}=2,4,5, l_{7}=9, \ldots, 13, l_{8}=10, \ldots, 14, l_{9}=11, \ldots, 14, l_{10}=$ 
$8, \ldots, 10, l_{11}=12, \ldots, 14, l_{12}=6, \ldots, 10, l_{13}=8, \ldots, 12, l_{14}=6, \ldots, 13$

$$
\begin{aligned}
& \frac{\partial f_{1}}{\partial x_{1}}=\mu, \frac{\partial f_{1}}{\partial x_{l_{6}}}=x_{1} \frac{\partial \mu}{\partial x_{l_{6}}}, \frac{\partial f_{1}}{\partial x_{l_{2}}}=\frac{\partial f_{1}}{\partial x_{3}}=0, \frac{\partial f_{2}}{\partial x_{1}}=-q_{2}, \frac{\partial f_{2}}{\partial x_{l_{1}}}=-\frac{x_{1}}{u_{4}} \frac{\partial \mu}{\partial x_{l_{1}}}, \frac{\partial f_{2}}{\partial x_{l_{2}}}=0, \frac{\partial f_{l_{3}}}{\partial x_{1}}=q_{l_{3}}, \frac{\partial f_{l_{3}}}{\partial x_{l_{6}}}=x_{1} \frac{\partial q_{l_{3}}}{\partial x_{l_{6}}}, \frac{\partial f_{l_{3}}}{\partial x_{l_{2}}}=0, \\
& \frac{\partial f_{l_{3}}}{\partial x_{3}}=0, \frac{\partial f_{6}}{\partial x_{1}}=0, \frac{\partial f_{6}}{\partial x_{2}}=\frac{1}{u_{19}}\left[\frac{u_{20}}{x_{2}+u_{21}}-\frac{u_{20} x_{2}}{x_{2}+u_{21}}+\frac{1}{u_{22}}-\frac{\partial q_{2}}{\partial x_{2}}\right]-x_{6}\left(t-\tau_{6}\right) \frac{\partial \mu}{\partial x_{2}}, \frac{\partial f_{6}}{\partial x_{4}}=-x_{6}\left(t-\tau_{6}\right) \frac{\partial \mu}{\partial x_{4}}, \\
& \frac{\partial f_{6}}{\partial x_{5}}=-x_{6}\left(t-\tau_{6}\right) \frac{\partial \mu}{\partial x_{5}}, \frac{\partial f_{6}}{\partial x_{3}}=\frac{\partial f_{6}}{\partial x_{l_{2}}}=0, \frac{\partial f_{7}}{\partial x_{1}}=\frac{\partial f_{7}}{\partial x_{3}}=\frac{\partial f_{7}}{\partial x_{l_{2}}}=0, \frac{\partial f_{7}}{\partial x_{14}}=-\frac{u_{26} U_{P} x_{7}\left(t-\tau_{7}\right)}{u_{27}+x_{7}\left(t-\tau_{7}\right)\left(1+\frac{x_{7}\left(t-\tau_{7}\right)}{u_{28}}\right)} \text {, } \\
& \frac{\partial f_{7}}{\partial x_{l_{6}}}=-x_{7}\left(t-\tau_{7}\right) \frac{\partial \mu}{\partial x_{l_{6}}}+\frac{\partial U_{G}}{\partial x_{l_{6}}} \frac{u_{23} x_{6}\left(t-\tau_{6}\right) x_{12}}{u_{24}\left(1+\frac{x_{7}}{u_{25}}\right)+x_{6}\left(t-\tau_{6}\right)}-\frac{\partial U_{P}}{\partial x_{l_{6}}} \frac{u_{26} x_{7}\left(t-\tau_{7}\right) x_{14}}{u_{27}+x_{7}\left(t-\tau_{7}\right)\left(1+\frac{x_{7}\left(t-\tau_{7}\right)}{u_{28}}\right)}, \\
& \frac{\partial f_{8}}{\partial x_{1}}=\frac{\partial f_{8}}{\partial x_{3}}=\frac{\partial f_{8}}{\partial x_{6}}=\frac{\partial f_{8}}{\partial x_{7}}=0, \frac{\partial f_{8}}{\partial x_{l_{6}}}=-x_{8}\left(t-\tau_{8}\right) \frac{\partial \mu}{\partial x_{l_{6}}}+\frac{\partial U_{P}}{\partial x_{l_{6}}} \frac{u_{23} u_{26} x_{7}\left(t-\tau_{7}\right) x_{14}}{u_{27}+x_{7}\left(t-\tau_{7}\right)\left(1+\frac{x_{7}\left(t-\tau_{7}\right)}{u_{28}}\right)}, \\
& \frac{\partial f_{8}}{\partial x_{8}\left(t-\tau_{8}\right)}=\frac{-u_{31}}{x_{8}\left(t-\tau_{8}\right)+u_{32}}+\frac{u_{31} x_{8}\left(t-\tau_{8}\right)}{\left(x_{8}\left(t-\tau_{8}\right)+u_{32}\right)^{2}}-u_{33}-\mu \\
& \frac{\partial f_{8}}{\partial x_{l_{5}}}=0, \frac{\partial f_{8}}{\partial x_{14}}=\frac{u_{23} u_{26} U_{P} x_{7}}{u_{27}+x_{7}\left(t-\tau_{7}\right)\left(1+\frac{x_{7}\left(t-\tau_{7}\right)}{u_{28}}\right)}, \frac{\partial f_{9}}{\partial x_{1}}=\frac{\partial f_{9}}{\partial x_{3}}=\frac{\partial f_{9}}{\partial x_{6}}=\frac{\partial f_{9}}{\partial x_{7}}=\frac{\partial f_{9}}{\partial x_{8}}=0, \frac{\partial f_{9}}{\partial x_{l_{6}}}=-x_{9} \frac{\partial \mu}{\partial x_{l_{6}}} \\
& \frac{\partial f_{9}}{\partial x_{9}}=-\left(u_{35}+\mu\right), \frac{\partial f_{9}}{\partial x_{l_{8}}}=0, \frac{\partial f_{10}}{\partial x_{1}}=\frac{\partial f_{10}}{\partial x_{3}}=\frac{\partial f_{10}}{\partial x_{6}}=0 \\
& \frac{\partial f_{10}}{\partial x_{l_{6}}}=-x_{10} \frac{\partial \mu}{\partial x_{l_{6}}}, \frac{\partial f_{10}}{\partial x_{7}}=\frac{\partial f_{10}}{\partial x_{8}}=0, \frac{\partial f_{10}}{\partial x_{9}}=u_{36}, \frac{\partial f_{10}}{\partial x_{10}}=-\left(u_{39}+\mu\right), \frac{\partial f_{10}}{\partial x_{l_{9}}}=0, \\
& \frac{\partial f_{11}}{\partial x_{1}}=\frac{\partial f_{11}}{\partial x_{3}}=\frac{\partial f_{11}}{\partial x_{6}}=\frac{\partial f_{11}}{\partial x_{7}}=\frac{\partial f_{11}}{\partial x_{l_{10}}}=\frac{\partial f_{11}}{\partial x_{l_{11}}}=0, \frac{\partial f_{11}}{\partial x_{l_{6}}}=-x_{11} \frac{\partial \mu}{\partial x_{l_{6}}}, \frac{\partial f_{11}}{\partial x_{11}}=-\left(u_{40}+\mu\right), \\
& \frac{\partial f_{12}}{\partial x_{1}}=\frac{\partial f_{12}}{\partial x_{3}}=\frac{\partial f_{12}}{\partial x_{l_{12}}}=\frac{\partial f_{12}}{\partial x_{13}}=\frac{\partial f_{12}}{\partial x_{14}}=0, \frac{\partial f_{12}}{\partial x_{l_{6}}}=-x_{12} \frac{\partial \mu}{\partial x_{l_{6}}}, \frac{\partial f_{12}}{\partial x_{11}}=u_{41}, \\
& \frac{\partial f_{12}}{\partial x_{12}}=-\left(u_{42}+\mu\right), \frac{\partial f_{13}}{\partial x_{1}}=\frac{\partial f_{13}}{\partial x_{3}}=\frac{\partial f_{13}}{\partial x_{6}}=\frac{\partial f_{13}}{\partial x_{7}}=\frac{\partial f_{13}}{\partial x_{l_{13}}}=\frac{\partial f_{13}}{\partial x_{14}}=0, \frac{\partial f_{13}}{\partial x_{l_{6}}}=-x_{13} \frac{\partial \mu}{\partial x_{l_{6}}}, \\
& \frac{\partial f_{13}}{\partial x_{13}}=-\left(u_{43}+\mu\right), \frac{\partial f_{14}}{\partial x_{1}}=\frac{\partial f_{14}}{\partial x_{l_{3}}}=\frac{\partial f_{14}}{\partial x_{l_{14}}}=0, \frac{\partial f_{14}}{\partial x_{l_{6}}}=-x_{14} \frac{\partial \mu}{\partial x_{l_{6}}}, \frac{\partial f_{14}}{\partial x_{14}}=u_{44}-u_{45}-\mu \text {. }
\end{aligned}
$$

\section{References}

[1] D. Dietz, A.P. Zeng, Efficient production of 1,3-propanediol from fermentation of crude glycerol with mixed cultures in a simple medium, Bioprocess Biosyst. Eng. 37(2014)225-233.

[2] M. Metsoviti, K. Paraskevaidi, A. Koutinas, A.P. Zeng, S. Papanikolaou, Production of 1,3-propanediol, 2,3-butanediol and ethanol by a newly isolated Klebsiella oxytoca strain growing on biodiesel-derived glycerol based media, Process Biochem. 47(12)(2012)1872-1882.

[3] C.E. Nakamura, G.M. Whited, Metabolic engineering for the microbial production of 1,3-propanediol, Curr. Opin. Biotechnol. 14(15)(2003)454-459.

[4] M. Metsoviti, A.P. Zeng, A. Koutinas, K. Paraskevaidi, Enhanced 1,3-propanediiol production by a newly isolated Citrobacter freundii strain cultivated on biodiesel-derived waste glycerol through sterile and non-sterile bioprocesses, J. Biotechnol. 163(2013)408-418.

[5] A.P. Zeng, H. Biebl, Bulk chemicals from biotechnology: the case of 1,3-propanediol production and the new trends, Adv. Biochem. Eng. Biotechnol. 74(2002)239-259.

[6] A. Ashoori, B. Moshiri, A. Khaki-Sedigh, M. R. Bakhtiari, Optimal control of a nonlinear fed-batch fermentation process using model predictive approach, J. Process Contr. 19(7)(2009)1162-1173.

[7] L. Wang, Q. Lin, R. Loxton, K.L. Teo, G. Cheng, Optimal 1, 3-propanediol production: Exploring the trade-off between process yield and feeding rate variation, J. Process Contr. 32(2015)1-9.

[8] R.K. Saxena, P. Anand, S. Saran, J. Isar, Microbial production of 1,3-propanediol: Recent developments and emerging opportunities, Biotechnol. Adv. 27 (2009) 895-913.

[9] A.P. Zeng, A kinetic model for product formation of microbial and mammalian cells, Biotechnol. Bioeng. 46(4)(1995)314-324. 
[10] Y.Q. Sun, W.T. Qi, H. Teng, Z.L. Xiu, A.P. Zeng, Mathematica modeling of glycerol fermentation by Klebsiella pneumoniae: Concern ing enzyme-catalytic reductive pathway and transport of glycerol and 1,3-propanediol across cell membrane, Biochem. Eng. J. 38(2008)22-32.

[11] J. Shao, X. Zhang, E. Feng, Z. Xiu, Experimental design suboptimization for the enzyme-catalyticnonlinear time-delay system in microbial batch culture, J. Process Contr. 24(2014)1740-1746.

[12] G.M. Cheng, L. Wang, R.C. Loxton, Q. Lin, Robust optimal control of a microbial batch culture process, J. Optim. Theory. Appl. (2014), http://dx.doi.org/10.1007/s10957-014-0654-Z.

[13] Z.G. Jiang, J.L. Yuan, E.M. Feng, Robust Identication and Its Properties of Nonlinear Bilevel Multi-Stage Dynamic System, Appl. Math. Comput. 219(12)(2013)6979-6985.

[14] H. Yin, J.L. Yuan, X. Zhang, J. Xie, E. Feng, Z. Xiu, Modeling and parameter identification for a nonlinear multi-stage system for dha regulon in batch culture, Appl. Math. Modell. (2015), http://dx.doi.org/10.1016/j.apm.2015.04.051

[15] J.L. Yuan, X. Zhang, X. Zhu, H.C. Yin, E.M. Feng, Z.L. Xiu, B. Tan, Identification and robustness analysis of nonlinear multi-stage enzymecatalytic dynamical system in batch culture, Comp. Appl. Math. 34(2015)957-978.

[16] J. Wang, J.X. Ye, H.C. Yin, E.M. Feng, L. Wang, Sensitivity analysis and identification of kinetic parameters in batch fermentation of glycerol, J. Comput. Appl. Math. 236(9)(2012)2268-2276.

[17] J.L. Yuan, X. Zhu, X. Zhang, H.C. Yin, E.M. Feng, Z.L. Xiu, Robust identification of enzymatic nonlinear dynamical systems for 1,3propanediol transport mechanisms in microbial batch culture, Appl. Math. Comput. 232(2014)150-163.

[18] J.L. Yuan, X. Zhang, X. Zhu, E.M. Feng, H.C. Yin, Z.L. Xiu, Modelling and pathway identification involving the transport mechanism of a complex metabolic system in batch culture, Commun. Nonlinear Sci. Numer. Simulat. 19(16)(2014)2088-2103.

[19] Y. Wang, L. Wang, Y. Zhao, A. Song, Y. Ma, A stochastic model for microbial fermentation process under Gaussian white noise environment, Numer. Algebra Control Optim. 5(4)(2015)381-392.

[20] X. Zhu, E.M. Feng, Joint estimation in batch culture by using unscented kalman filter, Biotechnol. Bioproc. Engineering. 17(6)(2012)12381243.

[21] J. Wang, J. Ye, E.M. Feng, H. Yin, Z. Xiu, Modeling and identification of a nonlinear hybrid dynamical system in batch fermentation of glycerol, Math. Comput. Model. 54(2011)618-624.

[22] I.T. Tong, H.H. Liao, D.C. Cameron, 1,3-Propanediol production by Escherichia coli expressing genes from the Klebsiella pneumoniae dha regulon, Appl. Environ. Microb. 57 (12)(1991)3541-3546.

[23] J. Sun, J. Heuvel, P. Soucaille, Y. Qu, A.P. Zeng, Comparative genomic analysis of dha regulon and related genes for anaerobic gelycerol metabolism in bacteria, Biotechnol. Progr. 19(2003)263-272.

[24] I.T. Tong, D.C. Cameron, Enhancement of 1,3-propanediol production by cofermentation in Escherichia coli expressing Klebsiella pneumoniae dha regulon genes, Appl. Biochem. Biotech. 34-35(1992)149-159.

[25] Y.Q. Sun, J.X. Ye, X.J. Mu, H. Teng, E.M. Feng, Z.L. Xiu, A.P. Zeng, Nonlinear mathematical simulation and analysis of dha regulon for glycerol metabolism in klebsiella pneumoniae, Chinese J. Chem. Eng. 20(5)(2012)958-970.

[26] J.L. Yuan, X. Zhang, X. Zhu, E. Feng, H. Yin, Z. Xiu, Pathway identification using parallel optimization for a nonlinear hybrid system in batch culture, Nonlinear Analysis: Hybrid Sys. 15(2015)112-131.

[27] G.J. Silva, A. Datta, S.P. Bhattacharyya, PID controllers for time-delay systems, Springer, 2007

[28] L. Denis-Vidal, C. Jauberthie, G. Joly-Blanchard, Identifiability of a nonlinear delayed-differential aerospace model, IEEE T. Automat. Contr. 51(2006)154-158.

[29] P. Yan, H. Ozbay, Stability analysis of switched time delay systems, SIAM J. Control Optim. 47(2)(2008) 936-949.

[30] M. Anguelova, B. Wennberg, State elimination and identifiability of the delay parameter for nonlinear time-delay systems. Automatica 44(2008)1373-1378.

[31] Q.Q. Chai, R. Loxton, K.L. Teo, C. Yang, A class of optimal state-delay control problems, Nonlinear Anal. Real. 14(2013)1536-1550.

[32] Q.Q. Chai, R. Loxton, K.L. Teo, C. Yang, A unified parameter identification method for nonlinear time-delay systems. J. Ind. Manag. Optim. 9(2)(2013)471-486

[33] Q.Q. Chai, R. Loxton, K.L. Teo, C. Yang, Time-delay estimation for nonlinear systems with piecewise-constant input. Appl. Math. Comput. 219(17)(2013)9543-9560.

[34] C.Y. Liu, R. Loxton, K.L. Teo, Switching time and parameter optimization in nonlinear switched systems with multiple time-delays. J. Optim. Theory Appl. 163(3)(2014)957-988.

[35] Z.G. Wu, J.H. Park, H. Su, J. Chu, Stochastic stability analysis of piecewise homogeneous markovian jump neural networks with mixed time-delays, J. Franklin I. 349 (2012) 2136-2150.

[36] Z.G. Wu, J.H. Park, H. Su, J. Chu, Passivity analysis of markov jump neural networks with mixed time-delays and piecewise-constant transition rates, Nonlinear Anal. Real 13(2012)2423-2431.

[37] MacDonald N.: Time-lags in Biological Models, Lecture Notes in Biomathematics, 27, Springer, Berlin, 1979.

[38] Z.G. Wu, J.H. Park, H. Su, J. Chu, Stochastic stability analysis for discrete-time singular markov jump systems with time-varying delay and piecewiseconstant transition probabilities, J. Franklin I. 349 (2012) 2889-2902.

[39] C.Y. Liu, R. Loxton, K.L. Teo, Optimal parameter selection for nonlinear multistage systems with time-delays, Comput. Optim. Appl. 59(12)(2014)285-306.

[40] R. Loxton, K.L. Teo, R. Volker, An optimization approach to state-delay identification, IEEE T. Automat. Contr. 55(2010) $2113-2119$.

[41] H. Kitano, Biological robustness, Nat. Rev. Genet. 5(11)(2004)826-837.

[42] M. Perc, M. Marhl, Sensitivity and flexibility of regular and chaotic calcium oscillations, Biophys. Chem. 104(2)(2003)509-522.

[43] M. Perc, M. Marhl, Noise enhances robustness of intracellular Ca ${ }^{2+}$ oscillations, Phys. Lett. A 316(5)(2003)304-310.

[44] J. Stelling, U. Sauer, Z. Szallasi, F.J. Doyle, J. Doyle, Robustness of cellular functions, Cell 118(6)(2004)675-685.

[45] H. Kitano, Towards a theory of biological robustness, Mol. Syst. Biol. 3(2007)(Article ID 137).

[46] N. Barkai, S. Leibler, Robustness in simple biochemical networks, Nature 387(6636)(1997)913-917.

[47] V. Rehbock, K.L. Teo, L.S. Jennings, A computational procedure for suboptimal robust controls, Dynam. Control 2(1992)331-348.

[48] W. Wei, K.L. Teo, Z. Zhan, A numerical method for an optimal control problem with minimum sensitivity on coefficient variation, Appl. 
Math. Comput. 218(2011)1180-1190.

[49] R.C. Loxton, K.L. Teo, V. Rehbock, Robust suboptimal control of nonlinear systems, Appl. Math. Comput. 217(2011)6566-6576.

[50] K. Malanowski, Sensitivity analysis for parametric optimal control of semilinear parabolic equations. J. Convex Analy. 9(2002)543-561.

[51] J.L. Yuan, L. Wang, X. Zhang, E. Feng, H. Yin, Z. Xiu, Parameter identification for a nonlinear enzyme-catalytic dynamic system with time-delays, J. Glob. Optim. 62(2015)791-810.

[52] L. Wang, Determining the transport mechanism of an enzyme-catalytic complex metabolic network based on biological robustness, Bioproc. biosyst. eng. 36(4)(2013)433-441.

[53] K.L. Teo, C.J. Goh, K.H. Wong, A unified computational approach to optimal control problems, Long Scientific Technical, Essex 1991.

[54] E. Polak, Optimization Algorithms and Consistent Approximations. Springer-Verlag, New York 1997.

[55] R.P. Ge, A filled function method for finding a global minimizer of a function of several variables. Math. Program. 46(1990)191-204.

[56] F. Wei, Y. Wang, H. Lin, A new filled function method with two parameters for global optimization, J. Optim. Theory Appl. 163(2014)510527.

[57] H. Lin, Y. Gao, Y. Wang, A continuously differentiable filled function method for global optimization, Numer. Algor. 66(2014)511-523.

[58] P. Michaels, B. Zubik-Kowal, Parallel computations and numerical simulations for nonlinear systems of Volterra integro-differential equations, Commun. Nonlinear Sci. Numer. Simulat. 17(7)(2012)3022-3030.

[59] A. Ellermeyer, J. Hendrix, N. Ghoochan, A theoretical and empirical investigation of delayed growth response in the continuous culture of bacteria, J. Theor. Biol. 222(2003)485-494.

[60] R. Sharma, Some more inequalities for arithmetic mean, harmonic mean and variance, J. Math. Inequalities, 2(1)(2008)109-114.

[61] J. Nocedal, S.J. Wright, Numerical optimization, Springer-Verlag, New York, 1999.

[62] F.H. Clarke, Yu.S. Ledyaev, R.J. Stem, P.R. Wolenski, Nonsmooth Analysis and Control Theory. Graduate Texts in Mathematics, vol.178. Springer-Verlag, New York, 1998. 